\title{
ON THE EXISTENCE OF CONVEX CLASSICAL SOLUTIONS FOR MULTILAYER FREE BOUNDARY PROBLEMS WITH GENERAL NONLINEAR JOINING CONDITIONS
}

\author{
ANDREW ACKER
}

\begin{abstract}
We prove the existence of convex classical solutions for a general multidimensional, multilayer free-boundary problem. The geometric context of this problem is a nested family of closed, convex surfaces. Except for the innermost and outermost surfaces, which are given, these surfaces are interpreted as unknown layer-interfaces, where the layers are the bounded annular domains between them. Each unknown interface is characterized by a quite general nonlinear equation, called a joining condition, which relates the first derivatives (along the interface) of the capacitary potentials in the two adjoining layers, as well as the spatial variables. A well-known special case of this problem involves several stationary, immiscible, two-dimensional flows of ideal fluid, related along their interfaces by Bernoulli's law.
\end{abstract}

\section{INTRODUCTION}

Our purpose is to study the existence question for convex classical solutions of multilayer free boundary problems of the following general form:

1.1. Problem. In $\mathbb{R}^{N}, N \geq 2$, let an annular domain $\Omega_{*}$ of the form $\Omega_{*}$ $=D_{*}^{+} \backslash C l\left(D_{*}^{-}\right)$be given, where $C l(\cdot)$ denotes the closure of a set. Here, $D_{*}^{ \pm}$ denote fixed, bounded, convex, nested domains with boundaries $S_{*}^{ \pm}=\partial D_{*}^{ \pm}$. Given the twice continuously-differentiable functions $F_{i}(x, p, q): \mathbb{R}^{N} \times \mathbb{R}_{+} \times \mathbb{R}_{+} \rightarrow \mathbb{R}$, $i=1, \ldots, k$, we seek an ordered family of convex domains $\left(D_{1}, \ldots, D_{k}\right)$ such that $C l\left(D_{i}\right) \subset D_{i+1}$ for $i=0,1, \ldots, k$ (where $D_{0}=D_{*}^{-}$and $D_{k+1}=D_{*}^{+}$), and such that

$$
F_{i}\left(x,\left|\nabla U_{i}(x)\right|,\left|\nabla U_{i+1}(x)\right|\right)=0 \text { on } S_{i}:=\partial D_{i}
$$

for $i=1, \ldots, k$, where $U(x)$ solves the boundary value problem

$$
\Delta U(x)=0 \text { in } \Omega_{*} \backslash\left(S_{1} \cup \cdots \cup S_{k}\right), \quad U\left(S_{i}\right)=i(i=0, \ldots, k, k+1),
$$

and where $U_{i}$ denotes the restriction of $U$ to the (closure of the) annular domain $\Omega_{i}:=D_{i} \backslash C l\left(D_{i-1}\right)$.

In Problem 1.1, equation (1.1) represents a general, nonlinear "joining condition" relating the first derivatives of the capacitary potentials on opposite sides of each of the (convex) free-boundary surfaces $S_{i}, i=1, \ldots, k$. The main focus of this paper

Received by the editors August 15, 1995.

1991 Mathematics Subject Classification. Primary 35R35, 35J05, 76T05.

Key words and phrases. Multilayer elliptic free boundary problem, convexity, non-linear joining conditions. 
is on obtaining existence results for Problem 1.1 which apply to a very general class of nonlinear joining conditions.

The author's previous existence results for Problem 1.1 (see [7], [8], [9]) are limited to joining conditions of the form:

$$
\left|\nabla U_{i}(x)\right|^{2}=\left|\nabla U_{u+1}(x)\right|^{2}+A_{i}(x) \text { on } S_{i},
$$

$i=1, \ldots, k$, corresponding to functions $F_{i}(x, p, q)=A_{i}(x)+q^{2}-p^{2}$ in which $A_{i}(x)>0$ and the related functions $\left(1 / \sqrt{A}_{i}(x)\right)$ are concave in $\Omega_{*}$. To the author's knowledge, the only alternative treatment of the existence question for the convex multilayer problem is due to Laurence and Stredulinsky [20], [21]. Their existence results in [21] are limited to joining conditions of the form (1.3) in the particular case where $N=2$ and each function $A_{i}(x)$ is a positive constant. (No existence proof is yet available for the interesting and closely related multilayer free-boundary problem proposed in [20]. See [6].) The most general available existence results for the two-layer problem in the geometrically general (non-convex) setting, due to Alt, Caffarelli, and Friedman [13], also involve joining conditions of the form (1.3), in which, however, the function $A_{1}(x)$ satisfies no requirements beyond regularity. The existence proof of Alt, Caffarelli, and Friedman is based on the method of variational inequalities, and the convex existence proofs in [9] and [21] were accomplished essentially by adapting the method of variational inequalities to a convex setting (in the tradition of the convex variational proofs in [2], [3], [15]). We remark that the joining condition (1.3) is of special interest in the context of the theory of two-dimensional ideal fluids, where Problem 1.1 can be interpreted in terms of a layered flow involving several immiscible ideal fluids. In this setting, (1.3) can be interpreted as a relation between the speeds of the neighboring flows along their interface, governed by Bernoulli's law of fluids.

Our present existence results will be stated in $\S 2$ (see Theorems 2.3 and 2.11), along with a number of concrete special cases which very substantially generalize the form given in (1.3). The main obstacle to the proof of these results is the absence of any variational formulation for the generalized problem, necessitating a new proof based on an alternative principle. Essentially, the new proof is an adaptation of the idea of obtaining a solution as the limit of an increasing (resp. decreasing) sequence of inner (outer) solutions (see $\$ \S 3$ and 5).

The author's old and new existence results for Problem 1.1 are all based on the application of a one-parameter family of free-boundary perturbation operators $T_{\varepsilon}, 0<\varepsilon<1$, which preserve the geometric convexity of the members of the ordered families of free boundaries occurring in the problem. The author originally introduced these operators in [1], adapted them to convex free boundary problems in [2] and [3], and then adapted them (together with their various properties and uses) to the case of joining conditions of the form (1.3) in [5], [7], [8] and [9]. In [9], the operators $T_{\varepsilon}, 0<\varepsilon<1$, are used to define the family of convexity-preserving variations of the free boundaries by which it is shown that the convex functional minimizer satisfies (1.3) in a weak sense. For a general discussion of the variational aspects of the operator method in the context of a convex free-boundary problems, we refer the reader to the author's survey article [10]. In essence, the present generalization of the existence results in [7], [8], and [9] is accomplished by applying the same operators $T_{\varepsilon}$, but in generalized form corresponding to the generalized functions $F_{i}(x, p, q), i=1, \ldots, k$ (see [11]), and in a new context in which the previous emphasis on their variational properties is replaced by an emphasis on 
their monotonicity properties. By means of these operators, we define (for each $0<\varepsilon<1$ ) a multilayer fixed-point problem which approximates Problem 1.1 (for small $\varepsilon$ ). By using the monotonicity properties of the operators $T_{\varepsilon}$, we prove that for each sufficiently small $\varepsilon>0$, this multilayer fixed-point problem has a solution which is obtained as the limit of an increasing (or decreasing) sequence of successive approximations which are all inner (outer) solutions (see $\S \S 3-5$ ). Then the "weak" solutions of Problem 1.1 are defined to be the limits of convergent sequences of these multilayer fixed points (corresponding to positive null sequences of values of $\varepsilon$ ). We then show (in $§ \S 6-10$ ) that the weak solutions of Problem 1.1 are actually classical solutions.

It is the author's impression of the literature on free boundary problems (of all kinds) that the conditions characterizing the free boundaries are usually very specific (in each particular problem). By comparison, the present paper studies the existence question for an unusually broad class of joining conditions (see §2). However, it is important to realize that the convexity requirement in Problem 1.1 necessitates restrictions on the joining conditions (1.1) which go well beyond the regularity of the functions $F_{i}(x, p, q), i=1, \ldots, k$, and the solvability of the equations $F_{i}(x, p, q)=0$ for either $p$ or $q$ in terms of the other variables. In fact the author showed by a counterexample in [6] that (in general) no convex solution exists even in the two-dimensional, two-layer case of Problem 1.1 if the one joining condition is of the form (1.3), where $A(x)$ is a negative constant.

For the purpose of focusing attention more directly on the quite difficult questions associated with the treatment of very general, nonlinear joining conditions of the form (1.1), the present treatment of the multilayer problem has been restricted to the case where $U$ is harmonic in the layers $\Omega_{i}$ between the free boundaries. However, the author is quite certain that the operator structure explored here can be generalized to other cases, in which $U$ solves various other elliptic partial differential equations in the layers between the free boundaries (see [11], [12], [23] regarding convergence properties of the operators $T_{\varepsilon}$ in the case of one-layer and two-layer problems for the $p$-Laplacian). In this context, it should be apparent that the author's primary motive for the present work is to develop an alternative, non-variational version of the general operator method with potential application to numerous (convex) multilayer problems with general joining conditions. The author also hopes to extend the operator method to obtain existence results for Problem 1.1 in the corresponding starlike case.

1.2. Remark. A powerful simplifying principle for the multilayer problem is the obvious fact that if $S=\left(S_{1}, \ldots, S_{k}\right)$ is a classical (or weak) solution of Problem 1.1 , then for each $i=1, \ldots, k$, the surface $S_{i}$ is a classical (or weak) solution of a two-layer version of Problem 1.1 (see Problem 2.1) relative to its neighboring surfaces $S_{i \pm 1}$. This principle permitted the author to simplify the treatment of the convex variational multilayer problems in [7], [8], [9] by a reduction of the most difficult aspects of the problem to a suitable analysis of the two-layer case. Although the present paper also makes maximum use of the same principle (see $\S \S 2-5)$, it is no longer possible to avoid directly studying the operators $T_{\varepsilon}$ in the multilayer context.

1.3. Remark. Although the method of variational inequalities has been applied very successfully in the study of numerous free boundary problems, there is great aesthetic appeal in the idea of extending the study of the multilayer problem beyond 
the scope of this method. This endeavor also has much practical significance, since there are numerous interesting joining conditions of the form (1.1) which do not appear to arise from any natural variational formulation of the problem, but which might nevertheless occur in models of observed physical phenomena. A number of such examples are given in $\S \S 2.6-2.9$.

1.4. Remark. An important aspect of the operator method in all cases is the fact that each existence proof embodies (in analytical form) an algorithm for the successive approximation of solutions. The aspect of successive approximation was particularly emphasized in [4] and [5].

\section{MAin Results}

It is natural to begin any discussion of Problem 1.1 with the important particular case of two layers and one free boundary. Our study of the general problem is in many ways based on a reduction to this particular case.

2.1. Two-layer problem. In $\mathbb{R}^{N}, N \geq 2$, let an annular domain $\Omega_{*}$ be given of the form $\Omega_{*}=D_{*}^{+} \backslash C l\left(D_{*}^{-}\right)$. Here, $D_{*}^{ \pm}$denote fixed, bounded, convex, nested domains with boundaries $S_{*}^{ \pm}=\partial D_{*}^{ \pm}$. Given a twice continuously-differentiable function $F(x, p, q): \mathbb{R}^{N} \times \mathbb{R}_{+} \times \mathbb{R}_{+} \rightarrow \mathbb{R}$, we seek a convex domain $D$ such that $C l\left(D_{*}^{-}\right) \subset D \subset C l(D) \subset D_{*}^{+}$, and

$$
F\left(x,\left|\nabla U^{-}(x)\right|,\left|\nabla U^{+}(x)\right|\right)=0 \text { on } S:=\partial D,
$$

where the functions $U^{ \pm}(x)$ solve the boundary value problems

$$
\Delta U^{ \pm}(x)=0 \text { in } \Omega^{ \pm}, \quad U^{ \pm}(S)=0, U^{ \pm}\left(S_{*}^{ \pm}\right)=1,
$$

and where $\Omega^{ \pm}$denotes the annular domain whose boundary is given by $\partial \Omega^{ \pm}=$ $S \cup S_{*}^{ \pm}$.

2.2. Assumptions for Problem 2.1. Define the twice-continuously-differentiable function $f(x, p, q): \mathbb{R}^{N} \times \mathbb{R}_{+} \times \mathbb{R}_{+} \rightarrow \mathbb{R}$ such that $f(x, p, q)=F(x, 1 / p, 1 / q)$. Throughout this paper, our results in the context of Problem 2.1 will all require various combinations of (but not necessarily all of) the following additional assumptions:

(a) The function $F(x, p, q)$ is strictly decreasing in $p$ and strictly increasing in $q$. Thus $f(x, p, q)$ is strictly increasing in $p$ and strictly decreasing in $q$.

(b) For any $p_{0}>0$, there exists a value $q_{0}=q_{0}\left(p_{0}\right)>0$ such that $F\left(x, p_{0}, q\right)>0$ whenever $q>q_{0}$, independent of $x \in \mathbb{R}^{N}$. For any $q_{0}>0$, there exist values $0<p_{0}=p_{0}\left(q_{0}\right)<p_{1}=p_{1}\left(q_{0}\right)$ such that $F\left(x, p, q_{0}\right)>0$ whenever $0<p<p_{0}$ and $F\left(x, p, q_{0}\right)<0$ whenever $p>p_{1}$, independent of $x \in \mathbb{R}^{N}$.

(c) For any $r_{0}>0$, there exists a value $\theta_{0}=\theta_{0}\left(r_{0}\right)>0$ such that

$$
f\left(x, r_{0} \cos (\theta), r_{0} \sin (\theta)\right)>0
$$

for all $x \in \mathbb{R}^{N}$ and $\theta \in\left(0, \theta_{0}\right]$, whereas $f(x, r \cos (\theta), r \sin (\theta))<0$ for all $x \in \mathbb{R}^{N}, \theta \in$ $\left[(\pi / 2)-\theta_{0},(\pi / 2)\right)$, and $r \in\left(0, r_{0}\right]$.

(d) For any linear function $\phi(\lambda):[0,1] \rightarrow \Omega_{*} \times \mathbb{R}_{+} \times \mathbb{R}_{+}$such that $f(\phi(0)) \leq 0$ and $f(\phi(1)) \leq 0$, we have that $\max \{f(\phi(\lambda)): 0 \leq \lambda \leq 1\} \leq 0$.

(e) We assume $F(x, p, q)=F^{+}(x, q)-F^{-}(x, p)$ for all $(x, p, q) \in \mathbb{R}^{N} \times \mathbb{R}_{+} \times \mathbb{R}_{+}$, where the positive functions $F^{ \pm}(x, t): \mathbb{R}^{N} \times \mathbb{R}_{+} \rightarrow \mathbb{R}$ are both twice continuously differentiable, and are such that $\partial F^{ \pm}(x, t) / \partial t>0$ and $\partial^{2} F^{-}(x, t) / \partial t^{2} \geq 0$. 
2.3. Theorem. (a) Problem 2.1 has at least one weak solution $\widetilde{S}$ (in the sense of Definition 3.12), provided that the given function $F(x, p, q)$ satisfies Assumptions $2.2(\mathrm{a})-(\mathrm{d})$.

(b) Under Assumptions 2.2(a)-(e), any weak solution $\widetilde{S}$ of Problem 2.1 is a classical solution.

2.4. Remark. (a) The proof of Theorem 2.3(a) is given in $\S 3.17$, while the proof of Part (b) is given in Theorem 10.1

2.5. Remarks. (a) Under Assumption 2.2(a), let the equation $f(x, p, q)=0$ be solved by $p=h(x, q): \mathbb{R}^{N} \times \mathbb{R}_{+} \rightarrow \mathbb{R}_{+}$. Then Assumption $2.2(\mathrm{~d})$ is satisfied if, for any linear mapping $\phi(\lambda):[0,1] \rightarrow \Omega_{*}$, the function $\psi(\lambda, q):=h(\phi(\lambda), q)$ satisfies $\psi_{\lambda \lambda} \leq 0, \psi_{q q} \leq 0$, and $\psi_{\lambda q}^{2} \leq \psi_{\lambda \lambda} \psi_{q q}$, where the subscripts denote partial differentiation. (b) In the context of Part (a), let Assumption 2.2(b) hold. Then $h(x, q) \rightarrow 0+$ (uniformly over all $\left.x \in \mathbb{R}^{N}\right)$ as $q \rightarrow 0+$. If $f\left(x, r_{0} \cos (\theta), r_{0} \sin (\theta)\right)<0$ for fixed $x \in \mathbb{R}^{N}, \theta \in(0, \pi / 2)$, and $r_{0}>0$, then $h\left(x, r_{0} \sin (\theta)\right)>r_{0} \cos (\theta)$. If one assumes that $h_{q q} \leq 0$ (as in Part (a)), then it follows that $h(x, r \sin (\theta))>r \cos (\theta)$ for all $r \in\left(0, r_{0}\right]$, whence $f(x, r \cos (\theta), r \sin (\theta))<0$ for all $r \in\left(0, r_{0}\right]$.

2.6. Example. Theorem 2.3 applies in the case of numerous nonlinear joining conditions (2.1) in which $F(x, p, q)$ does not depend on position. Essentially all such cases can be expressed in the form $F(x, p, q):=\phi(q)-p=0$. Then Assumptions 2.2(a)-(e) are all satisfied if the equation $F(x, p, q)=0$ is equivalent to an equation of the form $p h(1 / q)=1$, where $h(q): \mathbb{R}_{+} \rightarrow \mathbb{R}_{+}$denotes any $C^{2}$-function such that $h_{q}>0, h_{q q} \leq 0$, and $h(q) \rightarrow 0+$ as $q \rightarrow 0+($ clearly $\phi(q)=1 / h(1 / q))$. For example, Assumptions 2.2(a)-(e) are all satisfied by joining conditions of the following forms: $p=q^{\alpha}$ for fixed $\alpha \in(0,1] ; p \sum_{i=1}^{n} a_{i} q^{-\alpha_{i}}=1$, where $a_{i}>0$ and $0<\alpha_{i} \leq 1$; $q(\cosh (1 / p)-1)=1 ; q \tan (1 / p)=1 ; p \ln (1+(1 / q))=1 ; q \sinh (1 / p)=1$.

2.7. Example. It is easily seen (using Remarks 2.5) that Assumptions 2.2(a)-(e) are all satisfied by joining equations of the form $F(x, p, q):=A(x)+\phi(1 / q)-p=0$, where $A(x): \mathbb{R}^{N} \rightarrow \mathbb{R}$ is a uniformly positive $C^{2}$-function such that the first and second order directional derivatives of $A(x)$ satisfy the condition: $2 A_{\nu}^{2} \leq A A_{\nu \nu}$ throughout $\Omega_{*}$ for any unit vector $\nu$, and where $\phi(q): \mathbb{R}_{+} \rightarrow \mathbb{R}_{+}$is a positive $C^{2}$ function such that $\phi(q) \rightarrow \infty$ as $q \rightarrow 0+, \phi_{q}<0$, and $2 \phi_{q}^{2} \leq \phi \phi_{q q}$. (This includes the case where $\phi(q)=q^{-\alpha}$ for some fixed $\alpha>0$.) Therefore, Theorem 2.3 applies to these cases.

2.8. Example. It is easily seen (using Remarks 2.5) that Assumptions 2.2(a)-(e) are all satisfied by joining equations of the form $A(x) \phi(1 / q) p=1$ (corresponding to $F(x, p, q):=(1 /[A(x) \phi(1 / q)])-p=0)$, where the $C^{2}$-functions $A(x): \mathbb{R}^{N} \rightarrow \mathbb{R}$ and $\phi(q): \mathbb{R}_{+} \rightarrow \mathbb{R}_{+}$are chosen such that $A(x)$ is uniformly positive, $\phi_{q}>0, \phi(q) \rightarrow 0$ as $q \rightarrow 0+$, and there exist fixed positive constants $\lambda, \mu$ with $\lambda \mu=1$ such that $\phi \phi_{q q}+\mu \phi_{q}^{2} \leq 0$ for all $q>0$ and $A A_{\nu \nu}+\lambda A_{\nu}^{2} \leq 0$ for all $x \in \Omega_{*}$ and all unit vectors $\nu$. Therefore, Theorem 2.3 applies to these cases.

2.9. Example. Consider joining conditions of the general form:

$$
F(x, p, q)=A(x)+B(x) q^{\beta}-p^{\alpha}=0,
$$

where $\alpha, \beta$ denote positive constants and $A(x), B(x)$ denote uniformly-positive $C^{2}$ functions. Assumptions 2.2(a), (b), (c) are clearly satisfied. By expressing the joining condition in the equivalent form: $F_{0}(x, p, q):=\left(A+B q^{\beta}\right)^{(1 / \alpha)}-p=0$, one 
can easily verify that Assumptions 2.2(e) is also satisfied. As we will show in the appendix ( $(11)$, Assumption 2.2(d) is satisfied provided that $\beta<\alpha$ and the first and second order directional derivatives of $A(x)$ and $B(x)$ satisfy the conditions:

$$
((\alpha+1) / \alpha) A_{\nu}^{2} \leq A A_{\nu \nu} ; \quad(1+(1 /(\alpha-\beta))) B_{\nu}^{2} \leq B B_{\nu \nu}
$$

in $\Omega_{*}$, both for any unit vector $\nu$. (Alternately, one can assume that $\alpha=\beta, B(x)$ is a constant, and $((\alpha+1) / \alpha) A_{\nu}^{2} \leq A A_{\nu \nu}$ in $\Omega_{*}$ for each direction $\nu$.) Thus, Theorem 2.3 applies under these assumptions. The convex existence results in this example are very general by contrast to the author's previous existence results for the convex two-layer problem (see [9]).

2.10. Remark. With reference to the author's counterexamples in [6], we remark that the function $F(x, p, q):=\lambda+q^{2}-p^{2}$ satisfies Assumptions 2.2(a)-(e) in the case where $\lambda>0$, but does not satisfy Assumption 2.2(d) in the case where $\lambda<0$, for which the counterexamples were obtained.

2.11. Theorem. (a) Problem 1.1 has at least one (convex) weak solution $\widetilde{S}=$ $\left(\widetilde{S}_{1}, \ldots, \widetilde{S}_{k}\right)$ (weak in the sense of Definition 5.4 ), provided that each of the given functions $F_{i}(x, p, q): \mathbb{R}^{n} \times \mathbb{R}_{+} \times \mathbb{R}_{+} \rightarrow \mathbb{R}, i=1, \ldots, k$, satisfies Assumptions $2.2(\mathrm{a})-(\mathrm{d})$.

(b) Any weak solution $\widetilde{S}=\left(\widetilde{S}_{1}, \ldots, \widetilde{S}_{k}\right)$ of Problem 1.1 is a classical solution, provided that each of the given functions $F_{i}(x, p, q): \mathbb{R}^{N} \times \mathbb{R}_{+} \times \mathbb{R}_{+} \rightarrow \mathbb{R}, i=$ $1, \ldots, k$, satisfies Assumptions 2.2(a)-(e).

2.12. Remark. The proof of Theorem 2.11(a) is given in $\$ 5.12$. Part (b) follows from Theorem 2.3(b), in view of Theorem 5.13.

2.13. Remark. Obviously Theorem 2.11 applies to particular cases of Problem 1.1 in which each of the functions $F_{i}(x, p, q), i=1, \ldots, k$, has any one of the forms already discussed in Examples 2.6, 2.7, 2.8, and 2.9.

2.14. Remark. The purpose of this remark is to point out a uniqueness result which applies to classical solutions of Problem 1.1 under different assumptions than those listed in $§ 2.2$. Namely, assume in Problem 1.1 that the given domains $D_{*}^{ \pm}$are starlike relative to the origin, and that the functions $F_{i}(x, p, q), i=1, \ldots, k$, satisfy Assumption 2.2(a). Also assume for $i=1, \ldots, k$ that $F_{i}(\lambda x, p / \lambda, q / \lambda) \geq 0$ whenever $F_{i}(x, p, q) \geq 0$ and $\lambda \geq 1$. Then there exists at most one classical solution $\widetilde{S}=$ $\left(\widetilde{S}_{1}, \ldots, \widetilde{S}_{k}\right)$ such that the surfaces $\widetilde{S}_{i}, i=1, \ldots, k$, all have bounded curvature. Moreover, this solution (if it exists) must be such that the interior complements of the surfaces $\widetilde{S}_{1}, \ldots, \widetilde{S}_{k}$ are all starlike. The proof, which we omit, is closely related to previous uniqueness proofs in [7], [8], $§ 2$, and [11], $§ 2$.

\section{THE CONVEX TWO-LAYER PROBLEM: OPERATORS, FIXED POINTS, WEAK SOLUTIONS}

3.1. General notation and definitions. We define the ball $B_{\varepsilon}\left(x_{0}\right)=B\left(x_{0} ; \varepsilon\right)=$ $\left\{x \in \mathbb{R}^{N}:\left|x-x_{0}\right|<\varepsilon\right\}$ for any $x_{0} \in \mathbb{R}^{N}$ and $\varepsilon>0$. We define $\alpha \Sigma=\{\alpha x: x \in \Sigma\}$ for any set $\Sigma$ and value $\alpha>0 . H(\Sigma)$ denotes the convex hull of a set $\Sigma$, and $N_{\varepsilon}(\Sigma)$ denotes the $\varepsilon$-neighborhood of $\Sigma$ (for any $\varepsilon>0$ ). For any sets $\Sigma_{i}, i=1,2$, we define the distances $d\left(\Sigma_{1}, \Sigma_{2}\right):=\inf \left\{|x-y|: x \in \Sigma_{1}, y \in \Sigma_{2}\right\}$ and $\Delta\left(\Sigma_{1}, \Sigma_{2}\right):=$ $\inf \left\{\varepsilon>0: \Sigma_{1} \subset N_{\varepsilon}\left(\Sigma_{2}\right)\right.$ and $\left.\Sigma_{2} \subset N_{\varepsilon}\left(\Sigma_{1}\right)\right\}$. For a set $\Sigma$ and a point $x_{0}$, we define $d\left(x_{0}, \Sigma\right)=d\left(\left\{x_{0}\right\}, \Sigma\right)$. For any set $\Sigma$ and any sequence of sets $\left(\Sigma_{n}\right)_{n=1}^{\infty}$, we 
say $\Sigma_{n} \rightarrow \Sigma$ as $n \rightarrow \infty$ if $\Delta\left(\Sigma_{n}, \Sigma\right) \rightarrow 0$ as $n \rightarrow \infty$. We use $z(t)$ to denote any particular real valued, continuous, strictly increasing function on $\mathbb{R}_{+}$, such that $z(t) \rightarrow 0$ as $t \rightarrow 0+$, and we use $\zeta(n)$ to denote any particular positive, decreasing function defined on $\mathbb{N}$ such that $\zeta(n) \downarrow 0$ as $n \rightarrow \infty$. Similarly, $z(t, n)$ denotes any particular positive function of $t>0$ and $n \in \mathbb{N}$ such that $z(t, n) \rightarrow 0$ as $t \rightarrow 0$ and $n \rightarrow \infty$. These are all called null functions. Also, $|\cdot|$ denotes volume of an $N$-dimensional domain, the area of an $(N-1)$-dimensional surface, and the length of a curve.

3.2. Definitions for the operator method. Let $\mathbb{X}$ denote the family of all $(N-1)$-dimensional hypersurfaces of the form $S=\partial D$, where $D$ is a bounded, convex domain in $\mathbb{R}^{N}$. For each surface $S \in \mathbb{X}$, let $D(S)$ denote the interior complement for $S$. For $S_{1}, S_{2} \in \mathbb{X}$, we define $S_{1} \leq S_{2}$ (resp. $S_{1}<S_{2}$ ) to mean that $D_{1} \subset D_{2}$ (resp. $\left.C l\left(D_{1}\right) \subset D_{2}\right)$, where $D_{i}=D\left(S_{i}\right), i=1,2$. Given $S_{1}, S_{2} \in \mathbb{X}$ such that $S_{1}<S_{2}$ or $S_{1}>S_{2}$, we use $\Omega\left(S_{1}, S_{2}\right)$ to denote the annular domain whose boundary is $S_{1} \cup S_{2}$. We also use $U^{+}=U^{+}\left(S_{1}, S_{2} ; x\right)$ to denote the capacitary potential in $\Omega=\Omega\left(S_{1}, S_{2}\right)$, oriented such that $U^{+}=0$ (resp. $U^{+}=1$ ) on the inner (outer) boundary of $\Omega$. We also define $U^{-}\left(S_{1}, S_{2} ; x\right)=1-U^{+}\left(S_{1}, S_{2} ; x\right)$. Given $\varepsilon \in(0,1)$ and surfaces $S_{1}, S_{2} \in \mathbb{X}$ with $S_{1}<S_{2}$, we define

$$
\Phi_{\varepsilon}^{ \pm}\left(S_{1}, S_{2}\right)=\Phi^{ \pm}\left(S_{1}, S_{2} ; \varepsilon\right):=\left\{x \in \Omega\left(S_{1}, S_{2}\right): U^{ \pm}\left(S_{1}, S_{2} ; x\right)=\varepsilon\right\} .
$$

Observe that $\Phi_{\varepsilon}^{ \pm}\left(S_{1}, S_{2}\right) \in \mathbb{X}$ due to Lemma 3.4(c). Clearly, we have $S_{1}<$ $\Phi_{\varepsilon}^{ \pm}\left(S_{1}, S_{2}\right)<S_{2}$ and $\Phi_{\varepsilon}^{ \pm}\left(S_{1}, S_{2}\right)=\Phi_{1-\varepsilon}^{\mp}\left(S_{1}, S_{2}\right)$. Also, for $\varepsilon \in(0,1)$ and any surfaces $S_{1}, S_{2} \in \mathbb{X}$ with $S_{1}<S_{2}$, we define

$$
\begin{aligned}
& \Psi_{\varepsilon}\left(S_{1}, S_{2}\right)=\Psi\left(S_{1}, S_{2} ; \varepsilon\right) \\
& \quad:=\partial\left(C l\left(D\left(S_{1}\right)\right) \cup\left\{x \in \Omega\left(S_{1}, S_{2}\right): f\left(x,\left(d\left(x, S_{1}\right) / \varepsilon\right),\left(d\left(x, S_{2}\right) / \varepsilon\right)\right)<0\right\}\right),
\end{aligned}
$$

where $\Psi_{\varepsilon}\left(S_{1}, S_{2}\right) \in \mathbb{X}$ due to Lemma 3.6. Finally, for any $\varepsilon \in(0,1)$ and any surfaces $S, S^{ \pm} \in \mathbb{X}$ with $S^{-}<S<S^{+}$, we define

$$
T_{\varepsilon}\left(S^{-}, S, S^{+}\right)=T\left(S^{-}, S, S^{+} ; \varepsilon\right)=\Psi_{\varepsilon}\left(\Psi_{\varepsilon}^{-}\left(S^{-}, S\right), \Phi_{\varepsilon}^{+}\left(S, S^{+}\right)\right) \in \mathbb{X} .
$$

3.3. Lemma. For surfaces $S_{0}^{ \pm}, S_{1}^{ \pm} \in \mathbb{X}$ such that $S_{0}^{-}<S_{0}^{+}, S_{1}^{-}<S_{1}^{+}$, and $S_{0}^{ \pm} \leq$ $S_{1}^{ \pm}$, we have $0<U_{i}^{ \pm}(x)<1$ in $\Omega_{i}$ and $\pm\left(U_{1}^{ \pm}(x)-U_{0}^{ \pm}(x)\right) \leq 0$ in $\Omega_{0} \cap \Omega_{1}$, where $U_{i}^{ \pm}(x)=U^{ \pm}\left(S_{i}^{-}, S_{i}^{+} ; x\right)$ in $\Omega_{i}=\Omega\left(S_{i}^{-}, S_{i}^{+}\right)$.

Proof. This follows from the maximum and comparison principles for harmonic functions.

3.4. Lemma. Let $U(x)=U^{+}\left(S^{-}, S^{+} ; x\right)$ in $C l(\Omega)$ where $\Omega=\Omega\left(S^{-}, S^{+}\right), S^{ \pm} \in \mathbb{X}$, and $S^{-}<S^{+}$. Then:

(a) We have $U(x) \geq\left(x \cdot \nu_{0}-\alpha\right) /(\beta-\alpha)$ in $\Omega \cap\left\{\alpha<x \cdot \nu_{0}<\beta\right\}$ for any unit vector $\nu_{0}$ and values $\alpha<\beta$ such that $S^{-} \subset\left\{x \cdot \nu_{0} \leq \alpha\right\}$ and $S^{+} \subset\left\{x \cdot \nu_{0} \leq \beta\right\}$.

(b) We have that

$$
U(x) \geq\left(d\left(x, S^{-}\right) / \Delta\left(S^{-}, S^{+}\right)\right)
$$

and

$$
(1-U(x)) \leq\left(d\left(x, S^{+}\right) / d\left(S^{-}, S^{+}\right)\right)
$$

both in $\Omega$. 
(c) For any $\alpha \in(0,1)$, the real-analytic surface $S_{\alpha}:=\{U(x)=\alpha\}$ is convex. It $i s$ also strictly convex in the sense that any supporting plane intersects $S_{\alpha}$ at only one point.

(d) The function $Q(x):=|\nabla U(x)|$ is weakly decreasing (with increasing $U$ ) on all the curves of steepest ascent of $U$.

(e) The function $Q(x):=|\nabla U(x)|$ is subharmonic in $\Omega$, and the function $W(x)$ $:=g(Q(x))$ is superharmonic in $\Omega$ provided that $g(t): \mathbb{R}_{+} \rightarrow \mathbb{R}$ is a $C^{2}$-function such that $d\left(t g^{\prime}(t)\right) / d t \leq 0$, where $g^{\prime}(t)=d g / d t \geq 0$ (this includes the case where $g(t)=\ln (t))$.

(f) For $0 \leq \alpha<\beta \leq 1$, let $x_{\alpha} \in S_{\alpha}$ and $x_{\beta} \in S_{\beta}$ be the endpoints of an arc $\gamma$ of steepest ascent of $U$. Then $Q\left(x_{\beta}\right)|\gamma| \leq(\beta-\alpha) \leq Q\left(x_{\alpha}\right)|\gamma|$ if the derivatives exist, where $|\gamma|$ denotes the arc-length of $\gamma$.

(g) For any unit vector $\nu_{0}$, any values $0 \leq \alpha<\beta \leq 1$, and any points $x_{\alpha} \in S_{\alpha}$ and $x_{\beta} \in S_{\beta}$ such that $\left(x-x_{\alpha}\right) \cdot \nu_{0} \leq 0$ for all $x \in S_{\alpha}$ and $\left(x-x_{\beta}\right) \cdot \nu_{0} \leq 0$ for all $x \in S_{\beta}$, we have that $Q\left(x_{\beta}\right) \leq((\beta-\alpha) / d(\alpha, \beta)) \leq Q\left(x_{\alpha}\right)$ if the derivatives exist, where $d(\alpha, \beta)=\left(x_{\beta}-x_{\alpha}\right) \cdot \nu_{0}$.

Proof sketch. Part (a) follows from the comparison principle, and both inequalities in Part (b) follow from Part (a) (the first inequality in Part (b) also follows from the fact that $d\left(x, S^{-}\right)$is subharmonic in $\left.\Omega\right)$. Regarding Part (c), see [14], [15], §2, [17], [19], or [22]. Part (d) is a direct consequence of Part (c) and the Laplace equation. The assertions in Part (e) also follow from Part (c), as is seen by a direct computation of $\Delta Q$ and $\Delta W$ (observe that $Q>0$ in $\Omega$, essentially due to part (d)). Finally, Part (f) follows from Part (d), and Part (g) is obtained by applying Part (a) to the capacitary potential $(U(x)-\alpha) /(\beta-\alpha)$ in the domain $\Omega\left(S_{\alpha}, S_{\beta}\right)$.

3.5. Lemma. For any $S^{ \pm} \in \mathbb{X}$ such that $\partial B_{\delta}\left(x_{0}\right) \leq S^{-}<S^{+} \leq \partial B_{R}\left(x_{0}\right)$ (for some $0<\delta<R$ and $\left.x_{0} \in \mathbb{R}^{N}\right)$, we have $\Delta^{-}\left(S^{-}, S^{+}\right) \leq \Delta^{+}\left(S^{-}, S^{+}\right) \leq$ $(R / \delta) \Delta^{-}\left(S^{-}, S^{+}\right)$, where we define $\Delta^{ \pm}\left(S^{-}, S^{+}\right)=\max \left\{d\left(x, S^{\mp}\right): x \in S^{ \pm}\right\}$. Therefore, $\Delta\left(S^{-}, S^{+}\right)=\Delta^{+}\left(S^{-}, S^{+}\right)$and $\Delta\left(S^{-}, S^{+}\right) \leq(R / \delta) \Delta^{-}\left(S^{-}, S^{+}\right)$.

(We leave this proof to the reader.)

3.6. Lemma. Let $S_{0}^{ \pm}, S_{1}^{ \pm} \in \mathbb{X}$ denote convex surfaces such that $S_{0}^{-}<S_{0}^{+}, S_{1}^{-}<$ $S_{1}^{+}$, and $S_{0}^{ \pm} \leq S_{1}^{ \pm}$. Then for any $\varepsilon \in(0,1)$, we have that: (a) $\Phi_{\varepsilon}^{ \pm}\left(S_{i}^{-}, S_{i}^{+}\right) \in$ $\mathbb{X},(\mathrm{b}) \Phi_{\varepsilon}^{ \pm}\left(S_{0}^{-}, S_{0}^{+}\right) \leq \Phi_{\varepsilon}^{ \pm}\left(S_{1}^{-}, S_{1}^{+}\right)$, (c) $\Psi_{\varepsilon}\left(S_{i}^{-}, S_{i}^{+}\right) \in \mathbb{X}$, (d) $\Psi_{\varepsilon}\left(S_{0}^{-}, S_{0}^{+}\right) \leq$ $\Psi_{\varepsilon}\left(S_{1}^{-}, S_{1}^{+}\right)$, (e) if the surface $S_{0}^{-}$is strictly convex, then the function $\phi_{0}(x):=$ $f\left(x,\left(d\left(x, S_{0}^{-}\right) / \varepsilon\right),\left(d\left(x, S_{0}^{+}\right) / \varepsilon\right)\right)$ is strictly positive in the exterior complement of the surface $S_{0}:=\Psi_{\varepsilon}\left(S_{0}^{-}, S_{0}^{+}\right)$relative to the annular domain $\Omega_{0}:=\Omega\left(S_{0}^{-}, S_{0}^{+}\right)$. Therefore $S_{0}=\left\{x \in \Omega_{0}: \phi_{0}(x)=0\right\}$.

Proof. Parts (a) and (b) follow from Lemmas 3.4(c) and 3.3. Parts (c) and (d) are given in [11], Lemma 3.8 and Theorem 4.3(b), and Part (e) follows from a slight generalization of the proof of [11], Theorem 4.3(b).

3.7. Lemma. Let $S, S^{ \pm}, \widehat{S}, \widehat{S}^{ \pm} \in \mathbb{X}$ denote convex surfaces such that $S^{-}<S<$ $S^{+}, \widehat{S}^{-}<\widehat{S}<\widehat{S}^{+}, S \leq \hat{S}$, and $S^{ \pm} \leq \widehat{S}^{ \pm}$. Then for any $\varepsilon \in(0,1)$, we have that: (a) $T_{\varepsilon}\left(S^{-}, S, S^{+}\right) \in \mathbb{X}$, and (b) $T_{\varepsilon}\left(S^{-}, S, S^{+}\right) \leq T_{\varepsilon}\left(\widehat{S}^{-}, \widehat{S}, \widehat{S}^{+}\right)$.

Proof. This proof follows from Lemma 3.6 (see [11], Theorem 3.3 and Lemma 3.9).

3.8. Lemma. The surface $T_{\varepsilon}\left(S_{1}, S, S_{2}\right) \in \mathbb{X}$ depends continuously on $S_{1}, S_{2}, S \in \mathbb{X}$ (such that $S_{1}<S<S_{2}$ ) and on $\varepsilon \in(0,1)$. 
Proof sketch. Assume for $n \rightarrow \infty$ that $S_{1, n} \rightarrow S_{1}, S_{n} \rightarrow S$, and $S_{2, n} \rightarrow S_{2}$ in $\mathbb{X}$ (where $S_{1}<S<S_{2}$ ) and $\varepsilon_{n} \rightarrow \varepsilon \in(0,1)$. It follows from the continuity of $F(x, p, q)$ and well-known properties of harmonic functions that $U^{ \pm}\left(S_{1, n}, S_{2, n} ; x\right) \rightarrow$ $U^{ \pm}\left(S_{1}, S_{2} ; x\right)$ and

$$
\begin{aligned}
\phi_{n}(x) & :=F\left(x,\left(\varepsilon_{n} / d\left(x, S_{1, n}\right)\right),\left(\varepsilon_{n} / d\left(x, S_{2, n}\right)\right) \rightarrow \phi(x)\right. \\
& :=F\left(x,\left(\varepsilon / d\left(x, S_{1}\right)\right),\left(\varepsilon / d\left(x, S_{2}\right)\right),\right.
\end{aligned}
$$

both as $n \rightarrow \infty$, where the convergence is uniform in any particular compact subset of $\Omega:=\Omega\left(S_{1}, S_{2}\right)$. The first convergence result implies that for each point $x$ in the interior (exterior) complement of $\Phi^{ \pm}\left(S_{1}, S_{2} ; \varepsilon\right)$ relative to $\Omega$, the value $\pm\left(U^{ \pm}\left(S_{1, n}, S_{2, n} ; x\right)-\varepsilon_{n}\right)$ is negative (positive) if $n \in \mathbb{N}$ is sufficiently large. In view of Lemma 3.6(e), the second convergence result implies that if $S_{1}$ is strictly convex, then for each point $x$ in the interior (exterior) complement of $\Psi\left(S_{1}, S_{2} ; \varepsilon\right)$ relative to $\Omega$, the value $\phi_{n}(x)$ is negative (positive) if $n \in \mathbb{N}$ is sufficiently large. It follows that $\Phi^{ \pm}\left(S_{1, n}, S_{2, n} ; \varepsilon_{n}\right) \rightarrow \Phi^{ \pm}\left(S_{1}, S_{2} ; \varepsilon\right)$ as $n \rightarrow \infty$, and that $\Psi\left(S_{1, n}, S_{2, n} ; \varepsilon\right) \rightarrow$ $\Psi\left(S_{1}, S_{2} ; \varepsilon\right)$ as $n \rightarrow \infty$, provided that $S_{1}$ is strictly convex in the second case. Since the surfaces $\Phi^{ \pm}\left(S_{1}, S_{2} ; \varepsilon\right)$ are in fact strictly convex, it follows from (3.1) that $T\left(S_{1, n}, S_{n}, S_{2, n} ; \varepsilon_{n}\right) \rightarrow T\left(S_{1}, S, S_{2} ; \varepsilon\right)$ as $n \rightarrow \infty$.

3.9. Fixed-point problem. Given $\varepsilon \in(0,1)$ and surfaces $S^{ \pm} \in \mathbb{X}$ with $S^{-}<S^{+}$, we seek surfaces $S_{\varepsilon} \in \mathbb{X}$ such that $S^{-}<S_{\varepsilon}<S^{+}$and $T_{\varepsilon}\left(S^{-}, S_{\varepsilon}, S^{+}\right)=S_{\varepsilon}$.

3.10. Definitions. Given $\varepsilon \in(0,1)$ and the surfaces $S^{ \pm} \in \mathbb{X}$ with $S^{-}<S^{+}$, we use the notation $\mathfrak{S}_{\varepsilon}\left(S^{-}, S^{+}\right)=\mathfrak{S}\left(S^{-}, S^{+} ; \varepsilon\right)$ to denote the family of all solutions of Problem 3.9. Also, $\mathfrak{S}_{\varepsilon}^{-}\left(S^{-}, S^{+}\right)$(resp. $\mathfrak{S}_{\varepsilon}^{+}\left(S^{-}, S^{+}\right)$) denotes the family of all surfaces $S \in \mathbb{X}$ such that $S^{-}<S<S^{+}$and such that $T_{\varepsilon}\left(S^{-}, S, S^{+}\right) \geq S$ (resp. $\left.T_{\varepsilon}\left(S^{-}, S, S^{+}\right) \leq S\right)$. Thus, for fixed $\varepsilon \in(0,1)$ and $S^{ \pm} \in \mathbb{X}, \mathfrak{S}_{\varepsilon}^{-}\left(S^{-}, S^{+}\right)$(resp. $\mathfrak{S}_{\varepsilon}^{+}\left(S^{-}, S^{+}\right)$) is the family of all inner (outer) solutions of the fixed-point problem 3.9. Clearly $\mathfrak{S}_{\varepsilon}\left(S^{-}, S^{+}\right)=\mathfrak{S}_{\varepsilon}^{-}\left(S^{-}, S^{+}\right) \cap \mathfrak{S}_{\varepsilon}^{+}\left(S^{-}, S^{+}\right)$.

3.11. Lemma. Assume that $S_{0} \in \mathfrak{S}_{\varepsilon}^{+}\left(S_{0}^{-}, S_{0}^{+}\right)$for a given value $\varepsilon \in(0,1)$, and for given surfaces $S_{0}^{ \pm} \in \mathbb{X}$. Then $S_{0} \in \mathfrak{S}_{\varepsilon}^{+}\left(S^{-}, S^{+}\right)$for any surfaces $S^{ \pm} \in \mathbb{X}$ such that $S^{-}<S_{0}<S^{+}$and $S^{ \pm} \leq S_{0}^{ \pm}$. Analogously, if $S_{0} \in \mathfrak{S}_{\varepsilon}^{-}\left(S_{0}^{-}, S_{0}^{+}\right)$, then $S_{0} \in \mathfrak{S}_{\varepsilon}^{-}\left(S^{-}, S^{+}\right)$for any surfaces $S^{ \pm} \in \mathbb{X}$ such that $S^{-}<S_{0}<S^{+}$and $S_{0}^{ \pm} \leq S^{ \pm}$.

Proof. This follows from Lemma 3.7(b).

3.12. Definition. A surface $\widetilde{S} \in \mathbb{X}$ (such that $S_{*}^{-}<\widetilde{S}<S_{*}^{+}$) is called a weak solution of Problem 2.1 if there exist a positive null sequence $\left(\varepsilon_{n}\right)_{n=1}^{\infty}$ and a corresponding sequence of ordered triples $\left(S_{*, n}^{-}, S_{n}, S_{*, n}^{+}\right)_{n=1}^{\infty}\left(\right.$ with $S_{*, n}^{-}<S_{n}<S_{*, n}^{+}$ in $\mathbb{X})$ such that $\Delta\left(S_{n}, \widetilde{S}\right) \rightarrow 0$ and $\Delta\left(S_{*, n}^{ \pm}, S_{*}^{ \pm}\right) \rightarrow 0$, both as $n \rightarrow \infty$, and such that for each $n \in \mathbb{N}, S_{n}$ solves Problem 3.9 in the particular case where $\varepsilon$ and $S^{ \pm}$ are replaced respectively by $\varepsilon_{n}$ and $S_{*, n}^{ \pm}$(i.e., we have $S_{n} \in \mathfrak{S}\left(S_{*, n}^{-}, S_{*, n}^{+} ; \varepsilon_{n}\right)$ ). The sequence of ordered triples is called an approximating sequence corresponding to the weak solution $\widetilde{S}$.

3.13. Remarks. (a) Our definition of a weak solution is based essentially on the fact that if $\widetilde{S}$ is a sufficiently regular classical solution of Problem 2.1, then

$$
\Delta\left(T_{\varepsilon}\left(S_{*}^{-}, \widetilde{S}, S_{*}^{+}\right), \widetilde{S}\right)=\varepsilon z(\varepsilon) \quad \text { as } \varepsilon \downarrow 0,
$$

so that $\widetilde{S}$ nearly a solution of Problem 3.9 for small $\varepsilon \in(0,1)$ (see [11], Lemma 3.13). (b) The above definition of a weak solution is slightly more general than 
necessary (in the present context), in order to accommodate the requirements of the multilayer problem in $\S 5$.

3.14. Lemma. Given the surfaces $S^{ \pm} \in \mathbb{X}$ with $S^{-}<S^{+}$, let $S_{\alpha}=\left\{x \in D\left(S^{+}\right)\right.$: $\left.d\left(x, S^{+}\right)=\alpha\right\} \in \mathbb{X}$ for $0<\alpha<d\left(S^{-}, S^{+}\right)$. Then for fixed, sufficiently small $\alpha$, we have $S_{\alpha} \in \mathfrak{S}_{\varepsilon}^{+}\left(S^{-}, S^{+}\right)$for all $\varepsilon \in(0,1)$.

Proof. We have $S_{\alpha, \varepsilon}:=T_{\varepsilon}\left(S^{-}, S_{\alpha}, S^{+}\right)<S_{\alpha}$ provided that

$$
f\left(x,\left[d\left(x, S_{\alpha, \varepsilon}^{-}\right) / \varepsilon\right],\left[d\left(x, S_{\alpha, \varepsilon}^{+}\right) / \varepsilon\right]\right)>0
$$

for all $x \in S_{\alpha}$, where $S_{\alpha, \varepsilon}^{ \pm}=\Phi_{\varepsilon}^{ \pm}\left(S_{\alpha}, S^{ \pm}\right)$. However, it follows from Lemmas $3.4(\mathrm{~b})$ and 3.5 that $\left(d\left(x, S_{\alpha, \varepsilon}^{+}\right) / \varepsilon\right) \leq \Delta^{+}\left(S_{\alpha}, S^{+}\right) \leq(R / \delta) \alpha$ and $\left(d\left(x, S_{\alpha, \varepsilon}^{-}\right) / \varepsilon\right) \geq$ $d\left(S^{-}, S_{\alpha}\right) \geq\left(d\left(S^{-}, S^{+}\right) / 2\right)$, both whenever $0<\varepsilon<1$ and $0<\alpha<\left(d\left(S^{-}, S^{+}\right) / 2\right)$, where we choose $x_{0} \in D\left(S^{-}\right)$and $0<\delta<R$ such that $\partial B_{\delta}\left(x_{0}\right) \leq S^{-}<S^{+} \leq$ $\partial B_{R}\left(x_{0}\right)$. The assertion follows in view of Assumption 2.2(b).

3.15. Lemma. Let $S^{ \pm} \in \mathbb{X}$ denote fixed surfaces such that $S^{-}<S^{+}$. Let $S_{\alpha}=$ $\Phi_{\alpha}^{+}\left(S^{-}, S^{+}\right) \in \mathbb{X} \cap C^{\infty}$ for $\alpha \in(0,1)$. Then there exist a value $\alpha_{0}>0$ and a function $\varepsilon_{0}(\alpha):\left(0, \alpha_{0}\right] \rightarrow \mathbb{R}$ such that $S_{\alpha} \in \mathfrak{S}_{\varepsilon}^{-}\left(S^{-}, S^{+}\right)$for $\alpha \in\left(0, \alpha_{0}\right]$ and $\varepsilon \in\left(0, \varepsilon_{0}(\alpha)\right]$.

Proof. One can easily verify that $U_{\alpha}^{-}(x)=((\alpha-U(x)) / \alpha)$ and $\left|\nabla U_{\alpha}^{-}(x)\right|=(Q(x) / \alpha)$ both in $\Omega_{\alpha}^{-}$, and $U_{\alpha}^{+}(x)=((U(x)-\alpha) /(1-\alpha))$ and $\left|\nabla U_{\alpha}^{+}(x)\right|=(Q(x) /(1-\alpha))$ both in $\Omega_{\alpha}^{+}$, where $U(x)=U^{+}\left(S^{-}, S^{+} ; x\right), \Omega=\Omega\left(S^{-}, S^{+}\right), Q(x)=|\nabla U(x)|, U_{\alpha}^{ \pm}(x)=$ $U^{ \pm}\left(S^{ \pm}, S_{\alpha} ; x\right)$ and $\Omega_{\alpha}^{ \pm}=\Omega\left(S^{ \pm}, S_{\alpha}\right)$. Also, we have $S_{\alpha}<S_{\alpha, \varepsilon}:=T_{\varepsilon}\left(S^{-}, S_{\alpha}, S^{+}\right)$ provided that

$$
f\left(x,\left[d\left(x, S_{\alpha, \varepsilon}^{-}\right) / \varepsilon\right],\left[d\left(x, S_{\alpha, \varepsilon}^{+}\right) / \varepsilon\right]\right)<0
$$

for all $x \in S_{\alpha}$ where $S_{\alpha, \varepsilon}^{ \pm}=\Phi_{\varepsilon}^{ \pm}\left(S^{ \pm}, S_{\alpha}\right)$. Also $\left[d\left(x, S_{\alpha, \varepsilon}^{-}\right) / \varepsilon\right]=(\alpha / Q(x))+z_{\alpha}^{-}(x, \varepsilon)$ and $\left[d\left(x, S_{\alpha, \varepsilon}^{+}\right) / \varepsilon\right]=((1-\alpha) / Q(x))+z_{\alpha}^{+}(x, \varepsilon)$, both for all $x \in S_{\alpha}$ by the theorem of the mean, where $z_{\alpha}^{ \pm}(x, \varepsilon) \rightarrow 0$ as $\varepsilon \downarrow 0$, uniformly over all $x \in S_{\alpha}$ for each $\alpha \in(0,1)$. It follows that $S_{\alpha}<S_{\alpha, \varepsilon}$ provided that

$$
f\left(x,(\alpha / Q(x))+z_{\alpha}^{-}(x, \varepsilon),((1-\alpha) / Q(x))+z_{\alpha}^{+}(x, \varepsilon)\right)<0
$$

for all $x \in S_{\alpha}$. Now (3.2) holds for all sufficiently small $\varepsilon \in(0,1)$ (depending on $\alpha$ ) provided that

$$
f(x,(\alpha / Q(x)),((1-\alpha) / Q(x)))<0
$$

for all $x \in S_{\alpha}$. Since $\min \{Q(x): U(x) \leq 1 / 2\}>0$, the assertion now follows from Assumption 2.2(c).

3.16. Lemma. Assume that $S_{\varepsilon}^{-} \leq S_{\varepsilon}^{+}$, where $S_{\varepsilon}^{ \pm} \in \mathfrak{S}_{\varepsilon}^{ \pm}\left(S^{-}, S^{+}\right)$. Then there exists at least one solution $S_{\varepsilon} \in \mathfrak{S}_{\varepsilon}\left(S^{-}, S^{+}\right)$of Problem 3.9 such that $S_{\varepsilon}^{-} \leq S_{\varepsilon} \leq$ $S_{\varepsilon}^{+}$.

Proof. Define the sequence $\left(S_{\varepsilon, n}\right)_{n=1}^{\infty} \subset \mathbb{X}$ such that $S_{\varepsilon, 0}=S_{\varepsilon}^{+}$and $S_{\varepsilon, n+1}=$ $T_{\varepsilon}\left(S^{-}, S_{\varepsilon, n}, S^{+}\right) \in \mathbb{X}$ for $n=0,1,2, \ldots$ It follows inductively from Lemma $3.7(\mathrm{~b})$ that $S_{\varepsilon}^{+} \geq S_{\varepsilon, n} \geq S_{\varepsilon, n+1} \geq S_{\varepsilon}^{-}$for $n=0,1,2, \ldots$ Therefore, $S_{\varepsilon, n} \downarrow S_{\varepsilon} \in \mathbb{X}$ as $n \rightarrow \infty$, where (by Lemma 3.8):

$$
T_{\varepsilon}\left(S^{-}, S_{\varepsilon}, S^{+}\right)=T_{\varepsilon}\left(S^{-}, \lim _{n \rightarrow \infty} S_{\varepsilon, n}, S^{+}\right)=\lim _{n \rightarrow \infty} S_{\varepsilon, n+1}=S_{\varepsilon} .
$$


3.17. Proof of Theorem 2.3(a). According to Lemmas 3.14 and 3.15, there exist a value $\varepsilon_{0} \in(0,1)$ and fixed surfaces $\widehat{S}^{ \pm} \in \mathbb{X}$ such that $S_{*}^{-} \leq \widehat{S}^{-}<\widehat{S}^{+}<S_{*}^{+}$and such that $\widehat{S}^{ \pm} \in \mathfrak{S}_{\varepsilon}^{ \pm}\left(S_{*}^{-}, S_{*}^{+}\right)$for all $\varepsilon \in\left(0, \varepsilon_{0}\right]$. It follows by Lemma 3.16 that for any $\varepsilon \in\left(0, \varepsilon_{0}\right]$, there exists a solution $S_{\varepsilon}$ of Problem 3.9 at $\varepsilon$ such that $\widehat{S}^{-} \leq S_{\varepsilon} \leq \widehat{S}^{+}$. Given a null sequence $\left(\varepsilon_{n}\right)_{n=1}^{\infty}$ such that $\varepsilon_{n} \in\left(0, \varepsilon_{0}\right]$ for each $n$, let $\left(S_{n}\right)_{n=1}^{\infty}$ denote a sequence such that for each $n \in \mathbb{N}, S_{n} \in \mathfrak{S}\left(S_{*}^{-}, S_{*}^{+} ; \varepsilon_{n}\right)$ denotes a solution of Problem 3.9 in the case where $S^{ \pm}=S_{*}^{ \pm}$and $\varepsilon=\varepsilon_{n}$. The surfaces $S_{n}$ have equicontinuous, uniformly bounded polar-coordinate representations relative to a fixed origin located anywhere in $D\left(S_{*}^{-}\right)$. Therefore, by the theorem of Ascoli-Arzela, we can pass to an appropriate subsequence (still indexed by $n \in \mathbb{N}$ ) such that $S_{n} \rightarrow$ $\widetilde{S}$ (in the polar-coordinate maximum norm) as $n \rightarrow \infty$, where $\widetilde{S}$ denotes a particular limit surface (with a continuous polar-coordinate representation). Clearly, $\widetilde{S} \in$ $\mathbb{X}, \widehat{S}^{-} \leq \widetilde{S} \leq \widehat{S}^{+}$, and $\Delta\left(S_{n}, \widetilde{S}\right) \rightarrow 0$ as $n \rightarrow \infty$. Thus, $\widetilde{S}$ is a weak solution of Problem 2.1.

\section{UNIFORM RESULTS INVOLVING SOLUTIONS OF THE TWO-LAYER FIXED POINT PROBLEM}

The purpose of the present section is to develop some uniform estimates for solutions of the two-layer fixed-point problem (Problem 3.9) which will be essential in our study of the multilayer fixed-point problem and the multilayer free-boundary problem.

4.1. Lemma. For given constants $0<\delta_{0}<R_{0}$ and any given unit vector $\nu_{0}$, define $K_{r}=r K$ and $S_{r}^{ \pm}=r S^{ \pm}$, and $\Omega_{r}=r \Omega$ for any $r \in(0,1]$, where $K=$ $H\left(\{0\} \cup C l\left(B_{\lambda}\left(\delta_{0} \nu_{0}\right)\right)\right), \lambda=\left(\delta_{0}^{2} / R_{0}\right), S^{-}=\partial K, S^{+}=\{x \notin K: d(x, K)=1\}$, and $\Omega=\Omega\left(S^{-}, S^{+}\right)$. Also define $U_{r}(x)=U^{+}\left(S_{r}^{-}, S_{r}^{+} ; x\right)$ in $\Omega_{r}, S_{r, \alpha}=\Phi_{\alpha}^{+}\left(S_{r}^{-}, S_{r}^{+}\right)=$ $\left\{U_{r}(x)=\alpha\right\}$, and $S_{r, \alpha, \varepsilon}=T_{\varepsilon}\left(S_{r}^{-}, S_{r, \alpha}, S_{r}^{+}\right)$for $r \in(0,1]$ and $\alpha, \varepsilon \in(0,1)$. Then there exist a value $\alpha_{0} \in(0,1)$ and a positive, continuous function $\varepsilon_{0}(\alpha):\left(0, \alpha_{0}\right] \rightarrow$ $\mathbb{R}$ (neither of which depends on the unit vector $\left.\nu_{0}\right)$ such that if $\alpha \in\left(0, \alpha_{0}\right]$ and $\varepsilon \in\left(0, \varepsilon_{0}(\alpha)\right]$, then $S_{r, \alpha}<S_{r, \alpha, \varepsilon}$ for all $r \in(0,1]$.

Proof. For any $r \in(0,1]$ and $\alpha, \varepsilon \in(0,1)$, we have that $S_{r, \alpha}=r S_{\alpha}$, where $S_{\alpha}=\Phi_{\alpha}^{+}\left(S^{-}, S^{+}\right)$. By a change of scale, we also have that $S_{r, \alpha, \varepsilon}=r \hat{S}_{r, \alpha, \varepsilon}=$ $r T_{r, \varepsilon}\left(S^{-}, S_{\alpha}, S^{+}\right)$, where we define

$$
\begin{aligned}
\widehat{S}_{r, \alpha, \varepsilon} & =T_{r, \varepsilon}\left(S^{-}, S_{\alpha}, S^{+}\right) \\
& :=\left\{x \in \Omega\left(S_{\alpha, \varepsilon}^{-}, S_{\alpha, \varepsilon}^{+}\right): f_{r}\left(x,\left[d\left(x, S_{\alpha, \varepsilon}^{-}\right) / \varepsilon\right],\left[d\left(x, S_{\alpha, \varepsilon}^{+}\right) / \varepsilon\right]\right)=0\right\},
\end{aligned}
$$

for all $r \in(0,1]$, with $S_{\alpha, \varepsilon}^{ \pm}=\Phi_{\varepsilon}^{ \pm}\left(S^{ \pm}, S_{\alpha}\right)$ and $f_{r}(x, p, q)=f(r x, r p, r q)$. Clearly $S_{r, \alpha, \varepsilon}>S_{r, \alpha}$ for $r \in(0,1]$ if and only if $\widehat{S}_{r, \alpha, \varepsilon}>S_{\alpha}$ for $r \in(0,1]$. A sufficient condition for the latter is that

$$
f\left(r x, r\left[d\left(x, S_{\alpha, \varepsilon}^{-}\right) / \varepsilon\right], r\left[d\left(x, S_{\alpha, \varepsilon}^{+}\right) / \varepsilon\right]\right)<0
$$

for all $r \in(0,1]$ and $x \in S_{\alpha}$. However, by the argument given in the proof of Lemma 3.15, (4.1) holds if we have

$$
\sup \left\{f(y,(\alpha r / Q(x)),((1-\alpha) r / Q(x))): x \in S_{\alpha}, r \in(0,1], y \in \mathbb{R}^{N}\right\}<0
$$

for sufficiently small $\alpha>0$ (i.e., for $\alpha \in\left(0, \alpha_{0}\right]$, where $\alpha_{0}>0$ is sufficiently small), where $Q(x)=|\nabla U|$ and $U(x)=U^{+}\left(S^{-}, S^{+} ; x\right)$. However, this follows from Assumption 2.2(c). 
4.2. Corollary. Let the constants $0<\delta_{0}<R_{0}$ be fixed. For all values $\alpha \in(0,1)$ and $r \in(0,1]$, and for all points $x_{0} \neq 0$, define $S_{r}^{-}\left(x_{0}\right)=\partial K_{r}\left(x_{0}\right)$ and $S_{r}^{+}\left(x_{0}\right)=$ $\left\{x \notin K_{r}\left(x_{0}\right): d\left(x, K_{r}\left(x_{0}\right)\right)=r\right\}$, where $K_{r}\left(x_{0}\right)=H\left(\left\{x_{0}\right\} \cup C l\left[B_{\lambda r}\left(p_{0}\left(x_{0}, r\right)\right)\right]\right), \lambda=$ $\left(\delta_{0}^{2} / R_{0}\right)$, and $p_{0}\left(x_{0}, r\right)=\left(\left(\left|x_{0}\right|-\delta_{0} r\right) /\left|x_{0}\right|\right) x_{0}$. Then there exist a value $\alpha_{0} \in(0,1)$ and a positive, continuous function $\varepsilon_{0}(\alpha):\left(0, \alpha_{0}\right] \rightarrow \mathbb{R}$ (neither of which depends on the unit vector $\left.\nu_{0}\right)$ such that if $\alpha \in\left(0, \alpha_{0}\right]$ and $\varepsilon \in\left(0, \varepsilon_{0}(\alpha)\right]$, then

$$
S_{r, \alpha, \varepsilon}\left(x_{0}\right):=T_{\varepsilon}\left(S^{-}\left(x_{0}\right), S_{r, \alpha}\left(x_{0}\right), S_{r}^{+}\left(x_{0}\right)\right)>S_{r, \alpha}\left(x_{0}\right):=\Phi_{\alpha}^{+}\left(S_{r}^{-}\left(x_{0}\right), S_{r}^{+}\left(x_{0}\right)\right)
$$

uniformly for all $x_{0} \neq 0$ and $r \in(0,1]$.

Proof. For each $x_{0} \neq 0$, the surfaces $S^{ \pm}\left(x_{0}\right)$ and $S_{r, \alpha}\left(x_{0}\right)$ are obtained from the corresponding surfaces $S_{r}^{ \pm}$and $S_{r, \alpha}$ defined in Lemma 4.1 (in the specific case where $\left.\nu_{0}=-\left(x_{0} /\left|x_{0}\right|\right)\right)$ by applying the transformation $y=y(x)=x-x_{0}$. Therefore, the assertion follows from Lemma 4.1 by replacing $f(x, p, q)$ by the function $\hat{f}(x, p, q):=$ $f\left(x-x_{0}, p, q\right)$.

4.3. Remark. It is obvious in the context of Lemma 4.1 that there exists a null function $z_{0}(\cdot)$ such that $U(x):=U^{+}\left(S^{-}, S^{+} ; x\right) \leq z_{0}(|x|)$ in $\Omega$, and that there exists a positive function $p(t):(0,1) \rightarrow \mathbb{R}$ such that $d\left(S^{-}, S_{\alpha, \varepsilon}^{-}\right) \geq p(\alpha(1-\varepsilon))$ for each $\alpha, \varepsilon \in(0,1)$, where $S_{\alpha, \varepsilon}^{-}:=\Phi_{\varepsilon}^{-}\left(S^{-}, S_{\alpha}\right)=\{U(x)=\alpha(1-\varepsilon)\}$. By rescaling, we have $U_{r}\left(x_{0} ; x\right) \leq z_{0}\left(\left|x-x_{0}\right| / r\right)$ in $\Omega_{r}\left(x_{0}\right)$ and $d\left(S_{r}^{-}\left(x_{0}\right), S_{r, \alpha, \varepsilon}^{-}\left(x_{0}\right)\right) \geq p(\alpha(1-\varepsilon)) r$, both uniformly for all $x_{0} \neq 0$ and all $r \in(0,1]$, where $S_{r, \alpha, \varepsilon}^{-}\left(x_{0}\right):=\Phi_{\varepsilon}^{-}\left(S_{r}^{-}\left(x_{0}\right), S_{r, \alpha}\left(x_{0}\right)\right)$, and $U_{r}\left(x_{0} ; x\right)=U^{+}\left(S_{r}^{-}\left(x_{0}\right), S_{r}^{+}\left(x_{0}\right) ; x\right)$ in $\Omega_{r}\left(x_{0}\right):=\Omega\left(S_{r}^{-}\left(x_{0}\right), S_{r}^{+}\left(x_{0}\right)\right)$.

4.4. Lemma. Let $\mathbb{X}_{0}=\left\{S \in \mathbb{X}: \partial B\left(0 ; \delta_{0}\right) \leq S \leq \partial B\left(0 ; R_{0}\right)\right\}$, where the constants $0<\delta_{0}<R_{0}$ are given. Then there exist constants $\eta_{0}>0$ and $\varepsilon_{0} \in(0,1)$ such that $d\left(S^{-}, S_{\varepsilon}^{-}\right) \geq \eta_{0} d\left(S^{-}, S^{+}\right)$for any $\varepsilon \in\left(0, \varepsilon_{0}\right]$, any surfaces $S^{ \pm} \in \mathbb{X}_{0}$ satisfying $S^{-}<S^{+}$, and any surface $S_{\varepsilon} \in \mathfrak{S}_{\varepsilon}^{+}\left(S^{-}, S^{+}\right)$, where we define $S_{\varepsilon}^{-}=\Phi_{\varepsilon}^{-}\left(S^{-}, S_{\varepsilon}\right)$.

Proof. In the context of Lemma 4.1 and Corollary 4.2 , choose $\varepsilon_{0}=\varepsilon_{0}(\alpha)$, where $\alpha \in\left(0, \alpha_{0}\right]$ is fixed. Let $S_{\varepsilon}$ and $S^{ \pm}$be any fixed surfaces in $\mathbb{X}_{0}$ such that $S^{-}<$ $S_{\varepsilon}<S^{+}$and $\widehat{S}_{\varepsilon}:=T_{\varepsilon}\left(S^{-}, S_{\varepsilon}, S^{+}\right) \leq S_{\varepsilon}$. In the notation of Corollary 4.2, we have that $S_{r}^{-}\left(x_{0}\right) \leq S^{-}$for any $r \in(0,1]$ and $x_{0} \in S^{-}$, as follows from properties of $\mathbb{X}_{0}$. Choose the value $r>0$ to be maximum subject to the requirements that (a) $r \leq 1$, (b) $S_{r}^{+}\left(x_{0}\right) \leq S^{+}$for all $x_{0} \in S^{-}$, and (c) $S_{r, \alpha}\left(x_{0}\right) \leq S_{\varepsilon}$ for all $x_{0} \in S^{-}$. Then, by continuity, one of the following alternatives must hold: either (a) $r=1$, or (b) $S^{+} \cap S_{r}^{+}\left(x_{0}\right) \neq \varnothing$ for at least one point $x_{0} \in S^{-}$, or else (c) $S_{\varepsilon} \cap S_{r, \alpha}\left(x_{0}\right) \neq \varnothing$ for at least one point $x_{0} \in S^{-}$. However, Corollary 4.2 implies that

$$
S_{r, \alpha}\left(x_{0}\right)<S_{r, \alpha, \varepsilon}\left(x_{0}\right) \leq \widehat{S}_{\varepsilon}:=T_{\varepsilon}\left(S^{-}, S_{\varepsilon}, S^{+}\right) \leq S_{\varepsilon}
$$

for all $x_{0} \in S^{-}$, so that the alternative (c) is impossible. Therefore, one of the alternatives (a) and (b) holds. In other words, we must have that $S_{r, \alpha}\left(x_{0}\right) \leq S_{\varepsilon}$ for all $x_{0} \in S^{-}$, where $r=\min \left\{1, d\left(S^{-}, S^{+}\right)\right\}$. Due to Lemma 3.6(b), it follows that $S_{r, \alpha, \varepsilon}^{-}\left(x_{0}\right):=\Phi_{\varepsilon}^{-}\left(S^{-}\left(x_{0}\right), S_{r, \alpha}\left(x_{0}\right)\right) \leq S_{\varepsilon}^{-}:=\Phi_{\varepsilon}^{-}\left(S^{-}, S_{\varepsilon}\right)$. Therefore, we have

$$
d\left(x_{0}, S_{\varepsilon}^{-}\right) \geq d\left(x_{0}, S_{r, \alpha, \varepsilon}^{-}\left(x_{0}\right)\right) \geq p(\alpha(1-\varepsilon)) \min \left\{1, d\left(S^{-}, S^{+}\right)\right\}
$$

for all $x_{0} \in S^{-}$, where we have applied an estimate in Remark 4.3. This completes the proof, in view of the fact that there exists a uniform upper bound for the values $\left\{d\left(S^{-}, S^{+}\right): S^{ \pm} \in \mathbb{X}_{0}, S^{-}<S^{+}\right\}$. 
4.5. Corollary. In the context of Lemma 4.4, for any $\varepsilon \in\left(0, \varepsilon_{0}\right]$ and any surfaces $S^{ \pm} \in \mathbb{X}_{0}$ such that $S^{-}<S^{+}$, there exists a surface $\widehat{S}_{\varepsilon} \in \mathfrak{S}_{\varepsilon}\left(S^{-}, S^{+}\right)$such that $d\left(S^{-}, \widehat{S}_{\varepsilon}\right) \geq \eta_{0} d\left(S^{-}, S^{+}\right)$and such that $S \geq \widehat{S}_{\varepsilon}$ for all surfaces $S \in \mathfrak{S}_{\varepsilon}^{+}\left(S^{-}, S^{+}\right)$. We call $\widehat{S}_{\varepsilon}$ the minimal solution of Problem 3.9 at $\varepsilon$.

Proof. For fixed $\varepsilon \in\left(0, \varepsilon_{0}\right]$, let $\widehat{S}_{\varepsilon}=\partial \widehat{D}_{\varepsilon} \in \mathbb{X}$, where $\widehat{D}_{\varepsilon}$ denotes the intersect of the interior complements of all surfaces $S \in \mathfrak{S}_{\varepsilon}^{+}\left(S^{-}, S^{+}\right)$. Clearly, we have $d\left(\hat{S}_{\varepsilon}, S^{-}\right) \geq \eta_{0} d\left(S^{-}, S^{+}\right)$, due to Lemma 4.4. For $x_{0} \in \widehat{S}_{\varepsilon}$, and for any $n \in \mathbb{N}$, there exist an outer solution $S_{n} \in \mathfrak{S}_{\varepsilon}^{+}\left(S^{-}, S^{+}\right)$and a point $x_{n} \in S_{n}$ such that $\left|x_{n}-x_{0}\right|<1 / n$. Since $S_{n} \geq \widehat{S}_{\varepsilon}$, it follows (by Lemma 3.6(b)) that $S_{n, \varepsilon}^{ \pm} \geq \widehat{S}_{\varepsilon}^{ \pm}$for all $n \in \mathbb{N}$, where $S_{n, \varepsilon}^{ \pm}:=\Phi_{\varepsilon}^{ \pm}\left(S_{n}, S^{ \pm}\right)$and $\widehat{S}_{\varepsilon}^{ \pm}:=\Phi_{\varepsilon}^{ \pm}\left(\widehat{S}_{\varepsilon}, S^{ \pm}\right)$. Therefore, we have $\pm\left[d\left(x_{n}, S_{n, \varepsilon}^{ \pm}\right)-d\left(x_{n}, \widehat{S}_{\varepsilon}^{ \pm}\right)\right] \geq 0$ for sufficiently large $n \in \mathbb{N}$ (so that $x_{n} \in \Omega\left(\widehat{S}_{\varepsilon}^{-}, \widehat{S}_{\varepsilon}^{+}\right)$). Therefore,

$$
\begin{gathered}
f\left(x_{0},\left[d\left(x_{0}, \widehat{S}_{\varepsilon}^{-}\right) / \varepsilon\right],\left[d\left(x_{0}, \widehat{S}_{\varepsilon}^{+}\right) / \varepsilon\right]\right)=\lim _{n \rightarrow \infty} f\left(x_{n},\left[d\left(x_{n}, \widehat{S}_{\varepsilon}^{-}\right) / \varepsilon\right],\left[d\left(x_{n}, \widehat{S}_{\varepsilon}^{+}\right) / \varepsilon\right]\right) \\
\geq \liminf _{n \rightarrow \infty} f\left(x_{n},\left[d\left(x_{n}, S_{n, \varepsilon}^{-}\right) / \varepsilon\right],\left[d\left(x_{n}, S_{n, \varepsilon}^{+}\right) / \varepsilon\right]\right) \geq 0 .
\end{gathered}
$$

Since $x_{0}$ is arbitrary in $\widehat{S}_{\varepsilon}$, it follows that $T_{\varepsilon}\left(S^{-}, \widehat{S}_{\varepsilon}, S^{+}\right) \leq \widehat{S}_{\varepsilon}$, i.e. $\widehat{S}_{\varepsilon} \in \mathfrak{S}_{\varepsilon}^{+}\left(S^{-}, S^{+}\right)$. Therefore $T_{\varepsilon}\left(S^{-}, \widehat{S}_{\varepsilon}, S^{+}\right) \in \mathfrak{S}_{\varepsilon}^{+}\left(S^{-}, S^{+}\right)$, by Lemma 3.7. Therefore $T_{\varepsilon}\left(S^{-}, \widehat{S}_{\varepsilon}, S^{+}\right)$ $\geq \widehat{S}_{\varepsilon}$, due to the minimality of $\widehat{S}_{\varepsilon}$ in $\mathfrak{S}_{\varepsilon}^{+}\left(S^{-}, S^{+}\right)$. Therefore $T_{\varepsilon}\left(S^{-}, \widehat{S}_{\varepsilon}, S^{+}\right)=\widehat{S}_{\varepsilon}$, whence $\widehat{S}_{\varepsilon} \in \mathfrak{S}_{\varepsilon}\left(S^{-}, S^{+}\right)$.

4.6. Lemma. Let $\mathbb{X}_{1}=\left\{S \in \mathbb{X}: S \leq \partial B\left(0 ; R_{0}\right)\right\}$. Then there exists a positive, continuous, increasing function $h(r): \mathbb{R}_{+} \rightarrow \mathbb{R}_{+}$with the following property: For any values $\varepsilon \in(0,1), \delta>0, \alpha \in(0, h(\delta)]$ and any surfaces $S^{ \pm}, S_{\varepsilon} \in \mathbb{X}_{1}$ such that $S^{-}<S^{+}, d\left(S^{-}, S^{+}\right) \geq \delta, S_{\varepsilon} \in \mathfrak{S}_{\varepsilon}^{-}\left(S^{-}, S^{+}\right)$, and $d\left(S_{\varepsilon}, S^{+}\right) \geq \alpha \delta$, we have $d\left(S_{\varepsilon}, S^{+}\right) \geq \alpha d\left(S^{-}, S^{+}\right)$. Under the same assumptions, we also have $d\left(S_{\varepsilon}^{+}, S^{+}\right) \geq$ $(\alpha / 2) d\left(S^{-}, S^{+}\right)$for $\varepsilon \in(0,1 / 2]$, where $S_{\varepsilon}^{+}:=\Phi_{\varepsilon}^{+}\left(S_{\varepsilon}, S^{+}\right)$.

Proof. This proof is based on a comparison to the operator method in the limiting case in which the convex surfaces become parallel planes. Let $x_{0}, \nu_{0} \in \mathbb{R}^{N}$ (such that $\left.\left|\nu_{0}\right|=1\right)$ be fixed. For any $r>0$, define $S_{r}^{+}=\{\phi(x)=0\}, S_{r}^{-}=\{\phi(x)=$ $r\}, S_{r, \alpha}=\{\phi(x)=\alpha r\}, U_{r, \alpha}^{-}(x)=((\phi(x)-\alpha r) /(1-\alpha) r)$ in $\Omega_{r, \alpha}^{-}:=\{\alpha r<$ $\phi(x)<r\}, U_{r, \alpha}^{+}(x)=((\alpha r-\phi(x)) / \alpha r)$ in $\Omega_{r, \alpha}^{+}:=\{0<\phi(x)<\alpha r\}$, where $\phi(x)=$ $\left(x_{0}-x\right) \cdot \nu_{0}$. Then $T_{\varepsilon}\left(S_{r}^{-}, S_{r, \alpha}, S_{r}^{+}\right)<S_{r, \alpha}$ (where the ordering " $<$ " is defined relative to the direction $\left.\nu_{0}\right)$ for all $\varepsilon \in(0,1)$ provided that $f(x,(1-\alpha) r, \alpha r)>0$ for all $x \in S_{r, \alpha}$. However, for any given value $\delta>0$, there exists a value $h(\delta)>0$ (which does not depend on $x_{0}$ or $\left.\nu_{0}\right)$ so small that $f(x,(1-\alpha) r, \alpha r)>0$ for all $x \in \mathbb{R}^{N}, r \in\left[\delta, 2 R_{0}\right]$, and $\alpha \in(0, h(\delta)]$. Thus, for any fixed $\alpha \in(0, h(\delta)]$, we have $\widehat{S}_{r, \varepsilon}:=T_{\varepsilon}\left(S_{r}^{-}, \widehat{S}_{r}, S_{r}^{+}\right)<\widehat{S}_{r}$ for all $\varepsilon \in(0,1)$ and $r \in\left[\delta, 2 R_{0}\right]$, where $\widehat{S}_{r}:=S_{r, \alpha}$. For fixed $\alpha \in(0, h(\delta)]$ and $\varepsilon \in(0,1)$, let $S^{ \pm}$and $S_{\varepsilon}$ be any fixed surfaces in $\mathbb{X}_{1}$ such that $S^{-}<S_{\varepsilon}<S^{+}, \widehat{S}_{\varepsilon}:=T_{\varepsilon}\left(S^{-}, S_{\varepsilon}, S^{+}\right) \geq S_{\varepsilon}, d\left(S^{-}, S^{+}\right) \geq \delta$, and $d\left(S_{\varepsilon}, S^{+}\right) \geq$ $\alpha \delta$. Let $M$ denote the set of all pairs $\left(x_{0}, \nu_{0}\right)$ such that $x_{0} \in S^{+},\left|\nu_{0}\right|=1$, and $\left(x_{0}-z\right) \cdot \nu_{0} \geq 0$ for all $z \in D\left(S^{+}\right)$. For any $r>0$, let $S_{r}^{ \pm}\left(x_{0}, \nu_{0}\right), \widehat{S}_{r}\left(x_{0}, \nu_{0}\right)$, and $\widehat{S}_{r, \varepsilon}\left(x_{0}, \nu_{0}\right)$ denote the surfaces $S_{r}^{ \pm}, \widehat{S}_{r}$, and $\widehat{S}_{r, \varepsilon}$ corresponding to each particular choice of $\left(x_{0}, \nu_{0}\right) \in M$. For any $\left(x_{0}, \nu_{0}\right) \in M$, it is easily verified that $S^{+} \leq$ $S_{r}^{+}\left(x_{0}, \nu_{0}\right)$ for all $r>0$, and that $S^{-} \leq S_{r}^{-}\left(x_{0}, \nu_{0}\right)$ and $S_{\varepsilon} \leq \widehat{S}_{r}\left(x_{0}, \nu_{0}\right)$, both for all $r \in(0, \delta]$. Now choose the value $r>0$ to be maximum subject to the requirement 
that $S^{-} \leq S_{r}^{-}\left(x_{0}, \nu_{0}\right)$ and $S_{\varepsilon} \leq \widehat{S}_{r}\left(x_{0}, \nu_{0}\right)$. Then $r \in\left[\delta, 2 R_{0}\right]$, and by continuity, one of the following alternatives must hold: either (a) $S^{-} \cap S_{r}^{-}\left(x_{0}, \nu_{0}\right) \neq \varnothing$ for at least one pair $\left(x_{0}, \nu_{0}\right) \in M$, or else (b) $S_{\varepsilon} \cap \widehat{S}_{r}\left(x_{0}, \nu_{0}\right) \neq \varnothing$ for at least one pair $\left(x_{0}, \nu_{0}\right) \in M$. However, since $r \in\left[\delta, 2 R_{0}\right]$, it follows by (a slight generalization of) Lemma 3.7(b) that

$$
S_{\varepsilon} \leq \widehat{S}_{\varepsilon} \leq \widehat{S}_{r, \varepsilon}\left(x_{0}, \nu_{0}\right) \leq \widehat{S}_{r}\left(x_{0}, \nu_{0}\right)
$$

for all $\left(x_{0}, \nu_{0}\right) \in M$, so that the alternative (b) is impossible. Therefore (a) holds, implying that $r \geq d\left(S^{-}, S^{+}\right)$. Therefore, $d\left(S_{\varepsilon}, S^{+}\right) \geq \alpha d\left(S^{-}, S^{+}\right)$, as was asserted.

4.7. Corollary. In the context of Lemma 4.6, for any $\delta>0$, there exists a value $\varepsilon_{0}=\varepsilon_{0}(\delta) \in(0,1)$ such that for any $\varepsilon \in\left(0, \varepsilon_{0}\right]$ and any surfaces $S^{ \pm}, S_{\varepsilon} \in \mathbb{X}_{1}$ such that $S^{-}<S^{+}, d\left(S^{-}, S^{+}\right) \geq \delta, S_{\varepsilon} \in \mathfrak{S}_{\varepsilon}^{-}\left(S^{-}, S^{+}\right)$, and $d\left(S_{\varepsilon}, S^{+}\right) \geq \delta h(\delta / 2)$, there exists a surface $\widehat{S}_{\varepsilon} \in \mathfrak{S}_{\varepsilon}\left(S^{-}, S^{+}\right)$such that $\widehat{S}_{\varepsilon} \geq S_{\varepsilon}$ and $d\left(\widehat{S}_{\varepsilon}, S^{+}\right) \geq \delta h(\delta / 2)$.

Proof. Define the sequence $\left(S_{\varepsilon, n}\right)_{n=1}^{\infty}$ inductively such that $S_{\varepsilon, 1}=S_{\varepsilon}$ and $S_{\varepsilon, n+1}=$ $T_{\varepsilon}\left(S^{-}, S_{\varepsilon, n}, S^{+}\right)$for all $n \in \mathbb{N}$. By Lemma 3.7, we have $S_{\varepsilon, n} \in \mathfrak{S}_{\varepsilon}^{-}\left(S^{-}, S^{+}\right)$and $S_{\varepsilon, n} \leq S_{\varepsilon, n+1}<S^{+}$for all $n \in \mathbb{N}$. Assuming that $d\left(S_{\varepsilon, n}, S^{+}\right) \geq \delta h(\delta / 2)$ for some $n \in \mathbb{N}$ (as is certainly true in the case $n=1$ ), we have $d\left(S_{\varepsilon, n+1}, S^{+}\right) \geq(\delta / 2) h(\delta / 2)$ for sufficiently small $\varepsilon>0$. Since $S_{\varepsilon, n+1} \in \mathfrak{S}_{\varepsilon}^{-}\left(S^{-}, S^{+}\right)$, it follows from Lemma 4.6 that actually $d\left(S_{\varepsilon, n+1}, S^{+}\right) \geq \delta h(\delta / 2)$. Therefore, if $\varepsilon>0$ is sufficiently small, then $d\left(S_{\varepsilon, n}, S^{+}\right) \geq \delta h(\delta / 2)$ for all $n \in \mathbb{N}$. Therefore, $S_{\varepsilon, n} \uparrow \widehat{S}_{\varepsilon} \in \mathfrak{S}_{\varepsilon}\left(S^{-}, S^{+}\right)$, where $d\left(\widehat{S}_{\varepsilon}, S^{+}\right) \geq \delta h(\delta / 2)$.

4.8. Lemma. Let Assumptions 2.2(a)-(c) hold. Given constants $0<\delta_{0}<R_{0}$, let $\mathbb{X}_{0}=\left\{S \in \mathbb{X}: \partial B\left(0 ; \delta_{0}\right) \leq S \leq \partial B\left(0 ; R_{0}\right)\right\}$. Then for any given value $d_{0}>0$, there exist constants $0<\mathfrak{C}_{0} \leq 1 \leq \mathfrak{C}_{1}<\infty$ such that for any $\varepsilon \in(0,1)$, any surfaces $S^{ \pm} \in \mathbb{X}_{0}$ such that $S^{-}<S^{+}$, and any surface $S_{\varepsilon} \in \mathfrak{S}_{\varepsilon}\left(S^{-}, S^{+}\right)$such that $d\left(S^{ \pm}, S_{\varepsilon}^{ \pm}\right) \geq 2 d_{0}$ (where $\left.S_{\varepsilon}^{ \pm}:=\Phi_{\varepsilon}^{ \pm}\left(S^{ \pm}, S_{\varepsilon}\right)\right)$, we have $\mathfrak{C}_{0} \leq\left(\varepsilon / d\left(x_{0}, S_{\varepsilon}^{ \pm}\right)\right) \leq$ $\mathfrak{C}_{1}$ for all $x_{0} \in S_{\varepsilon}$. Moreover, we have $Q_{\varepsilon}^{-}(x) \leq \mathfrak{C}_{1}$ in $\Omega\left(S_{0}^{-}, S_{\varepsilon}\right), Q_{\varepsilon}^{+}(x) \leq$ $\mathfrak{C}_{1}$ in $\Omega\left(S_{\varepsilon}^{+}, S^{+}\right), Q_{\varepsilon}^{+}(x) \geq \mathfrak{C}_{0}$ in $\Omega\left(S_{\varepsilon}, S_{0}^{+}\right)$, and $Q_{\varepsilon}^{-}(x) \geq \mathfrak{C}_{0}$ in $\Omega\left(S^{-}, S_{\varepsilon}^{-}\right)$, where $U_{\varepsilon}^{ \pm}(x)=U^{ \pm}\left(S^{ \pm}, S_{\varepsilon} ; x\right), Q_{\varepsilon}^{ \pm}(x)=\left|\nabla U_{\varepsilon}^{ \pm}(x)\right|$, and $S_{0}^{ \pm}=\left\{x \in \Omega\left(S^{-}, S^{+}\right)\right.$: $\left.d\left(x, S^{ \pm}\right)=d_{0}\right\}$.

Proof. Let an admissible pair $S^{ \pm} \in \mathbb{X}_{0}$ be fixed. We first obtain the uniform upper bounds for $Q_{\varepsilon}^{ \pm}(x)$ and $\left(\varepsilon / d\left(x_{0}, S_{\varepsilon}^{ \pm}\right)\right.$) (with $x_{0} \in S_{\varepsilon}$ ). For any $\varepsilon \in(0,1)$ and solution $S_{\varepsilon} \in \mathfrak{S}_{\varepsilon}\left(S^{-}, S^{+}\right)$such that $d\left(S^{-}, S_{\varepsilon}^{-}\right) \geq 2 d_{0}$, it follows from Lemma 3.4 (f) that $Q_{\varepsilon}^{-}(x) \leq\left(1 / d\left(x, S^{-}\right)\right) \leq B_{1}:=\left(1 / d_{0}\right)$ for all $x \in \Omega\left(S^{-}, S_{\varepsilon}\right)$ such that $d\left(x_{0}, S^{-}\right) \geq d_{0}$. Therefore $\left(\varepsilon / d\left(x_{0}, S_{\varepsilon}^{-}\right)\right) \leq\left(\varepsilon / d\left(S_{\varepsilon}^{-}, S_{\varepsilon}\right)\right) \leq Q_{\varepsilon}^{-}\left(x_{1}\right) \leq B_{1}$, by Lemma $3.4(\mathrm{~g})$, where $x_{0} \in S_{\varepsilon}$ and $x_{1} \in S_{\varepsilon}^{-}$is chosen such that $d\left(x_{1}, S_{\varepsilon}\right)=$ $d\left(S_{\varepsilon}^{-}, S_{\varepsilon}\right)$. Since $F\left(x_{0},\left[\varepsilon / d\left(x_{0}, S_{\varepsilon}^{-}\right)\right],\left[\varepsilon / d\left(x_{0}, S_{\varepsilon}^{+}\right)\right]\right)=0$, it follows by Assumption 2.2 (a) that $F\left(x_{0}, B_{1},\left[\varepsilon / d\left(x_{0}, S_{\varepsilon}^{+}\right)\right]\right) \leq 0$ for all $x_{0} \in S_{\varepsilon}$. Therefore, $\left(\varepsilon / d\left(x_{0}, S_{\varepsilon}^{+}\right)\right) \leq$ $\mathfrak{C}_{1}$ for $x_{0} \in S_{\varepsilon}$ by Assumption 2.2(b), where we define $\mathfrak{C}_{1}=\max \left\{1, B_{1}, q_{0}\left(B_{1}\right)\right\} \geq$ $B_{1}$. Therefore $d\left(x_{1}, S_{\varepsilon}\right) \geq\left(\varepsilon / \mathfrak{C}_{1}\right)$ for $x_{1} \in S_{\varepsilon}^{+}$, from which it follows by Lemma $3.4(\mathrm{f})$ that $Q_{\varepsilon}^{+}(x) \leq \mathfrak{C}_{1}$ on $S_{\varepsilon}^{+}$. Thus $Q_{\varepsilon}^{+}(x) \leq \mathfrak{C}_{1}$ throughout $\Omega\left(S_{\varepsilon}^{+}, S^{+}\right)$, by Lemma 3.4(d).

We now turn to the proof of the uniform, positive lower bounds for $Q_{\varepsilon}^{ \pm}(x)$ and $\left(\varepsilon / d\left(x_{0}, S_{\varepsilon}^{ \pm}\right)\right)$(with $\left.x_{0} \in S_{\varepsilon}\right)$. Given $\varepsilon \in(0,1)$ and a solution $S_{\varepsilon} \in \mathfrak{S}_{\varepsilon}\left(S^{-}, S^{+}\right)$ such that $d\left(S^{+}, S_{\varepsilon}^{+}\right) \geq 2 d_{0}$, we have $\left(x_{1}-x_{0}\right) \cdot \nu_{0} \leq R_{0}$ for any $x_{0} \in \Omega\left(S_{\varepsilon}, S^{+}\right)$ and $x_{1} \in S^{+}$, where $\nu_{0}=\nu\left(x_{0}\right)=\nabla U_{\varepsilon}^{+}\left(x_{0}\right) / Q_{\varepsilon}^{+}\left(x_{0}\right)$. Therefore, $Q_{\varepsilon}^{+}\left(x_{0}\right) \geq$ 
$\left(1-U_{\varepsilon}^{+}\left(x_{0}\right)\right) / R_{0}$ for $x_{0} \in \Omega\left(S_{\varepsilon}, S^{+}\right)$, due to Lemma 3.4(g). On the other hand, for any $x_{0} \in \Omega\left(S_{\varepsilon}, S_{0}^{+}\right)$, we have that $S^{-}\left(x_{0}\right) \leq S_{\varepsilon}, S^{+}\left(x_{0}\right) \leq S^{+}$, and $(1-$ $\left.U_{\varepsilon}^{+}(x)\right) \geq\left(1-U^{+}\left(x_{0} ; x\right)\right)$ for all $x \in \Omega\left(S_{\varepsilon}, S^{+}\right) \cap \Omega\left(S^{-}\left(x_{0}\right), S^{+}\left(x_{0}\right)\right)$ (by Lemma $3.3)$, where $S^{-}\left(x_{0}\right)=\partial B\left(0 ; \delta_{0} / 2\right), S^{+}\left(x_{0}\right)=\partial H\left(\bar{B}\left(0 ; \delta_{0}\right) \cup\left\{\left(1+\left(d_{0} /\left|x_{0}\right|\right) x_{0}\right)\right\}\right)$, and $U^{+}\left(x_{0} ; x\right)=U^{+}\left(S^{-}\left(x_{0}\right), S^{+}\left(x_{0}\right) ; x\right)$. However, the continuous function $\phi\left(x_{0}\right):=$ $\left(1-U^{+}\left(x_{0} ; x_{0}\right)\right):\left(\bar{B}\left(0 ; R_{0}\right) \backslash B\left(0 ; \delta_{0}\right)\right) \rightarrow \mathbb{R}$ is strictly positive (by the strict maximum principle for harmonic functions), and therefore uniformly bounded from below by a positive constant $C>0$. Therefore, $Q_{\varepsilon}^{+}\left(x_{0}\right) \geq B_{0}:=C / R_{0}$, uniformly for $\varepsilon \in(0,1), S_{\varepsilon} \in \mathfrak{S}_{\varepsilon}\left(S^{-}, S^{+}\right)$such that $d\left(S^{+}, S_{\varepsilon}^{+}\right) \geq 2 d_{0}$, and $x_{0} \in \Omega\left(S_{\varepsilon}, S_{0}^{+}\right)$ by Lemma $3.4(\mathrm{~g})$. Therefore, $\left(\varepsilon / d\left(x_{0}, S_{\varepsilon}^{+}\right)\right) \geq B_{0}$ for $x_{0} \in S_{\varepsilon}$, due to Lemma $3.4(\mathrm{f})$. Since $F\left(x_{0},\left[\varepsilon / d\left(x_{0}, S_{\varepsilon}^{-}\right)\right],\left[\varepsilon / d\left(x_{0}, S_{\varepsilon}^{+}\right)\right]\right)=0$ for $x_{0} \in S_{\varepsilon}$, it follows by Assumption 2.2(a) that $F\left(x_{0},\left[\varepsilon / d\left(x_{0}, S_{\varepsilon}^{-}\right)\right], B_{0}\right) \leq 0$ for $x_{0} \in S_{\varepsilon}$. Therefore, $\left(\varepsilon / d\left(x_{0}, S_{\varepsilon}^{-}\right)\right) \geq \mathfrak{C}_{0}:=\min \left\{1, B_{0}, p_{0}\left(B_{0}\right)\right\}>0$ for $x_{0} \in S_{\varepsilon}$, by Assumption 2.2(b). Thus, $Q_{\varepsilon}^{-}\left(x_{1}\right) \geq \mathfrak{C}_{0}$ for all $x_{1} \in S_{\varepsilon}^{-}$, due to Lemma 3.4(g). Finally, we have $Q_{\varepsilon}^{-}(x) \geq \mathfrak{C}_{0}$ throughout $\Omega\left(S^{-}, S_{\varepsilon}^{-}\right)$, by Lemma $3.4(\mathrm{~d})$.

4.9. Corollary. Let $\widetilde{S} \in \mathbb{X}$ denote a (fixed) weak solution of Problem 2.1, where we assume $\S 2.2(\mathrm{a})-(\mathrm{c})$. Then there exist positive constants $0<\mathfrak{C}_{0} \leq \mathfrak{C}_{1}<\infty$ (depending on $\left.d_{0}:=(1 / 3) \min \left\{d\left(S_{*}^{-}, \widetilde{S}\right), d\left(\widetilde{S}, S_{*}^{+}\right)\right\}\right)$such that $\mathfrak{C}_{0} \leq\left|\nabla \widetilde{U}^{ \pm}(x)\right| \leq \mathfrak{C}_{1}$ uniformly over all $x \in \widetilde{\Omega}^{ \pm}$such that $d\left(x, S_{*}^{ \pm}\right) \geq d_{0}$, where we define $\widetilde{U}^{ \pm}(x)=$ $U^{ \pm}\left(S_{*}^{ \pm}, \widetilde{S} ; x\right)$ in $\widetilde{\Omega}^{ \pm}:=\Omega\left(S_{*}^{ \pm}, \widetilde{S}\right)$.

Proof. Let $\left(S_{*, n}^{-}, S_{n}, S_{*, n}^{+}\right)_{n=1}^{\infty}$ be an approximating sequence corresponding to a positive null sequence $\left(\varepsilon_{n}\right)_{n=1}^{\infty}$. Since $d\left(\widetilde{S}, S_{*}^{ \pm}\right) \geq 3 d_{0}$, we have $d\left(S_{n}, S_{*, n}^{ \pm}\right) \geq 2 d_{0}$ for all sufficiently large $n \in \mathbb{N}$. Since $S_{n} \in \mathfrak{S}\left(S_{*, n}^{-}, S_{*, n}^{+} ; \varepsilon_{n}\right)$ for each $n \in \mathbb{N}$, the assertions of Lemma 4.8 apply to $\left(S_{*, n}^{-}, S_{n}, S_{*, n}^{+}\right)$for all sufficiently large $n \in \mathbb{N}$. The present assertions follow from this, in view of the fact that $U_{n}^{ \pm} \rightarrow \widetilde{U}^{ \pm}$and $\nabla U_{n}^{ \pm} \rightarrow \nabla \widetilde{U}^{ \pm}$, both uniformly relative to all compact subsets of $\widetilde{\Omega}^{ \pm}$, where we define $U_{n}^{ \pm}(x)=U^{ \pm}\left(S_{n}^{ \pm}, S_{n} ; x\right)$ in $\Omega_{n}^{ \pm}:=\Omega\left(S_{n}^{ \pm}, S_{n}\right)$.

4.10. Lemma. In the context of Lemma 4.8, there exist a value $\alpha_{0} \in(0,1)$ and a positive, continuous, monotone increasing function $z_{1}(t):[0, \infty) \rightarrow \mathbb{R}$ (with $\left.z_{1}(0)=0\right)$, such that for any $\varepsilon \in(0,1 / 2]$ and $\alpha \in\left(0, \alpha_{0}\right]$, any surfaces $S^{ \pm} \in \mathbb{X}_{0}$ such that $S^{-}<S^{+}$, and any $S_{\varepsilon} \in \mathfrak{S}_{\varepsilon}\left(S^{-}, S^{+}\right)$such that $d\left(S^{ \pm}, S_{\varepsilon}^{ \pm}\right) \geq 2 d_{0}$, we have:

$$
\begin{gathered}
\left(\mathfrak{C}_{0} \delta_{0} / R_{0}\right) d\left(x, S_{\varepsilon}\right) \leq U_{\varepsilon}^{+}(x) \leq \varepsilon z_{1}\left(d\left(x, S_{\varepsilon}\right) / \varepsilon\right) \text { in } \Omega\left(S_{\varepsilon}, S_{\varepsilon}^{+}\right) . \\
Q_{\varepsilon}^{+}(x) \leq \mathfrak{C}^{+}(\alpha):=\left(2 N / z_{1}^{-1}(\alpha)\right) \text { in } C l\left(\Omega\left(S_{\varepsilon, \alpha}^{+}, S_{\varepsilon}^{+}\right)\right),
\end{gathered}
$$

where $U_{\varepsilon}^{+}(x):=U^{+}\left(S_{\varepsilon}, S^{+} ; x\right)$ and $S_{\varepsilon, \alpha}^{+}=\left\{U_{\varepsilon}^{+}(x)=\alpha \varepsilon\right\}$.

Proof. Since $\mathfrak{C}_{0} d\left(x, S_{\varepsilon}^{+}\right) \leq \varepsilon$ for $x \in S_{\varepsilon}$, by Lemma 4.8, it follows by Lemma 3.5 that $\mathfrak{C}_{0} d\left(x, S_{\varepsilon}\right) \leq\left(R_{0} / \delta_{0}\right) \varepsilon$ for all $x \in S_{\varepsilon}^{+}$. Thus, the first inequality in (4.2) holds for all $x \in S_{\varepsilon} \cup S_{\varepsilon}^{+}$. Therefore, it holds throughout $\Omega\left(S_{\varepsilon}, S_{\varepsilon}^{+}\right)$, since $d\left(x, S_{\varepsilon}\right)$ is subharmonic there. Turning to the second inequality in (4.2), we have $U_{\varepsilon}^{+}(x) \leq \varepsilon$ in $\Omega\left(S_{\varepsilon}, S_{\varepsilon}^{+}\right)$. Thus (in terms of notation from Corollary 4.2 and Remark 4.3), for any $x_{0} \in S_{\varepsilon}$, we have $U_{\varepsilon}^{+}(x) \leq \varepsilon U_{r}\left(x_{0} ; x\right)$ in $\omega\left(x_{0}\right):=\Omega\left(S_{\varepsilon}, S^{+}\right) \cap \Omega_{r}\left(x_{0}\right)$ for $r=r\left(x_{0}\right)=\min \left\{1, d\left(x_{0}, S_{\varepsilon}^{+}\right)\right\}$, since the inequality holds on $\partial \omega\left(x_{0}\right)$. Now $U_{r}\left(x_{0} ; x\right) \leq z_{0}\left(\left|x-x_{0}\right| / r\right)$ in $\Omega_{r}\left(x_{0}\right)$ for each $x_{0} \in S_{\varepsilon}$, due to Remark 4.3. Moreover, we have $r=r\left(x_{0}\right) \geq\left(\varepsilon / \mathfrak{C}_{1}\right)$ for $x_{0} \in S_{\varepsilon}$, due to Lemma 4.8. Thus, $U_{r}\left(x_{0} ; x\right) \leq$ $z_{0}\left(\mathfrak{C}_{1}\left|x-x_{0}\right| / \varepsilon\right)$ in $\Omega_{r}\left(x_{0}\right)$ for each $x_{0} \in S_{\varepsilon}$, from which the assertion follows. 
Turning to the proof of (4.3), it follows from Lemma 4.8 and Lemma 3.4(g) that $d\left(x, \widehat{S}_{\varepsilon}^{+}\right) \geq\left(\varepsilon / \mathfrak{C}_{1}\right)$ for $x \in \Omega\left(S_{\varepsilon}, S_{\varepsilon}^{+}\right)$and $\varepsilon \in(0,1 / 2]$, where $\widehat{S}_{\varepsilon}^{+}=\left\{U_{\varepsilon}^{+}(y)=\right.$ $2 \varepsilon\}$. Let $\alpha_{0}=\min \left\{1, z_{1}\left(1 / \mathfrak{C}_{1}\right)\right\}>0$. For any $\alpha \in\left(0, \alpha_{0}\right]$, and for any point $x \in \Omega\left(S_{\varepsilon}, S_{\varepsilon}^{+}\right)$such that $U_{\varepsilon}^{+}(x) \geq \alpha \varepsilon$, we have $\alpha \varepsilon \leq z_{1}\left(d\left(x, S_{\varepsilon}\right) / \varepsilon\right)$ by $(4.2)$, from which it follows that

$$
d\left(x, S_{\varepsilon} \cup \widehat{S}_{\varepsilon}^{+}\right) \geq \min \left\{d\left(x, S_{\varepsilon}\right), d\left(x, \widehat{S}_{\varepsilon}^{+}\right)\right\} \geq \varepsilon \min \left\{z_{1}^{-1}(\alpha),\left(1 / \mathfrak{C}_{1}\right)\right\}=\varepsilon z_{1}^{-1}(\alpha) .
$$

Therefore if $x \in \Omega\left(S_{\varepsilon, \alpha}^{+}, S_{\varepsilon}^{+}\right)$, then

$$
Q_{\varepsilon}^{+}(x) \leq N\left(\sup \left\{U_{\varepsilon}^{+}(y): y \in \Omega\left(S_{\varepsilon}, \widehat{S}_{\varepsilon}^{+}\right)\right\} / d\left(x, S_{\varepsilon} \cup \widehat{S}_{\varepsilon}^{+}\right)\right) \leq \mathfrak{C}^{+}(\alpha) .
$$

4.11. Lemma. In the context of Lemma 4.8, there exists a positive, continuous, monotone increasing function $z_{2}(t):[0, \infty) \rightarrow \mathbb{R}\left(\right.$ with $\left.z_{2}(0)=0\right)$, such that for any $\varepsilon \in\left(0, \varepsilon_{0}\right]$, any surfaces $S^{ \pm} \in \mathbb{X}_{0}$ such that $S^{-}<S^{+}$, and any $S_{\varepsilon} \in \mathfrak{S}_{\varepsilon}\left(S^{-}, S^{+}\right)$ such that $d\left(S^{ \pm}, S_{\varepsilon}^{ \pm}\right) \geq 2 d_{0}$, we have:

$$
\varepsilon z_{2}\left(d\left(x, S_{\varepsilon}\right) / \varepsilon\right) \leq U_{\varepsilon}^{-}(x) \leq \varepsilon \text { in } \Omega\left(S_{\varepsilon}^{-}, S_{\varepsilon}\right) .
$$

It follows from $(4.4 \mathrm{a})$ that

$$
d\left(x, S_{\varepsilon}\right) \leq \varepsilon z_{2}^{-1}(\alpha) \text { if } U_{\varepsilon}^{-}(x)=\alpha \varepsilon \text { for } \alpha \in(0,1] .
$$

Proof. For fixed constants $1<\mu<\lambda$, and for a variable unit vector $\nu_{0}$, let $\widehat{U}^{-}\left(\nu_{0} ; x\right)=U^{-}\left(\widehat{S}^{-}\left(\nu_{0}\right), \widehat{S}^{+}\left(\nu_{0}\right) ; x\right)$ in $\widehat{\Omega}\left(\nu_{0}\right):=\Omega\left(\widehat{S}^{-}\left(\nu_{0}\right), \widehat{S}^{+}\left(\nu_{0}\right)\right)$, where $\widehat{S}^{ \pm}\left(\nu_{0}\right)$ $=\partial K^{ \pm}\left(\nu_{0}\right), K^{+}\left(\nu_{0}\right)=H\left(\{0\} \cup \bar{B}_{\mu}\left(\lambda \nu_{0}\right)\right)$, and

$$
K^{-}\left(\nu_{0}\right)=H\left(\left\{\nu_{0}\right\} \cup \bar{B}_{((\lambda-1) / \lambda) \mu}\left(\lambda \nu_{0}\right)\right)=\left\{x \in K^{+}\left(\nu_{0}\right): d\left(x, \partial K^{+}\left(\nu_{0}\right)\right) \geq(\mu / \lambda)\right\} \text {. }
$$

Then $\hat{z}_{2}(t):=\widehat{U}^{-}\left(\nu_{0} ; t \nu_{0}\right)>0$ for $0<t \leq 1$, where this expression is independent of $\nu_{0}$, due to the congruence of the figures. For any $x_{0} \neq 0$ and small $r>0$, define $U_{r}^{-}\left(x_{0} ; x\right)=\widehat{U}^{-}\left(\nu_{0} ;\left(x-x_{0}\right) / r\right)$ for $x \in \Omega_{r}\left(x_{0}\right):=x_{0}+r \widehat{\Omega}\left(\nu_{0}\right)$, where $\nu_{0}=-\left(x_{0} /\left|x_{0}\right|\right)$. Observe that $U_{r}^{-}\left(x_{0} ; x_{0}+\nu_{0} t\right)=\hat{z}_{2}(t / r)$ for $0<t \leq r$, and that $\partial \Omega_{r}\left(x_{0}\right)=S_{r}^{-}\left(x_{0}\right) \cup S_{r}^{+}\left(x_{0}\right)$, where $S_{r}^{ \pm}\left(x_{0}\right):=x_{0}+r \widehat{S}^{ \pm}\left(\nu_{0}\right)\left(\right.$ and $\left.\nu_{0}=-\left(x_{0} /\left|x_{0}\right|\right)\right)$. For any $\varepsilon \in(0,1)$, surfaces $S_{\varepsilon}, S^{ \pm} \in \mathbb{X}_{0}$ such that $S_{\varepsilon} \in \mathfrak{S}_{\varepsilon}\left(S^{-}, S^{+}\right)$, and $x_{0} \in S_{\varepsilon}$, we conclude from Lemma 4.8 that $\left(\delta_{0} / R_{0}\right)\left|x_{0}^{-}-x_{0}\right| \leq d\left(x_{0}^{-}, S_{\varepsilon}\right) \leq\left(\varepsilon / \mathfrak{C}_{0}\right)$, where $x_{0}^{-}$denotes the point of intersection of $S_{\varepsilon}^{-}$with the line-segment joining $x_{0}$ to the origin. Using this, it is easily checked that if we choose $\lambda=\left(2 R_{0} / \delta_{0}\right)$ and $\mu=2$, then $S_{r}^{+}\left(x_{0}\right) \leq S_{\varepsilon}$ and $S_{r}^{-}\left(x_{0}\right) \leq S_{\varepsilon}^{-}$, both for all $x_{0} \in S_{\varepsilon}$ and $\varepsilon \in\left(0, \varepsilon_{1}\right]$, where $r=\left(R_{0} \varepsilon / \mathfrak{C}_{0} \delta_{0}\right)$ and $\varepsilon_{1}=\min \left\{\varepsilon_{0},\left(\mathfrak{C}_{0} \delta_{0}^{2} d_{0} / R_{0}^{2}\right)\right\}$. Thus, we have

(4.6) $U_{\varepsilon}^{-}\left(x_{0}+\nu_{0} t\right) \geq \varepsilon U_{r}^{-}\left(x_{0} ; x_{0}+\nu_{0} t\right)=\varepsilon \hat{z}_{2}(t / r) \geq \varepsilon \hat{z}_{2}\left(\mathfrak{C}_{0} \delta_{0} t / R_{0} \varepsilon\right)=: \varepsilon z_{2}(t / \varepsilon)$

for $x_{0} \in S_{\varepsilon}$ and $t \in(0, r]$ (where $\nu_{0}=-\left(x_{0} /\left|x_{0}\right|\right)$ ). Inequality (4.4a) follows from (4.6) and the fact that $d\left(x_{0}+t \nu_{0}, S_{\varepsilon}\right) \leq t$ for $t \in(0, r]$.

\section{The CONVEX MULTILAYER PROBLEM: FIXED POINTS AND WEAK SOLUTIONS}

5.1. Definitions, notation, assumptions. In the context of Problem 1.1 , let $\mathbb{Y}=$ $\mathbb{Y}\left(S_{*}^{-}, S_{*}^{+}\right)$denote the family of all $k$-tuples $S=\left(S_{1}, \ldots, S_{k}\right)$ such that $S_{i} \in \mathbb{X}$ for $i=1, \ldots, k$ and such that $S_{*}^{-}<S_{1}<\cdots<S_{k}<S_{*}^{+}$. For $S_{i}=\left(S_{i, 1}, \ldots, S_{i, k}\right) \in \mathbb{Y}$, $i=1,2$, we say $S_{1} \leq S_{2}$ if $S_{1, j} \leq S_{2, j}$ for $j=1, \ldots, k$. Also, we define $\Delta\left(S_{1}, S_{2}\right)=$ $\max \left\{\Delta\left(S_{1, j}, S_{2, j}\right): j=1, \ldots, k\right\}$, and we say that $S_{n} \rightarrow S$ as $n \rightarrow \infty$ (for $S, S_{n} \in$ $\mathbb{Y})$ if $\Delta\left(S_{n}, S\right) \rightarrow 0$ as $n \rightarrow \infty$. We assume throughout this section that the given functions $F_{i}(x, p, q): \mathbb{R}^{N} \times \mathbb{R}_{+} \times \mathbb{R}_{+} \rightarrow \mathbb{R}, i=1, \ldots, k$, satisfy Assumptions 2.2(a)(d). For each $i=1, \ldots, k$, the notations $T_{\varepsilon, i}\left(S_{i-1}, S_{i}, S_{i+1}\right), \mathfrak{S}_{\varepsilon, i}\left(S_{i-1}, S_{i+1}\right)$, and 
$\mathfrak{S}_{\varepsilon, i}^{ \pm}\left(S_{i-1}, S_{i+1}\right)$ coincide with $T_{\varepsilon}\left(S^{-}, S, S^{+}\right), \mathfrak{S}_{\varepsilon}\left(S^{-}, S^{+}\right)$, and $\mathfrak{S}_{\varepsilon}^{ \pm}\left(S^{-}, S^{+}\right)$in the case where $S=S_{i} \in \mathbb{X}, S^{ \pm}=S_{i \pm 1} \in \mathbb{X}$, and $F=F_{i}$ (here we define $S_{0}=$ $\left.S_{*}^{-}, S_{k+1}=S_{*}^{+}\right)$. Finally, we define the mappings $T_{\varepsilon}(S): \mathbb{Y} \rightarrow \mathbb{Y}, 0<\varepsilon<1$, such that $\left(T_{\varepsilon}(S)\right)_{i}=T_{\varepsilon, i}\left(S_{i-1}, S_{i}, S_{i+1}\right)$ for $i=1, \ldots, k$.

5.2. Problem. For any $\varepsilon \in(0,1)$, we seek a multisurface $S_{\varepsilon}:=\left(S_{\varepsilon, 1}, \ldots, S_{\varepsilon, k}\right) \in \mathbb{Y}$ such that $T_{\varepsilon}\left(S_{\varepsilon}\right)=S_{\varepsilon}$ (i.e., such that $S_{\varepsilon, i} \in \mathfrak{S}_{\varepsilon, i}\left(S_{\varepsilon, i-1}, S_{\varepsilon, i+1}\right)$ for $i=1,2, \ldots, k$, where $S_{\varepsilon, 0}=S_{*}^{-}$and $S_{\varepsilon, k+1}=S_{*}^{+}$).

5.3. Definitions. We define $\mathfrak{S}_{\varepsilon}^{ \pm}=\mathfrak{S}_{\varepsilon}^{ \pm}\left(S_{*}^{-}, S_{*}^{+}\right)=\left\{S=\left(S_{1}, \ldots, S_{k}\right) \in \mathbb{Y}: S_{i} \in\right.$ $\mathfrak{S}_{\varepsilon, i}^{ \pm}\left(S_{i-1}, S_{i+1}\right)$ for $\left.i=1, \ldots, k\right\}$ and $\mathfrak{S}_{\varepsilon}=\mathfrak{S}_{\varepsilon}\left(S_{*}^{-}, S_{*}^{+}\right)=\mathfrak{S}_{\varepsilon}^{-} \cap \mathfrak{S}_{\varepsilon}^{+}$for each $\varepsilon \in(0,1)$. We call $\mathfrak{S}_{\varepsilon}^{-}$(resp. $\mathfrak{S}_{\varepsilon}^{+}$) the family of inner (resp. outer) solutions of Problem 5.2 at $\varepsilon$, whereas $\mathfrak{S}_{\varepsilon}$ is the family of all solutions of Problem 5.2 at $\varepsilon$.

5.4. Definition. A multisurface $\widetilde{S}=\left(\widetilde{S}_{1}, \ldots, \widetilde{S}_{k}\right) \in \mathbb{Y}\left(S_{*}^{-}, S_{*}^{+}\right)$is a weak solution of Problem 1.1 if there exist a sequence of ordered $(k+2)$-tuples $\left(S_{*, n}^{-}, S_{n}, S_{*, n}^{+}\right)_{n=1}^{\infty}$ and a corresponding positive null sequence $\left(\varepsilon_{n}\right)_{n=1}^{\infty}$ such that for each $n \in \mathbb{N}$, we have $S_{n} \in \mathfrak{S}\left(S_{*, n}^{-}, S_{*, n}^{+} ; \varepsilon_{n}\right)$ (i.e. $S_{*, n}^{-}<S_{*, n}^{+}$in $\mathbb{X}, S_{n} \in \mathbb{Y}\left(S_{*, n}^{-}, S_{*, n}^{+}\right)$, and $S_{n}$ solves Problem 5.2 with $\varepsilon$ and $S_{*}^{ \pm}$replaced by $\varepsilon_{n}$ and $S_{*, n}^{ \pm}$, respectively), and such that $S_{*, n}^{ \pm} \rightarrow S_{*}^{ \pm}$and $S_{n} \rightarrow \widetilde{S}$ both as $n \rightarrow \infty$. The sequence $\left(S_{*, n}^{-}, S_{n}, S_{*, n}^{+}\right)_{n=1}^{\infty}$ is called an approximating sequence corresponding to the weak solution $\widetilde{S}$.

5.5. Theorem. There exist constants $\varepsilon_{0} \in(0,1)$ and $C>0$ such that for every $\varepsilon \in\left(0, \varepsilon_{0}\right]$, there exists at least one solution $S_{\varepsilon}:=\left(S_{\varepsilon, 1}, \ldots, S_{\varepsilon, k}\right) \in \mathfrak{S}_{\varepsilon}$ of Problem 5.2 at $\varepsilon$ such that $d\left(S_{\varepsilon, i}, S_{\varepsilon, i+1}\right) \geq C$ for $i=0, \ldots, k$.

5.6. Remarks. (a) As in the two-layer case, the intuitive justification for our definition of a weak solution of Problem 1.1 is based on the fact that if $\widetilde{S}$ is a sufficiently regular classical solution of Problem 1.1, then $\Delta\left(\widetilde{S}, T_{\varepsilon}(\tilde{S})\right)=\varepsilon z(\varepsilon)$ as $\varepsilon \downarrow 0$, so that $\widetilde{S}$ is nearly a solution of Problem 5.2 for small $\varepsilon>0$. (b) The remainder of this section is devoted mainly to the proofs of Theorems 5.5 and 2.11(a). For the proof of Theorem 5.5, we actually show (for aesthetic reasons) that the "fixed point" can be obtained as the limit of either a decreasing sequence of outer solutions (of Problem 5.2) or an increasing sequence of inner solutions, although obviously the study of either one of these two cases would suffice for establishing existence. The proof involving decreasing sequences of outer solutions is more straightforward.

\subsection{Lemma. There exist two fixed multisurfaces}

$$
S_{1}^{ \pm}:=\left(S_{1,1}^{ \pm}, \ldots, S_{1, n}^{ \pm}\right) \in \mathbb{Y}\left(S_{*}^{-}, S_{*}^{+}\right)
$$

such that $S_{1}^{ \pm} \in \mathfrak{S}_{\varepsilon}^{ \pm}\left(S_{*}^{-}, S_{*}^{+}\right)$for all $\varepsilon \in\left(0, \hat{\varepsilon}_{0}\right]$, where $\hat{\varepsilon}_{0} \in(0,1)$ is sufficiently small.

Proof. We will prove the existence of $S_{1}^{-}$. As an application of Lemma 3.15, we define the convex $C^{\infty}$-surfaces $S_{1, i}^{-}, i=1, \ldots, k$ inductively (as $i$ decreases, beginning with $i=k)$ such that $S_{1, i}^{-} \in \mathfrak{S}_{\varepsilon, i}^{-}\left(S_{*}^{-}, S_{1, i+1}^{-}\right)$for $\varepsilon \in\left(0, \varepsilon_{i}\right]$ and for each $i=1, \ldots, k$, where $S_{1, k+1}^{-}=S_{*}^{+}$. Since $S_{*}^{-}<S_{1,1}^{-}<\cdots<S_{1, k}^{-}<S_{*}^{-}$, it follows from Lemma 3.11 that $S_{1, i}^{-} \in \mathfrak{S}_{\varepsilon, i}^{-}\left(S_{1, i-1}^{-}, S_{1, i+1}^{-}\right)$for $i=1, \ldots, k$, provided that $0<\varepsilon \leq \hat{\varepsilon}_{0}:=\min \left\{\varepsilon_{1}, \ldots, \varepsilon_{k}\right\}$. The analogous construction of $S_{1}^{+}$is based on Lemma 3.14. 
5.8. Definitions. For $i=1, \ldots, k$, let $\eta_{i}^{+}>0$ and $\varepsilon_{0, i}^{+} \in(0,1)$ denote the constants $\eta_{0}$ and $\varepsilon_{0}$ of Lemma 4.4 in the case where $f=f_{i}$, and let $h_{i}(t)$ and $\varepsilon_{0, i}^{-}(t)$ denote the functions $h(t)$ and $\varepsilon_{0}(t)$ of Lemma 4.6 and Corollary 4.7 in the case where $f=f_{i}$. For any given, sufficiently small $\delta>0$, we define the values $\delta_{0}^{+}, \ldots, \delta_{k}^{+}, \delta_{1}^{-}, \ldots, \delta_{k+1}^{-}$, and $\eta_{1}^{ \pm}, \ldots, \eta_{k}^{ \pm}$recursively such that $\delta_{0}^{-}=\delta_{k+1}^{+}=\delta$ and $\delta_{i \mp 1}^{ \pm}=\eta_{i}^{ \pm} \delta_{i}^{ \pm}$for $i=1, \ldots, k$, where $\eta_{i}^{-}=h_{i}\left(\delta_{i}^{-} / 2\right)$ and $\eta_{i}^{+}$was defined previously. In terms of these values, we define $\tilde{\varepsilon}_{0}(\delta)=\min \left\{\varepsilon_{0,1}^{-}, \ldots, \varepsilon_{0, k}^{-}, \varepsilon_{0,1}^{+}, \ldots, \varepsilon_{0, k}^{+}, \hat{\varepsilon}_{0}\right\} \in$ $(0,1)$, where $\varepsilon_{0, i}^{-}:=\varepsilon_{0, i}^{-}\left(\delta_{i}^{-}\right) \in(0,1)$ for $i=1, \ldots, k$, the values $\varepsilon_{0,1}^{+}, \ldots, \varepsilon_{0, k}^{+}$were defined previously, and $\hat{\varepsilon}_{0}$ occurs in Lemma 5.7. We also define

$$
\begin{aligned}
& \mathbb{Y}_{\delta}^{+}=\left\{S=\left(S_{1}, \ldots, S_{k}\right) \in \mathbb{Y}: \begin{array}{l}
d\left(S_{k}, S_{*}^{+}\right) \geq \delta \text { and } \\
\left.d\left(S_{i-1}, S_{i}\right) \geq \eta_{i}^{+} d\left(S_{i-1}, S_{i+1}\right) \text { for } i=1, \ldots, k\right\},
\end{array}\right. \\
& \mathbb{Y}_{\delta}^{-}=\left\{S=\left(S_{1}, \ldots, S_{k}\right) \in \mathbb{Y}: d\left(S_{*}^{-}, S_{1}\right) \geq \delta\right. \text { and } \\
& \left.d\left(S_{i}, S_{i+1}\right) \geq \eta_{i}^{-} d\left(S_{i-1}, S_{i+1}\right) \text { for } i=1, \ldots, k\right\} .
\end{aligned}
$$

5.9. Remarks. (a) Observe that if the multisurfaces $S^{ \pm}:=\left(S_{1}^{ \pm}, S_{2}^{ \pm}, \ldots, S_{k}^{ \pm}\right) \in \mathbb{Y}$ are chosen such that

$$
d\left(S_{1}^{-}, S_{*}^{-}\right) \geq \delta, d\left(S_{k}^{+}, S_{*}^{+}\right) \geq \delta \quad \text { and } \quad d\left(S_{i}^{ \pm}, S_{i \mp 1}^{ \pm}\right) \geq \eta_{i}^{ \pm} d\left(S_{i}^{ \pm}, S_{i \pm 1}^{ \pm}\right)
$$

for $i=1, \ldots, k$, then $d\left(S_{i}^{ \pm}, S_{i \pm 1}^{ \pm}\right) \geq \delta_{i}^{ \pm}$for $i=1, \ldots, k$. (b) It follows from Part (a) that for each sufficiently small $\delta>0$, there exists a value $C=C(\delta)>0$ such that $d\left(S_{i}, S_{i+1}\right) \geq C$ for $i=0, \ldots, k$, uniformly for all multisurfaces $S \in \mathbb{Y}_{\delta}^{ \pm}$.

5.10. Lemma. Given the fixed values $\delta>0$ and $\varepsilon \in\left(0, \tilde{\varepsilon}_{0}(\delta)\right]$, let $S_{n}^{ \pm}:=\left(S_{n, 1}^{ \pm}, S_{n, 2}^{ \pm}\right.$, $\left.\ldots, S_{n, k}^{ \pm}\right)$denote a multisurface in $\mathfrak{S}_{\varepsilon}^{ \pm}\left(S_{*}^{-}, S_{*}^{+}\right) \cap \mathbb{Y}_{\delta}^{ \pm}$. Then there exists at least one multisurface $S_{n+1}^{ \pm}=\left(S_{n+1,1}^{ \pm}, S_{n+1,2}^{ \pm}, \ldots, S_{n+1, k}^{ \pm}\right) \in \mathbb{Y}\left(S_{*}^{-}, S_{*}^{+}\right)$such that for each $i=1, \ldots, k$, we have (a) $S_{n+1, i}^{ \pm} \in \mathfrak{S}_{\varepsilon, i}\left(S_{n, i-1}^{ \pm}, S_{n, i+1}^{ \pm}\right)$, (b) $S_{n+1, i}^{+} \leq S_{n, i}^{+}$, (c) $S_{n+1, i}^{-} \geq S_{n, i}^{-}$, and (d) $d\left(S_{n+1, i}^{-}, S_{n, i+1}^{-}\right) \geq \eta_{i}^{-} d\left(S_{n, i-1}^{-}, S_{n, i+1}^{-}\right)$. It follows from these properties that $S_{n+1}^{ \pm} \in \mathfrak{S}_{\varepsilon}^{ \pm}\left(S_{*}^{-}, S_{*}^{+}\right) \cap \mathbb{Y}_{\delta}^{ \pm}$.

Proof. For each $i=1, \ldots, k$, the existence of a surface $S_{n+1, i}^{+} \in \mathfrak{S}_{\varepsilon, i}\left(S_{n, i-1}^{+}, S_{n, i+1}^{+}\right)$ with property (b) follows from Corollary 4.5, whereas the existence of a surface $S_{n+1, i}^{-} \in \mathfrak{S}_{\varepsilon, i}\left(S_{n, i-1}^{-}, S_{n, i+1}^{-}\right)$with properties (c) and (d) follows from Corollary 4.7. Clearly

$$
\begin{gathered}
S_{n+1, i-1}^{+} \leq S_{n, i-1}^{+}<S_{n+1, i}^{+} \leq S_{n, i}^{+}<S_{n+1, i+1}^{+} \leq S_{n, i+1}^{+}, \\
S_{n, i-1}^{-} \leq S_{n+1, i}^{-}<S_{n, i}^{-} \leq S_{n+1, i}^{-}<S_{n, i+1}^{-} \leq S_{n+1, i+1}^{-}
\end{gathered}
$$

for $i=1, \ldots, k$. In view of (5.1) and (5.2), it follows from Lemma 3.11 that

$$
S_{n+1, i}^{ \pm} \in \mathfrak{S}_{\varepsilon, i}^{ \pm}\left(S_{n+1, i-1}^{ \pm}, S_{n+1, i+1}^{ \pm}\right)
$$

for $i=1, \ldots, k$. Thus, $S_{n+1}^{ \pm} \in \mathfrak{S}_{\varepsilon}^{ \pm}\left(S_{*}^{-}, S_{*}^{+}\right)$. Now Lemma 4.4 implies that

$$
d\left(S_{n+1, i-1}^{+}, S_{n+1, i}^{+}\right) \geq \eta_{i}^{+} d\left(S_{n+1, i-1}^{+}, S_{n+1, i+1}^{+}\right)>\eta_{i}^{+} d\left(S_{n+1, i}^{+}, S_{n+1, i+1}^{+}\right)
$$


for $i=1, \ldots, k$. Since $d\left(S_{n+1, k}^{+}, S_{*}^{+}\right) \geq d\left(S_{n, k}^{+}, S_{*}^{+}\right) \geq \delta$ (due to (5.1)), it follows from (5.4) that $S_{n+1}^{+} \in \mathbb{Y}_{\delta}^{+}$. We also have

$$
\begin{aligned}
d\left(S_{n+1, i}^{-}, S_{n+1, i+1}^{-}\right) & \geq d\left(S_{n+1, i}^{-}, S_{n, i+1}^{-}\right) \\
& \geq \eta_{i}^{-} d\left(S_{n, i-1}^{-}, S_{n, i+1}^{-}\right) \geq \eta_{i}^{-} d\left(S_{n+1, i-1}^{-}, S_{n+1, i}^{-}\right)
\end{aligned}
$$

for $i=1, \ldots, k$, due to (5.2) and property (d), while also

$$
d\left(S_{*}^{-}, S_{n+1,1}^{-}\right) \geq d\left(S_{*}^{-}, S_{n, 1}^{-}\right) \geq \delta,
$$

due to (5.2) and the fact that $S_{n}^{-} \in \mathbb{Y}_{\delta}^{-}$. It follows from (5.5), (5.6), and Remark 5.9 (a) that for each $i=1, \ldots, k$, we have

$$
\begin{aligned}
& d\left(S_{n+1, i-1}^{-}, S_{n+1, i+1}^{-}\right) \geq d\left(S_{n+1, i-1}^{-}, S_{n+1, i}^{-}\right) \geq \delta_{i}^{-}, \\
& d\left(S_{n+1, i}^{-}, S_{n+1, i+1}^{-}\right) \geq \delta_{i+1}^{-}=\delta_{i}^{-} \eta_{i}^{-}=\delta_{i}^{-} h_{i}\left(\delta_{i}^{-} / 2\right) .
\end{aligned}
$$

In view of (5.7) and (5.8), it follows from Lemma 4.6 that

$$
d\left(S_{n+1, i}^{-}, S_{n+1, i+1}^{-}\right) \geq \eta_{i}^{-} d\left(S_{n+1, i-1}^{-}, S_{n+1, i+1}^{-}\right)
$$

for $i=1, \ldots, k$. Then (5.6) and (5.9) imply that $S_{n+1}^{-} \in \mathbb{Y}_{\delta}^{-}$.

5.11. Proof of Theorem 5.5. Choose $\delta>0$ so small that $S_{1}^{ \pm} \in \mathbb{Y}_{\delta}^{ \pm}$, where the multisurfaces $S_{1}^{ \pm}$were introduced in Lemma 5.7. For each sufficiently small $\varepsilon>0$, let the monotone sequences $\left(S_{\varepsilon, n}^{ \pm}\right)_{n=1}^{\infty}$ (with $S_{\varepsilon, 1}^{ \pm}:=S_{1}^{ \pm}$) be defined recursively by Lemma 5.10. Then for sufficiently small $\delta>0$, and for any $\varepsilon \in\left(0, \tilde{\varepsilon}_{0}(\delta)\right]$, we have $S_{\varepsilon, n}^{ \pm} \in \mathfrak{S}_{\varepsilon}^{ \pm}\left(S_{*}^{-}, S_{*}^{+}\right) \cap \mathbb{Y}_{\delta}^{ \pm}$for all $n \in \mathbb{N}$. Therefore $d\left(S_{\varepsilon, n, i}^{ \pm}, S_{\varepsilon, n, i+1}^{ \pm}\right) \geq C>$ 0 uniformly for $i=0, \ldots, k, n \in \mathbb{N}$, and sufficiently small $\varepsilon$ (by Remarks 5.9 ), where we use the component-wise representation $S_{\varepsilon, n}^{ \pm}=\left(S_{\varepsilon, n, 1}^{ \pm}, S_{\varepsilon, n, 2}^{ \pm}, \ldots, S_{\varepsilon, n, k}^{ \pm}\right)$. For fixed $\varepsilon>0$ and fixed $i=1, \ldots, k$, it follows from Lemma 5.10(b), (c) that $\left(S_{\varepsilon, n, i}^{-}\right)_{n=1}^{\infty}\left(\operatorname{resp} .\left(S_{\varepsilon, n, i}^{+}\right)_{n=1}^{\infty}\right)$ is a weakly increasing (decreasing) sequence of convex surfaces such that $S_{\varepsilon, n, i}^{-}<S_{*}^{+}$(resp. $S_{\varepsilon, n, i}^{+}>S_{*}^{-}$). Therefore, for each sufficiently small $\varepsilon>0$ and for each $i=1, \ldots, k$, there exists a surface $S_{\varepsilon, i}^{ \pm} \in \mathbb{X}$ such that $\Delta\left(S_{\varepsilon, n, i}^{ \pm}, S_{\varepsilon, i}^{ \pm}\right) \rightarrow 0$ as $n \rightarrow \infty$. It easily follows by continuity that $d\left(S_{\varepsilon, i}^{ \pm}, S_{\varepsilon, i+1}^{ \pm}\right) \geq$ $C>0$ for $i=0, \ldots, k$. Moreover,

$$
\begin{aligned}
S_{\varepsilon, i}^{ \pm} & =\lim _{n \rightarrow \infty} S_{\varepsilon, n+1, i}^{ \pm}=\lim _{n \rightarrow \infty} T_{\varepsilon, i}\left(S_{\varepsilon, n, i-1}^{ \pm}, S_{\varepsilon, n+1, i}^{ \pm}, S_{\varepsilon, n, i+1}^{ \pm}\right) \\
& =T_{\varepsilon, i}\left(S_{\varepsilon, i-1}^{ \pm}, S_{\varepsilon, i}^{ \pm}, S_{\varepsilon, i+1}^{ \pm}\right)
\end{aligned}
$$

for $i=1, \ldots, k$, due to Lemma 3.8 and the fact that $S_{\varepsilon, n+1, i}^{ \pm} \in \mathfrak{S}_{\varepsilon}\left(S_{\varepsilon, n, i-1}^{ \pm}, S_{\varepsilon, n, i+1}^{ \pm}\right)$ (see Lemma 5.10(a)). Therefore $S_{\varepsilon, i}^{ \pm} \in \mathfrak{S}_{\varepsilon, i}\left(S_{\varepsilon, i-1}^{ \pm}, S_{\varepsilon, i+1}^{ \pm}\right)$for $i=1, \ldots, k$, whence $S_{\varepsilon}^{ \pm}:=\lim _{n \rightarrow \infty} S_{\varepsilon, n}^{ \pm} \in \mathfrak{S}_{\varepsilon}\left(S_{*}^{-}, S_{*}^{+}\right)$.

5.12. Proof of Theorem 2.11(a). Let a positive null sequence $\left(\varepsilon_{n}\right)_{n=1}^{\infty}$ be given such that $\varepsilon_{n} \in\left(0, \tilde{\varepsilon}_{0}\right]$ for all $n \in \mathbb{N}$. For each $n \in \mathbb{N}$, let $S_{n}=\left(S_{n, 1}, \ldots, S_{n, k}\right) \in \mathbb{Y}$ denote a solution of Problem 5.2 at the value $\varepsilon=\varepsilon_{n}$ such that $d\left(S_{n, i}, S_{n, i+1}\right) \geq$ $C>0$ for all $i=0, \ldots, k$ (this exists due to Theorem 5.5). Also, for each $n$ and $i$, the surface $S_{n, i}$ is starlike relative to all points in some fixed ball $B_{\delta}(0) \subset D_{*}^{-}$. Therefore, the family of surfaces $\left\{S_{n, i}: n \in \mathbb{N}, i=1, \ldots, k\right\}$ corresponds to a family of polar-coordinate representations (relative to any fixed origin located inside $D_{*}^{-}$) which is equicontinuous and uniformly bounded. Applying the theorem of AscoliArzela, we pass to a subsequence (still denoted by $\left(S_{n}\right)_{n=1}^{\infty}$ ) such that $S_{n, i} \rightarrow \widetilde{S}_{i} \in \mathbb{X}$ as $n \rightarrow \infty$ for each $i=1, \ldots, k$. It easily follows from the properties of the 
multisurfaces $S_{n}, n \in \mathbb{N}$, that $\widetilde{S}:=\left(\widetilde{S}_{1}, \ldots, \widetilde{S}_{k}\right) \in \mathbb{Y}$ and $d\left(\widetilde{S}_{i}, \widetilde{S}_{i+1}\right) \geq C>0$ for $i=0, \ldots, k$. By definition, $\widetilde{S}$ is the weak solution of Problem 1.1.

5.13. Theorem. If $\widetilde{S}:=\left(\widetilde{S}_{1}, \ldots, \widetilde{S}_{k}\right) \in \mathbb{Y}$ is a weak solution of Problem 1.1, then for each $i=1, \ldots, k$, the surface $\widetilde{S}_{i} \in \mathbb{X}$ is a weak solution of Problem 2.1 (in the sense of Definition 3.12) in the particular case where one defines $S_{*}^{-}=\widetilde{S}_{i-1}, S_{*}^{+}=$ $\widetilde{S}_{i+1}$ and $F=F_{i}$.

5.14. Remark. To prove Theorem 2.11(b), we must show that weak solutions of Problem 1.1 are in fact classical solutions of Problem 1.1. However, in view of Theorem 5.13, the regularity properties of weak solutions of Problem 1.1 follow from the same regularity properties of weak solutions of Problem 2.1.

5.15. Theorem. Let $\left(S_{*, n}^{ \pm}\right)_{n=1}^{\infty}$ and $\left(\widetilde{S}_{n}\right)_{n=1}^{\infty}$ be sequences such that for each $n \in \mathbb{N}$, $\widetilde{S}_{n}=\left(\widetilde{S}_{1, n}, \ldots, \widetilde{S}_{k, n}\right)$ is a weak solution of Problem 1.1 corresponding to the surfaces $S_{*, n}^{ \pm} \in \mathbb{X}\left(\right.$ with $\left.S_{*, n}^{-}<S_{*, n}^{+}\right)$. Suppose that $S_{*, n}^{ \pm} \rightarrow S_{*}^{ \pm} \in \mathbb{X}$ and $\widetilde{S}_{n} \rightarrow \widetilde{S} \in$ $\mathbb{Y}\left(S_{*}^{-}, S_{*}^{+}\right)$, both as $n \rightarrow \infty$. Then $\widetilde{S}$ is a weak solution of Problem 1.1 .

Proof. For each $n \in \mathbb{N}$, there exist sequences $\left(\varepsilon_{n, m}\right)_{m=1}^{\infty},\left(S_{*, n, m}^{ \pm}\right)_{m=1}^{\infty},\left(S_{n, m}\right)_{m=1}^{\infty}$ such that $\varepsilon_{n, m} \downarrow 0, S_{*, n, m}^{ \pm} \rightarrow S_{*, n}^{ \pm}$, and $S_{n, m} \rightarrow \widetilde{S}_{n}$ as $m \rightarrow \infty$, and such that $S_{n, m} \in \mathfrak{S}\left(S_{*, n, m}^{-}, S_{*, n, m}^{+} ; \varepsilon_{n, m}\right)$ for each $m \in \mathbb{N}$. For each $n \in \mathbb{N}$, we define $\hat{\varepsilon}_{n}=$ $\varepsilon_{n, m(n)}, \widehat{S}_{*, n}^{ \pm}=S_{*, n, m(n)}^{ \pm}$, and $\widehat{S}_{n}=S_{n, m(n)}$, where $m(n)$ is chosen so large that $\varepsilon_{n, m(n)}<(1 / n), \Delta\left(S_{*, n, m(n)}^{ \pm}, S_{*}^{ \pm}\right)<2 \Delta\left(S_{*, n}^{ \pm}, S_{*}^{ \pm}\right)$, and $\Delta\left(S_{n, m(n)}, \widetilde{S}\right)<2 \Delta\left(\widetilde{S}_{n}, \widetilde{S}\right)$. Then $\widehat{S}_{n} \rightarrow \widetilde{S}$ and $\widehat{S}_{*, n}^{ \pm} \rightarrow S_{*}^{ \pm}$as $n \rightarrow \infty$, and $\widehat{S}_{n} \in \mathfrak{S}\left(\widehat{S}_{*, n}^{-}, \widehat{S}_{*, n}^{+} ; \hat{\varepsilon}_{n}\right)$ for all $n \in \mathbb{N}$.

\section{Preliminary Regularity properties of Weak solutions of Problem 2.1}

6.1. Notational conventions. Throughout the remainder of this paper, $\widetilde{S}$ denotes a given weak solution of Problem 2.1 (in the sense of Definition 3.12), and $\left(S_{*, n}^{-}, S_{n}, S_{*, n}^{+}\right)_{n=1}^{\infty}$ denotes an approximating sequence corresponding to a positive null sequence $\left(\varepsilon_{n}\right)_{n=1}^{\infty}$. We define $\widetilde{U}^{ \pm}(x)=U^{ \pm}\left(S_{*}^{ \pm}, \widetilde{S} ; x\right)$ and $\widetilde{Q}^{ \pm}(x)=$ $\left|\nabla \widetilde{U}^{ \pm}(x)\right|$ both in $\widetilde{\Omega}^{ \pm}:=\Omega\left(S_{*}^{ \pm}, \widetilde{S}\right)$, and $\widetilde{U}_{n}^{ \pm}(x)=U^{ \pm}\left(S_{*, n}^{ \pm}, S_{n} ; x\right)$ and $Q_{n}^{ \pm}(x)=$ $\left|\nabla U_{n}^{ \pm}(x)\right|$ both in $\Omega_{*, n}^{ \pm}:=\Omega\left(S_{*, n}^{ \pm}, S_{n}\right)$. For $n \in \mathbb{N}$ and $\alpha \in(0,1)$, we also define $\Omega_{*, n}=\Omega\left(S_{*, n}^{-}, S_{*, n}^{+}\right), \widetilde{S}_{\alpha}^{ \pm}=\left\{\widetilde{U}^{ \pm}(x)=\alpha\right\}, S_{n}^{ \pm}=\left\{U_{n}^{ \pm}(x)=\varepsilon_{n}\right\}, \Omega_{n}^{ \pm}=\Omega\left(S_{n}, S_{n}^{ \pm}\right)$, $S_{n, \alpha}^{ \pm}=\left\{U_{n}^{ \pm}(x)=\alpha \varepsilon_{n}\right\}, \Omega_{n, \alpha}^{ \pm}=\Omega\left(S_{n}, S_{n, \alpha}^{ \pm}\right), \widehat{S}_{n, \alpha}^{ \pm}=\left\{U_{n}^{ \pm}(x)=\alpha\right\}$, and $\widehat{\Omega}_{n, \alpha}^{ \pm}=$ $\Omega\left(S_{n}, \widehat{S}_{n, \alpha}^{ \pm}\right)$. Also, $\tilde{\nu}(x)$ denotes the exterior normal to the level surface of $\widetilde{U}^{ \pm}$ through the point $x \in \Omega_{*}$, and $\nu_{n}(x)$ denotes the exterior normal to the level surface of $U_{n}^{ \pm}$through the point $x \in \Omega_{*, n} \backslash S_{n}$ (thus $\tilde{\nu}(x)= \pm\left(\nabla \widetilde{U}^{ \pm}(x) / \widetilde{Q}^{ \pm}(x)\right)$ in $\widetilde{\Omega}^{ \pm}$ and $\nu_{n}(x)= \pm\left(\nabla U_{n}^{ \pm}(x) / Q_{n}^{ \pm}(x)\right)$ in $\left.\Omega_{*, n}^{ \pm}\right)$.

6.2. Theorem. Under Assumptions 2.2(a)-(d), any (convex) weak solution $\widetilde{S}$ of Problem 2.1 has the following properties: (a) We have that $\mathfrak{C}_{0} \leq \widetilde{Q}^{ \pm}(x) \leq \mathfrak{C}_{1}$ in $\left\{x \in \widetilde{\Omega}^{ \pm}: d\left(x, S_{*}^{ \pm}\right) \geq d_{0}\right\}$, where $d_{0}=(1 / 3) \min \left\{d\left(\widetilde{S}, S_{*}^{-}\right), d\left(\widetilde{\widetilde{S}}, S_{*}^{+}\right)\right\}$. (b) $\widetilde{S}$ is a uniformly $C^{1}$-surface. (c) The functions $\widetilde{U}^{ \pm}(x)$ are differentiable on $\widetilde{S}$ in the following sense: For any fixed $x_{0} \in \widetilde{S}$, there exist positive values $\lambda_{0}^{ \pm}=\lambda^{ \pm}\left(x_{0}\right) \in$ $\left[\mathfrak{C}_{0}, \mathfrak{C}_{1}\right]$ such that

$$
\widetilde{U}^{ \pm}(x)= \pm \lambda_{0}^{ \pm} \tilde{\nu}_{0} \cdot\left(x-x_{0}\right)+o\left(\left|x-x_{0}\right|\right)
$$


as $x \rightarrow x_{0}$ relative to $\widetilde{\Omega}^{ \pm}$, where $\tilde{\nu}_{0}=\tilde{\nu}\left(x_{0}\right)=$ the exterior normal to $\widetilde{S}$ at $x_{0}$. We define the derivatives at $x_{0} \in \widetilde{S}$ by $\nabla \widetilde{U}^{ \pm}\left(x_{0}\right)= \pm \lambda_{0}^{ \pm} \tilde{\nu}_{0}$. (d) Let $\widetilde{Q}^{ \pm}(x)=$ $\left|\nabla \widetilde{U}^{ \pm}(x)\right|=\lambda^{ \pm}(x)$ for $x \in \widetilde{S}$. Then the function $\pm \widetilde{Q}^{ \pm}(x): \widetilde{S} \rightarrow \mathbb{R}$ is lower semicontinuous in the sense that

$$
\pm \widetilde{Q}^{ \pm}\left(x_{0}\right) \leq \liminf _{x \rightarrow x_{0}}\left( \pm \widetilde{Q}^{ \pm}(x)\right),
$$

(e) For any fixed $x_{0} \in \widetilde{S}$ and constant $0<C<1$, we have $\left|\nabla \widetilde{U}^{ \pm}(x)-\nabla \widetilde{U}^{ \pm}\left(x_{0}\right)\right| \leq$ $z\left(x_{0}, \varepsilon\right)$ uniformly in $\left\{x \in \widetilde{\Omega}^{ \pm} \cap B_{\varepsilon}\left(x_{0}\right): d(x, \widetilde{S}) \geq C \varepsilon\right\}$, where $z\left(x_{0}, \varepsilon\right) \rightarrow 0$ as $\varepsilon \downarrow 0$. (f) For any $x_{0} \in \widetilde{S}$, we have $\widetilde{Q}^{+}(x) \uparrow \widetilde{Q}^{+}\left(x_{0}\right)$ and $\widetilde{Q}^{-}(x) \downarrow \widetilde{Q}^{-}\left(x_{0}\right)$ as $x \rightarrow x_{0}$ along a curve of steepest ascent of the function $\widetilde{U}^{ \pm}(\cdot)$.

Proof. Part (a) is due to Corollary 4.9. The proofs of Parts (b), (c), and (e) (based on Part (a)) are due to Caffarelli and Spruck [15], Theorem 4.12. Part (f) follows from Part (e), Lemma 3.4(d), and Lemma 6.4. Finally, the proof of Part (d) (based on Part (f)) is given in [9], Lemma 5.2.

6.3. Lemma. Let $S$ denote any fixed uniformly $C^{1}$-surface in $\mathbb{X}$, and let $\left(S_{n}\right)_{n=1}^{\infty}$ denote any sequence of surfaces in $\mathbb{X}$ such that $\Delta\left(S_{n}, S\right) \rightarrow 0$ as $n \rightarrow \infty$. Let $\nu(x)$ be the exterior normal to $S$ at $x \in S$, and, for each $n \in \mathbb{N}$ and $x \in S_{n}$, choose $\nu_{n}(x)$ such that $\nu_{n}(x) \cdot(y-x) \leq 0$ for all $y \in D\left(S_{n}\right)$. Then there exists a null function $z(t, n): \mathbb{R}_{+} \times \mathbb{N} \rightarrow \mathbb{R}$ such that

$$
\left|\nu_{n}(x)-\nu\left(x_{0}\right)\right| \leq z\left(\left|x-x_{0}\right|, n\right)
$$

uniformly for all $x_{0} \in S, x \in S_{n}$, and $n \in \mathbb{N}$.

Proof. According to [9], Lemma 3.4, there exists a null function $\zeta_{0}(n)$ such that $\left|\nu_{n}\left(x_{n}\right)-\nu\left(\hat{x}_{n}\right)\right| \leq \zeta_{0}(n)$ for any point $x_{n} \in S_{n}$, where $\hat{x}_{n} \in S$ is chosen such that $\left|\hat{x}_{n}-x_{n}\right|=d\left(S, x_{n}\right)$. Thus

$$
\begin{aligned}
\left|\nu_{n}\left(x_{n}\right)-\nu\left(x_{0}\right)\right| & \leq\left|\nu_{n}\left(x_{n}\right)-\nu\left(\hat{x}_{n}\right)\right|+\left|\nu\left(\hat{x}_{n}\right)-\nu\left(x_{0}\right)\right| \leq \zeta_{0}(n)+z\left(\left|\hat{x}_{n}-x_{0}\right|\right) \\
& \leq \zeta_{0}(n)+z\left(\left|\hat{x}_{n}-x_{n}\right|+\left|x_{n}-x_{0}\right|\right),
\end{aligned}
$$

where $\left|\hat{x}_{n}-x_{n}\right| \leq \zeta_{1}(n):=C \Delta\left(S_{n}, S\right)$ for some constant $C$ (independent of $x_{n} \in$ $\left.S_{n}\right)$.

6.4. Lemma. Let $S \in \mathbb{X}$ be a uniformly $C^{1}$-surface. For an $x \in \Omega_{*}$, let $\nu(x)$ denote the unit exterior normal to the level surface of $U^{ \pm}$through $x$, where $U^{ \pm}(x):=$ $U^{ \pm}\left(S_{*}^{ \pm}, S ; x\right)$. Then for any given compact subset $K$ of $\Omega_{*}$, there exists a null function $z(t)$ such that

$$
|\nu(x)-\nu(y)| \leq z(|x-y|)
$$

for all $x, y \in K$.

Proof. Assume the assertion is not true. Then there exist sequences of points $x_{n}, y_{n} \in K, n \in \mathbb{N}$, such that $\left|x_{n}-y_{n}\right| \rightarrow 0$ as $n \rightarrow \infty$, while $\left|\nu\left(x_{n}\right)-\nu\left(y_{n}\right)\right| \geq \rho_{0}>0$ for all $n \in \mathbb{N}$. By passing to a subsequence (still indexed by $n \in \mathbb{N}$ ), we can assume $x_{n}, y_{n} \rightarrow x_{0} \in K$ as $n \rightarrow \infty$. Clearly $x_{0} \in S$, since $\nu(x)$ is continuous in $\Omega_{*} \backslash S$. Then $x_{n} \in S_{n}^{\prime}$ and $y_{n} \in S_{n}^{\prime \prime}$, where $S_{n}^{\prime}, S_{n}^{\prime \prime}$ denote level surfaces of the functions $U^{ \pm}(x)$ such that $\Delta\left(S, S_{n}^{\prime}\right), \Delta\left(S, S_{n}^{\prime \prime}\right) \rightarrow 0$ as $n \rightarrow \infty$. Therefore, $\left|\nu\left(x_{n}\right)-\nu\left(x_{0}\right)\right| \leq z\left(\left|x_{n}-x_{0}\right|, n\right)$ and $\left|\nu\left(y_{n}\right)-\nu\left(x_{0}\right)\right| \leq z\left(\left|y_{n}-x_{0}\right|, n\right)$ for all $n \in \mathbb{N}$, 
by Lemma 6.3. Therefore $\left|\nu\left(x_{n}\right)-\nu\left(y_{n}\right)\right| \rightarrow 0$ as $n \rightarrow \infty$, a contradiction which proves the assertion.

6.5. Lemma. Let $\widetilde{S} \in \mathbb{X}$ be a weak solution of Problem 2.1, and $\left(S_{*, n}^{-}, S_{n}, S_{*, n}^{+}\right)_{n=1}^{\infty}$ a corresponding approximating sequence. If $\widetilde{S}$ is a uniformly $C^{1}$-surface, then for any compact subset $K$ of $\Omega_{*}$, we have (using notation from $\S 6.1$ ):

$$
\sup \left\{\left|\nu_{n}(x)-\tilde{\nu}(x)\right|: x \in K \backslash S_{n}\right\} \rightarrow 0 \text { as } n \rightarrow \infty .
$$

Proof. Since $U_{\tilde{n}}^{ \pm}(x) \rightarrow \widetilde{U}^{ \pm}(x)$ relative to compact subsets of $\widetilde{\Omega}^{ \pm}$, we have $\nabla U_{n}^{ \pm}(x)$ $\rightarrow \nabla \widetilde{U}^{ \pm}(x)$ in $\widetilde{\Omega}^{ \pm}$as $n \rightarrow \infty$. Since $\widetilde{Q}^{ \pm}(x)>0$ in $\widetilde{\Omega}^{ \pm}$, it follows that $\nu_{n}(x) \rightarrow \tilde{\nu}(x)$ uniformly in compact subsets of $\widetilde{\Omega}^{ \pm}$. Therefore, if the assertion is false, then there exist a value $\rho_{0}>0$ and a sequence $\left(x_{n}\right)$ such that $x_{n} \in K \backslash S_{n}$ and $\mid \nu_{n}\left(x_{n}\right)-$ $\tilde{\nu}\left(x_{n}\right) \mid \geq \rho_{0}$ for all $n \in \mathbb{N}$, and such that $d\left(x_{n}, \widetilde{S}\right) \rightarrow 0$ as $n \rightarrow \infty$. By passing to a subsequence (still indexed by $n \in \mathbb{N}$ ), we can assume that $x_{n} \rightarrow x_{0} \in \widetilde{S}$ as $n \rightarrow \infty$. Therefore, we have $x_{n} \in S_{n}^{\prime}$ for $n \in \mathbb{N}$, where (a) $S_{n}^{\prime}$ denotes a level surface of $U_{n}^{+}$or $U_{n}^{-}$for each $n \in \mathbb{N}$, and (b) $\Delta\left(\widetilde{S}, S_{n}^{\prime}\right) \rightarrow 0$ as $n \rightarrow \infty$. For $n \rightarrow \infty$, we have $\nu_{n}\left(x_{n}\right) \rightarrow \tilde{\nu}\left(x_{0}\right)$ by Lemma 6.3 , and $\tilde{\nu}\left(x_{n}\right) \rightarrow \tilde{\nu}\left(x_{0}\right)$ by Lemma 6.4 . This contradiction proves the assertion.

6.6. Lemma. Let $\widetilde{S} \in \mathbb{X}$ be a weak solution of Problem 2.1, let $\left(S_{*, n}^{-}, S_{n}, S_{*, n}^{+}\right)_{n=1}^{\infty}$ be a corresponding approximating sequence. For each $n \in \mathbb{N}$, let $M_{n}^{ \pm}$denote the set of all pairs $(x, y)($ with $x \neq y)$ such that (a) $x$ and $y$ are both in the closure of $\Omega_{n}^{ \pm}:=\Omega\left(S_{n}, S_{n}^{ \pm}\right)$, and (b) $x$ and $y$ are the endpoints of an arc of steepest ascent $\gamma_{n}^{ \pm}(x, y)$ of the function $U_{n}^{ \pm}(\cdot)$. If $\widetilde{S}$ is a uniformly $C^{1}$-surface, then

$$
\begin{gathered}
\lim _{n \rightarrow \infty} \sup \left\{\left|\gamma_{n}^{ \pm}(x, y)\right|:(x, y) \in M_{n}^{ \pm}\right\}=0, \\
\lim _{n \rightarrow \infty} \sup \left\{\left(\left|\gamma_{n}^{ \pm}(x, y)\right| /|x-y|\right):(x, y) \in M_{n}^{ \pm}\right\}=1,
\end{gathered}
$$

where $\left|\gamma_{n}^{ \pm}(x, y)\right|$ denotes the arc-length of $\gamma_{n}^{ \pm}(x, y)$.

Proof sketch. For given $\kappa>1$, it follows from Lemmas 3.5, 4.8, 6.4, and 6.5 that there exist positive null sequences $\left(\alpha_{n}\right)_{n=1}^{\infty}$ and $\left(\beta_{n}\right)_{n=1}^{\infty}$ such that

$$
B\left(x_{0} ; \alpha_{n}\right) \cap S_{n} \neq \varnothing ; \quad B\left(x_{0} ; \alpha_{n}\right) \cap S_{n}^{ \pm} \neq \varnothing ; \quad B\left(y ; \alpha_{n}\right) \cap \widetilde{S} \neq \varnothing
$$

for any $x_{0} \in \widetilde{S}, n \in \mathbb{N}$, and $y \in S_{n}$, and such that

$$
\left|\nu_{n}(x)-\tilde{\nu}\left(x_{0}\right)\right| \leq \beta_{n}
$$

for any $x_{0} \in \widetilde{S}, x \in B\left(x_{0} ; \kappa \alpha_{n}\right)$, and $n \in \mathbb{N}$. For each $x_{0} \in \widetilde{S}$, let $R_{n}\left(x_{0}\right)$ (resp. $\left.R_{n}^{\perp}\left(x_{0}\right)\right)$ denote the set of all points $y \in C l\left(B\left(x_{0} ; \kappa \alpha_{n}\right)\right)$ having the property that $\left|(y-x) \cdot \tilde{\nu}\left(x_{0}\right)\right| \geq|y-x| \cos \left(\beta_{n}\right)\left(\operatorname{resp} .\left|(y-x) \cdot \tilde{\nu}\left(x_{0}\right)\right| \leq|y-x| \sin \left(\beta_{n}\right)\right)$ for at least one corresponding point $x=x(y) \in C l\left(B\left(x_{0} ; \alpha_{n}\right)\right)$. It follows from $(6.3 \mathrm{a}, \mathrm{b})$ and $(6.4)$ that for any $x_{0} \in \widetilde{S}$, and for sufficiently large $n \in \mathbb{N}$ (independent of $x_{0} \in \widetilde{S}$ ), the domains $\Omega_{n}^{ \pm} \cap B\left(x_{0} ; \kappa \alpha_{n}\right)$ are both simply connected and are contained in $R_{n}^{\perp}\left(x_{0}\right)$, and any admissible arc $\gamma_{n}^{ \pm}(x, y)$ joining $x \in B\left(x_{0} ; \alpha_{n}\right) \cap S_{n}$ to $y \in S_{n}^{ \pm}$must be such that $\gamma_{n}^{ \pm}(x, y) \subset R_{n}\left(x_{0}\right) \cap R_{n}^{\perp}\left(x_{0}\right) \subset B\left(x_{0} ; \kappa \alpha_{n}\right)$. Therefore, in view of (6.4), we have that $\left|\nu_{n}(z)-\nu_{n}\left(z^{\prime}\right)\right| \leq 2 \beta_{n}$, uniformly for all sufficiently large $n \in \mathbb{N}$, all $x_{0} \in \widetilde{S}$, all admissible $\operatorname{arcs} \gamma_{n}^{ \pm}(x, y)$ joining $x \in B\left(x_{0} ; \alpha_{n}\right) \cap S_{n}$ to $y \in S_{n}^{ \pm}$, and all pairs of points $z, z^{\prime} \in \gamma_{n}^{ \pm}(x, y)$. Therefore, there exists a sequence $\left(\rho_{n}\right)$ such that $\rho_{n} \downarrow 1$ as $n \rightarrow \infty$, and such that $\left(\left|\gamma_{n}^{ \pm}(x, y)\right| /|x-y|\right) \leq \rho_{n}$ in the same uniform 
sense. On the other hand, for every $n \in \mathbb{N}$ and $x \in S_{n}$, we have $x \in B\left(x_{0} ; \alpha_{n}\right)$ for some $x_{0} \in \widetilde{S}$, by (6.3c). The assertions (6.1), (6.2) follow.

6.7. Lemma. Let $\widetilde{S} \in \mathbb{X}$ be a weak solution of Problem 2.1, and $\left(S_{*, n}^{-}, S_{n}, S_{*, n}^{+}\right)_{n=1}^{\infty}$ a corresponding approximating sequence. Let the sequences $\left(\tilde{x}_{n}\right)_{n=1}^{\infty}$ and $\left(x_{n}^{ \pm}\right)_{n=1}^{\infty}$ be given such that $\tilde{x}_{n} \in \widetilde{S}$ and $x_{n}^{ \pm} \in \Omega_{n}^{ \pm} \cap \widetilde{\Omega}^{\mp}$ for all $n \in \mathbb{N}$, and $\left(x_{n}^{ \pm}-\tilde{x}_{n}\right) \rightarrow 0$ as $n \rightarrow \infty$. For each $n \in \mathbb{N}$, let $\gamma_{n}^{ \pm}$denote the arc of steepest ascent of $U_{n}^{ \pm}(\cdot)$ which joins its initial point $x_{n}^{ \pm}$to the surface $S_{*, n}^{ \pm}$. Then there exists a sequence $\left(\hat{x}_{n}^{ \pm}\right)_{n=1}^{\infty}$ such that $\hat{x}_{n}^{ \pm} \in \widetilde{\Omega}^{ \pm} \cap \gamma_{n}^{ \pm}$for all $n \in \mathbb{N}$, and such that $\left(\hat{x}_{n}^{ \pm}-\tilde{x}_{n}\right) \rightarrow 0$ as $n \rightarrow \infty$.

Proof. As in the proof of Lemma 6.6, there exist, for given $\kappa>1$, positive null sequences $\left(\alpha_{n}\right)_{n=1}^{\infty},\left(\beta_{n}\right)_{n=1}^{\infty}$ such that for each $n \in \mathbb{N}$, we have $x_{n}^{ \pm} \in B\left(\tilde{x}_{n} ; \alpha_{n}\right)$ and $\left|\nu_{n}(x)-\tilde{\nu}\left(\tilde{x}_{n}\right)\right| \leq \beta$ for all $x \in B\left(\tilde{x}_{n} ; \kappa \alpha_{n}\right)$. Therefore, $\gamma_{n}^{ \pm} \cap \widetilde{\Omega}^{\mp} \subset R_{n}\left(\tilde{x}_{n}\right) \cap$ $R_{n}^{\perp}\left(\tilde{x}_{n}\right) \subset B\left(\tilde{x}_{n} ; \kappa \alpha_{n}\right)$ for $n \in \mathbb{N}$, from which the assertion follows.

6.8. Lemma. Let $\widetilde{S} \in \mathbb{X}$ be a weak solution of Problem 2.1 , and $\left(S_{*, n}^{-}, S_{n}, S_{*, n}^{+}\right)_{n=1}^{\infty}$ an approximating sequence. If $\widetilde{S}$ has the properties stated in Theorem 6.2(a),(b), then for fixed $\alpha \in(0,1)$, we have

$$
\begin{gathered}
\lim _{n \rightarrow \infty} \inf \left\{Q_{n}^{-}(x): x \in S_{n, \alpha}^{-}\right\} \geq \alpha \mathfrak{C}_{0}, \\
\lim _{n \rightarrow \infty} \sup \left\{\left(d\left(x, S_{n}\right) / d\left(x, S_{n}^{+}\right)\right): x \in S_{n, \alpha}^{+}\right\} \leq(\alpha /(1-\alpha)), \\
\lim _{n \rightarrow \infty} \sup \left\{\left(d\left(x, S_{n, \alpha}^{+}\right) / d\left(x, S_{n}^{+}\right)\right): x \in S_{n}\right\} \leq \alpha,
\end{gathered}
$$

where $\mathfrak{C}_{0}>0$ is a constant in Theorem 6.2(a).

Proof. For each $n \in \mathbb{N}$ and $x \in S_{n}$, let $\gamma_{n}^{ \pm}(x)$ be a curve of steepest ascent of $U_{n}^{ \pm}$which joins $x$ to $S_{n}^{ \pm}$. Also define $\gamma_{n, \alpha}^{ \pm}(x)=\gamma_{n}^{ \pm}(x) \cap \Omega_{n, \alpha}^{ \pm}$and $\hat{\gamma}_{n, \alpha}^{ \pm}(x)=$ $\gamma_{n}^{ \pm}(x) \cap \Omega\left(S_{n, \alpha}^{ \pm}, S_{n}^{ \pm}\right)$, and let $\pi_{n, \alpha}^{ \pm}(x)$ denote the point of intersection of $\gamma_{n}^{ \pm}(x)$ with $S_{n, \alpha}^{ \pm}$. Lemma 3.4(f) implies

$$
\begin{gathered}
\pm\left(\alpha \varepsilon_{n}-Q_{n}^{ \pm}\left(\pi_{n, \alpha}^{ \pm}(x)\right)\left|\gamma_{n, \alpha}^{ \pm}(x)\right|\right) \geq 0, \\
\pm\left((1-\alpha) \varepsilon_{n}-Q_{n}^{ \pm}\left(\pi_{n, \alpha}^{ \pm}(x)\right)\left|\hat{\gamma}_{n, \alpha}^{ \pm}(x)\right|\right) \leq 0,
\end{gathered}
$$

both for all $x \in S_{n}$. It follows from (6.8), (6.9) and Lemma 6.6 that

$$
\begin{gathered}
Q_{n}^{-}\left(\pi_{n, \alpha}^{-}(x)\right) \geq\left(\alpha \varepsilon_{n} /\left|\gamma_{n, \alpha}^{-}(x)\right|\right) \geq\left(\alpha \varepsilon_{n} /\left|\gamma_{n}^{-}(x)\right|\right) \\
\geq \alpha \varepsilon_{n} / d\left(x, S_{n}^{-}\right)(1+\zeta(n)) \geq \alpha \mathfrak{C}_{0} /(1+\zeta(n)), \\
\left(d\left(y, S_{n}\right) / d\left(y, S_{n}^{+}\right)\right)(1-\zeta(n)) \leq\left(\left|\gamma_{n, \alpha}^{+}(x)\right| /\left|\hat{\gamma}_{n, \alpha}^{+}(x)\right|\right) \leq(\alpha /(1-\alpha)),
\end{gathered}
$$

for $y=\pi_{n, \alpha}^{+}(x)$, and

$$
\left(d\left(x, S_{n, \alpha}^{+}\right) / d\left(x, S_{n}^{+}\right)\right)(1-\zeta(n)) \leq\left(\left|\gamma_{n, \alpha}^{+}(x)\right| /\left|\gamma_{n}^{+}(x)\right|\right) \leq \alpha,
$$

all uniformly over all $x \in S_{n}$ and $n \in \mathbb{N}$. Since the mappings $\pi_{n, \alpha}^{-}(x): S_{n} \rightarrow S_{n, \alpha}^{-}$, $n \in \mathbb{N}$, are all onto, the assertions follow.

6.9. Lemma. Under Assumptions 2.2(a)-(d), let $\widetilde{S} \in \mathbb{X}$ be a weak solution of Problem 2.1, and let $\left(S_{*, n}^{-}, S_{n}, S_{*, n}^{+}\right)_{n=1}^{\infty}$ be an approximating sequence. Then: (a) For any $\alpha \in\left(0, \alpha_{0}\right)$, we have $Q_{n}^{+}(x) \leq \mathfrak{C}^{+}(\alpha)<\infty$ uniformly over all $x \in S_{n, \alpha}^{+}$ and all sufficiently large $n \in \mathbb{N}$. (b) There exists a null function $z(\alpha):[0,1] \rightarrow \mathbb{R}$ such that $d\left(x, S_{n}\right) \leq \varepsilon_{n} z(\alpha)$ uniformly over all $x \in S_{n, \alpha}^{-}$and all sufficiently large 
$n \in \mathbb{N}$. (c) We have $d\left(x, S_{n, \alpha}^{-}\right) \leq \varepsilon_{n} z(\alpha)(1+\zeta(n))$ uniformly over all $n \in \mathbb{N}$ and $x \in S_{n}$, where $z(\alpha)$ and $\zeta(n)$ are null functions. (d) We have that $d\left(x, S_{n, \alpha}^{-}\right) \leq$ $\mathfrak{C}_{1} z(\alpha)(1+\zeta(n)) d\left(x, S_{n}^{-}\right)$, uniformly for all $x \in S_{n}$ and $n \in \mathbb{N}$.

Proof. Parts (a) and (b) follow from Lemma 4.10 (eq. (4.3)) and Lemma 4.11 (eq. $(4.5)$ ), respectively (where we define $z(\alpha)=z_{2}^{-1}(\alpha)$ ), and Part (d) follows from Part (c) and Lemma 4.8. It remains to prove Part (c). Let $\alpha \in(0,1)$ be fixed. For any $n \in \mathbb{N}$ and $x \in S_{n}$, choose $y \in S_{n, \alpha}^{-}$and $z \in S_{n}$ such that $|y-x|=d\left(x, S_{n, \alpha}^{-}\right)$ and $|z-y|=d\left(y, S_{n}\right)$. Clearly $(x-y)=|x-y| \nu_{n}(y),(z-y)=|z-y| \nu_{n}(z)$, and

$$
d\left(x, S_{n, \alpha}^{-}\right)=|x-y| \leq\left(|y-z| /\left[\nu_{n}(y) \cdot \nu_{n}(z)\right]\right) \leq \varepsilon_{n} z(\alpha) /\left[\nu_{n}(y) \cdot \nu_{n}(z)\right],
$$

where the above inequalities are due to Part (b) and the convexity of $S_{n}$. However, it follows from Lemmas 6.4 and 6.5 that $\left|\nu_{n}(y)-\nu_{n}(z)\right| \leq \zeta(n)$, uniformly over all $n \in \mathbb{N}, y \in S_{n, \alpha}^{-}$and $z \in S_{n}$ such that $|z-y|=d\left(y, S_{n}\right) \leq \varepsilon_{n} z(\alpha)$.

6.10. Lemma. Under Assumptions $2.2(\mathrm{a})-(\mathrm{d})$, let $\widetilde{S} \in \mathbb{X}$ be a weak solution of Problem 2.1, and let $\left(S_{*, n}^{-}, S_{n}, S_{*, n}^{+}\right)_{n=1}^{\infty}$ be an approximating sequence (corresponding to a positive null sequence $\left.\left(\varepsilon_{n}\right)_{n=1}^{\infty}\right)$. Then for any point $x_{0} \in \widetilde{S}$, there exist sequences $\left(x_{n}^{ \pm}\right)_{n=1}^{\infty}$ such that $x_{n}^{ \pm} \rightarrow x_{0}$ as $n \rightarrow \infty, x_{n}^{ \pm} \in \Omega_{n}^{ \pm}$for all $n \in \mathbb{N}$, and

$$
\limsup _{n \rightarrow \infty} F\left(x_{0}, Q_{n}^{-}\left(x_{n}^{-}\right), Q_{n}^{+}\left(x_{n}^{+}\right)\right) \leq 0 \text {. }
$$

Proof. Choose sequences $\left(x_{n}\right)_{n=1}^{\infty}$ and $\left(y_{n}^{ \pm}\right)_{n=1}^{\infty}$ such that $x_{n} \in S_{n}$ and $y_{n}^{ \pm} \in \Omega_{n}^{ \pm}$for each $n \in \mathbb{N}$, and such that $x_{n} \rightarrow x_{0},\left(\left|y_{n}^{ \pm}-x_{n}\right| / \varepsilon_{n}\right) \rightarrow 0$, and $\left(U_{n}^{ \pm}\left(y_{n}^{ \pm}\right) / \varepsilon_{n}\right) \rightarrow 0$, all as $n \rightarrow \infty$. For each $n \in \mathbb{N}$, we have $F\left(x_{n},\left[\varepsilon_{n} / d\left(x_{n}, S_{n}^{-}\right)\right],\left[\varepsilon_{n} / d\left(x_{n}, S_{n}^{+}\right)\right]\right)=0$. Since $\mathfrak{C}_{0} \leq\left[\varepsilon_{n} / d\left(x_{n}, S_{n}^{ \pm}\right)\right] \leq \mathfrak{C}_{1}$ uniformly for all sufficiently large $n \in \mathbb{N}$ (due to Lemma 4.8), we conclude from the continuity of $F$ that for any $\eta>0$, we have

$$
F\left(x_{0},\left[\varepsilon_{n} / d\left(x_{n}, S_{n}^{-}\right)\right],\left[\varepsilon_{n} / d\left(x_{n}, S_{n}^{+}\right)\right]\right)<\eta
$$

for all sufficiently large $n \in \mathbb{N}$. Let $\gamma_{n}^{ \pm}$denote the (unique) arc of steepest ascent of $U_{n}^{ \pm}$joining $y_{n}^{ \pm}$to the point $z_{n}^{ \pm} \in S_{n}^{ \pm}$. Then it follows from Lemmas 3.4(f) and 6.6 that

$$
\begin{gathered}
Q_{n}^{+}\left(z_{n}^{+}\right) \leq\left(\varepsilon_{n} /\left|\gamma_{n}^{+}\right|\right) \leq\left(\varepsilon_{n} / d\left(y_{n}^{+}, S_{n}^{+}\right)\right) \\
\leq\left[\varepsilon_{n} / d\left(x_{n}, S_{n}^{+}\right)\right]\left(1-\left(\left|y_{n}^{+}-x_{n}\right| / d\left(x_{n}, S_{n}^{+}\right)\right)\right)^{-1} \leq\left[\varepsilon_{n} / d\left(x_{n}, S_{n}^{+}\right)\right](1+\zeta(n)), \\
Q_{n}^{-}\left(z_{n}^{-}\right) \geq\left(\left(\varepsilon_{n}-U_{n}^{-}\left(y_{n}^{-}\right)\right) /\left|\gamma_{n}^{-}\right|\right) \geq\left[\varepsilon_{n} / d\left(y_{n}^{-}, S_{n}^{-}\right)\right](1-\zeta(n)) \\
\geq\left[\varepsilon_{n} / d\left(x_{n}, S_{n}^{-}\right)\right](1-\zeta(n))\left(1+\left(\left|y_{n}^{-}-x_{n}\right| / d\left(x_{n}, S_{n}^{-}\right)\right)\right)^{-1} \\
\geq\left[\varepsilon_{n} / d\left(x_{n}, S_{n}^{-}\right)\right](1-\zeta(n)) .
\end{gathered}
$$

In view of $(6.10),(6.11),(6.12)$, it follows from Assumption 2.2(a) that for any given $\eta>0$, we have $F\left(x_{0}, Q_{n}^{-}\left(z_{n}^{-}\right), Q_{n}^{+}\left(z_{n}^{+}\right)\right)<\eta$ for $n \in \mathbb{N}$ sufficiently large. Finally, for each sufficiently large $n \in \mathbb{N}$, one applies the continuity of the function $F\left(x_{0}, p, q\right)$ and the continuity and positivity of the functions $Q_{n}^{ \pm}(x)$ to choose the points $x_{n}^{ \pm} \in$ $\Omega_{n}^{ \pm}$so close to the corresponding points $z_{n}^{ \pm} \in S_{n}^{ \pm}$that $F\left(x_{0}, Q_{n}^{-}\left(x_{n}^{-}\right), Q_{n}^{+}\left(x_{n}^{+}\right)\right)<\eta$.

6.11. Lemma. Under Assumptions 2.2(a)-(d), let $\widetilde{S} \in \mathbb{X}$ be a weak solution of Problem 2.1, and let $\left(S_{*, n}^{-}, S_{n}, S_{*, n}^{+}\right)_{n=1}^{\infty}$ be an approximating sequence. For fixed $x_{0} \in \widetilde{S}$, choose Cartesian coordinates such that $x_{0}$ lies at the origin and $\tilde{\nu}_{0}=$ $\tilde{\nu}\left(x_{0}\right)=e_{N}:=(0, \ldots, 0,1)$. In the new coordinates, let $E_{\delta}=\mathfrak{D}_{\delta} \times[-\delta, \delta]$ for 
$\delta>0$, where $\mathfrak{D}_{\delta}=\left\{y \in \mathbb{R}^{N-1}:|y| \leq \delta\right\}$. Then: (a) for sufficiently small $\delta>0$ and sufficiently large $n$ (and for any $\alpha \in(0,1)$ ), the intersections of the surfaces $\widetilde{S}, S_{n}, S_{n}^{ \pm}, S_{n, \alpha}^{ \pm}$with $E_{\delta}$ are respectively the graphs of Lipschitz-continuous functions $z=\widetilde{S}(y), S_{n}(y), S_{n}^{ \pm}(y), S_{n, \alpha}^{ \pm}(y): \mathfrak{D}_{\delta} \rightarrow \mathbb{R}$. Moreover, the Lipschitz constants of these functions are of order $z(\delta)$ (in the case of $\left.\widetilde{S}(y): \mathfrak{D}_{\delta} \rightarrow \mathbb{R}\right)$ and $z(\delta, n)$ (in the case of $\left.S_{n}(y), S_{n}^{ \pm}(y), S_{n, \alpha}^{ \pm}(y): \mathfrak{D}_{\delta} \rightarrow \mathbb{R}\right)$ as $\delta \downarrow 0$ and $n \rightarrow \infty$. (b) For sufficiently small $\delta>0$, we have that

$$
S_{n}(y), S_{n}^{ \pm}(y), S_{n, \alpha}^{ \pm}(y) \rightarrow \widetilde{S}(y) \text { as } n \rightarrow \infty
$$

all uniformly in $\mathfrak{D}_{\delta}$. (c) We have that

$$
\left|S_{n, \alpha}^{ \pm}(y)-S_{n}(y)\right| \leq\left(1+z\left(\delta, n_{0}\right)\right) z^{ \pm}(\alpha) d\left(x_{n}(y), S_{n}^{ \pm}\right),
$$

uniformly over all $\alpha \in(0,1), y \in \mathfrak{D}_{\delta}$, and $n \geq n_{0}$, where $x_{n}(y)=\left(y, S_{n}(y)\right)$ and where $z^{ \pm}(\alpha):(0,1) \rightarrow \mathbb{R}_{+}$denote fixed functions such that $z^{ \pm}(\alpha) \downarrow 0$ as $\alpha \downarrow 0$.

Proof. It follows from Lemma 4.8 and the definition of a weak solution that $d\left(x_{0}, S_{n}\right), d\left(x_{0}, S_{n}^{ \pm}\right), d\left(x_{0}, S_{n, \alpha}^{ \pm}\right) \rightarrow 0$, all as $n \rightarrow \infty$. By Lemmas 6.4 and 6.5 , we have $\left|\tilde{\nu}(x)-\tilde{\nu}_{0}\right| \leq z(\delta)$ and $\left|\nu_{n}(x)-\tilde{\nu}_{0}\right| \leq z(\delta, n)$ uniformly in $B_{\delta}\left(x_{0}\right) \backslash S_{n}$ as $\delta \downarrow 0$ and $n \rightarrow \infty$. Part (a) easily follows from this. Part (b) also follows from Lemmas 4.8, 6.4, and 6.5. Turning to the proof of Part (c), let $y \in \mathfrak{D}_{\delta}$ correspond to the point $x_{n}=\left(y, z_{n}\right)=\left(y, S_{n}(y)\right) \in S_{n}$. Then $d\left(x_{n}, S_{n, \alpha}^{ \pm}\right) \rightarrow 0$ as $n \rightarrow \infty$, by Lemma 4.8 . For $n \in \mathbb{N}$ sufficiently large (depending on $\delta>0$ ), we have $d\left(x_{n}, S_{n, \alpha}^{ \pm}\right)=\left|x_{n}-x_{n, \alpha}^{ \pm}\right|$, where $x_{n, \alpha}^{ \pm}=\left(y_{n, \alpha}^{ \pm}, z_{n, \alpha}^{ \pm}\right) \in E_{2 \delta} \cap S_{n, \alpha}^{ \pm}$. Clearly $\left|z_{n, \alpha}^{ \pm}-z_{n}\right| \leq d\left(x_{n}, S_{n, \alpha}^{ \pm}\right)$. Also, the vector $\left(x_{n, \alpha}^{ \pm}-x_{n}\right)$ is parallel to $\pm \nu_{n}\left(x_{n, \alpha}^{ \pm}\right)$(where $\left|\nu_{n}\left(x_{n, \alpha}^{ \pm}\right)-\tilde{\nu}_{0}\right| \leq z(\delta, n)$ by Lemmas 6.4 and 6.5). Therefore $\left|y-y_{n, \alpha}^{ \pm}\right| \leq d\left(x_{n}, S_{n, \alpha}^{ \pm}\right) z(\delta, n)$, whence

$$
\left|z_{n, \alpha}^{ \pm}-S_{n, \alpha}^{ \pm}(y)\right| \leq L(\delta, n)\left|y-y_{n, \alpha}^{ \pm}\right| \leq L(\delta, n) d\left(x_{n}, S_{n, \alpha}^{ \pm}\right) z(\delta, n),
$$

where $L(\delta, n)$ denotes a uniform Lipschitz constant for the functions $S_{n, \alpha}^{ \pm}: \mathfrak{D}_{\delta} \rightarrow \mathbb{R}$, $\alpha \in(0,1)$. By combining the above inequalities, one sees that

$\left|S_{n, \alpha}^{ \pm}(y)-S_{n}(y)\right| \leq\left|S_{n, \alpha}^{ \pm}(y)-z_{n, \alpha}^{ \pm}\right|+\left|z_{n, \alpha}^{ \pm}-z_{n}\right| \leq d\left(x_{n}, S_{n, \alpha}^{ \pm}\right)(1+L(\delta, n)) z(\delta, n)$,

where the estimate is easily seen to hold uniformly over all $y \in \mathfrak{D}_{\delta}$. Finally, we have $d\left(x, S_{n, \alpha}^{ \pm}\right) \leq z^{ \pm}(\alpha)(1+\zeta(n)) d\left(x, S_{n}^{ \pm}\right)$uniformly for all $x \in S_{n}$, as follows from Lemma 6.8 (eq. (6.7)) and Lemma 6.9(d). The assertion follows.

6.12. Lemma. In the context of Lemma 6.11, for fixed $x_{0} \in \widetilde{S}$ and fixed, sufficiently small $\delta>0$, let $z=\widetilde{S}(y), S_{n}(y), S_{n}^{ \pm}(y), S_{n, \alpha}^{ \pm}(y): \mathfrak{D}_{\delta} \rightarrow \mathbb{R}$ be the representations of the intersections with $E_{\delta}$ of the surfaces $\widetilde{S}, S_{n}, S_{n}^{ \pm}, S_{n, \alpha}^{ \pm}$. Then for any $\eta>0$, there exists a value $\alpha_{0}=\alpha_{0}(\eta) \in(0,1)$ such that for any fixed $\alpha \in\left(0, \alpha_{0}\right]$, and for sufficiently large $n \in \mathbb{N}$ (depending on $\alpha$ ), we have

$$
F\left(\tilde{x}(y), Q_{n}^{-}\left(x_{n, \alpha}^{-}(y)\right), Q_{n}^{+}\left(x_{n, \alpha}^{+}(y)\right)\right)>-\eta
$$

uniformly over all $y \in \mathfrak{D}_{\delta}$, where $\tilde{x}(y)=(y, \widetilde{S}(y)), x_{n}(y)=\left(y, S_{n}(y)\right)$, and $x_{n, \alpha}^{ \pm}(y)$ $=\left(y, S_{n, \alpha}^{ \pm}(y)\right)$. (b) For any small $\alpha \in(0,1)$, there exist positive values $\mathfrak{C}^{ \pm}(\alpha)$ such that $Q_{n}^{+}(x) \leq \mathfrak{C}^{+}(\alpha)$ on $S_{n, \alpha}^{+}$and $Q_{n}^{-}(x) \geq \mathfrak{C}^{-}(\alpha)$ on $S_{n, \alpha}^{-}$, both uniformly for all sufficiently large $n \in \mathbb{N}$.

Proof. We observe that $F\left(x,\left[\varepsilon_{n} / d\left(x, S_{n}^{-}\right)\right],\left[\varepsilon_{n} / d\left(x, S_{n}^{+}\right)\right]\right)=0$ for all $x \in S_{n}$ and $n \in \mathbb{N}$. In view of this, it follows from the continuity of the function $F(x, p, q)$ (and 
the fact that $\mathfrak{C}_{0} \leq\left[\varepsilon_{n} / d\left(x, S_{n}^{ \pm}\right)\right] \leq \mathfrak{C}_{1}$ for all $n \in \mathbb{N}$ and $\left.x \in S_{n}\right)$ that if $n \in \mathbb{N}$ is sufficiently large, then

$$
F\left(\tilde{x}(y),\left[\varepsilon_{n} / d\left(x_{n}(y), S_{n}^{-}\right)\right],\left[\varepsilon_{n} / d\left(x_{n}(y), S_{n}^{+}\right)\right]\right)>-(\eta / 2)
$$

for all $y \in \mathfrak{D}_{\delta}$. For sufficiently large $n \in \mathbb{N}$, let $\gamma:=\gamma_{n, \alpha}^{ \pm}(y)$ denote the arc of steepest ascent of $U_{n}^{ \pm}$joining the point $x_{n, \alpha}^{ \pm}(y):=\left(y, S_{n, \alpha}^{ \pm}(y)\right)$ to the surface $S_{n}^{ \pm}$. Then $\left|\gamma_{n, \alpha}^{+}(y)\right| \leq d\left(x_{n, \alpha}^{+}(y), S_{n}^{+}\right)(1+\zeta(n))$ for all $n \in \mathbb{N}$ (where $\zeta(n) \rightarrow 0$ as $\left.n \rightarrow \infty\right)$, by Lemma 6.6 (and obviously $\left|\gamma_{n, \alpha}^{ \pm}(y)\right| \geq d\left(x_{n, \alpha}^{ \pm}(y), S_{n}^{ \pm}\right)$). Therefore

$$
\begin{aligned}
(1-\alpha) \varepsilon_{n} & =\int_{\gamma} Q_{n}^{+}(x)|d x| \leq Q_{n}^{+}\left(x_{n, \alpha}^{+}(y)\right)\left|\gamma_{n, \alpha}^{+}(y)\right| \\
& \leq(1+\zeta(n)) Q_{n}^{+}\left(x_{n, \alpha}^{+}(y)\right) d\left(x_{n, \alpha}^{+}(y), S_{n}^{+}\right) \\
& \leq(1+\zeta(n)) Q_{n}^{+}\left(x_{n, \alpha}^{+}(y)\right)\left[d\left(x_{n}(y), S_{n}^{+}\right)+\left|S_{n, \alpha}^{+}(y)-S_{n}(y)\right|\right] \\
& \leq(1+\zeta(n)) Q_{n}^{+}\left(x_{n, \alpha}^{+}(y)\right) d\left(x_{n}(y), S_{n}^{+}\right)\left[1+(1+z(\delta, n)) z^{+}(\alpha)\right]
\end{aligned}
$$

for sufficiently large $n \in \mathbb{N}$ (by Lemma 6.11(c)), from which it follows that

$$
Q_{n}^{+}\left(x_{n, \alpha}^{+}(y)\right) \geq\left((1-\alpha) /(1+\zeta(n))\left[(1+z(\delta, n)) z^{+}(\alpha)\right]\right)\left[\varepsilon_{n} / d\left(x_{n}(y), S_{n}^{+}\right)\right]
$$

for sufficiently large $n \in \mathbb{N}$. A similar argument using Lemma 6.11(c) shows that

$$
\begin{aligned}
(1-\alpha) \varepsilon_{n} & =\int_{\gamma} Q_{n}^{-}(x)|d x| \geq Q_{n}^{-}\left(x_{n, \alpha}^{-}(y)\right)\left|\gamma_{n, \alpha}^{-}(y)\right| \geq Q_{n}^{-}\left(x_{n, \alpha}^{-}(y)\right) d\left(x_{n, \alpha}^{-}(y), S_{n}^{-}\right) \\
& \geq Q_{n}^{-}\left(x_{n, \alpha}^{-}(y)\right)\left(d\left(x_{n}(y), S_{n}^{-}\right)-\left|S_{n, \alpha}^{-}(y)-S_{n}(y)\right|\right) \\
& \geq Q_{n}^{-}\left(x_{n, \alpha}^{-}(y)\right) d\left(x_{n}(y), S_{n}^{-}\right)\left[1-(1+z(\delta, n)) z^{-}(\alpha)\right],
\end{aligned}
$$

from which it follows that

$$
Q_{n}^{-}\left(x_{n, \alpha}^{-}(y)\right) \leq\left((1-\alpha) /\left[1-(1+z(\delta, n)) z^{-}(\alpha)\right]\right)\left[\varepsilon_{n} / d\left(x_{n}(y), S_{n}^{-}\right)\right]
$$

for sufficiently large $n \in \mathbb{N}$, and for all $y \in \mathfrak{D}_{\delta}$ and $\alpha \in(0,1)$. Now Part (a) follows from eqs. (6.13), (6.14), and (6.15), and the assumed continuity and monotonicity properties of the function $F(x, p, q)$. Finally, regarding Part (b), for $\alpha \in(0,1)$ sufficiently small and $n \in \mathbb{N}$ sufficiently large, we have $Q_{n}^{+}(x) \leq \mathfrak{C}^{+}(\alpha)$ for all $x \in S_{n, \alpha}^{+}$, due to Lemma $6.9(\mathrm{a})$, while $Q_{n}^{-}(x) \geq \mathfrak{C}^{-}(\alpha):=\left(\alpha \mathfrak{C}_{0} / 2\right)$ for all $x \in S_{n, \alpha}^{-}$, due to Lemma 6.8, eq. (6.5).

\section{Proof that $F\left(x_{0}, \widetilde{Q}^{-}\left(x_{0}\right), \widetilde{Q}^{+}\left(x_{0}\right)\right) \leq 0$ For all $x_{0} \in \widetilde{S}$}

7.1. Theorem. Under Assumptions $2.2(\mathrm{a})-(\mathrm{d})$, let $\widetilde{S} \in \mathbb{X}$ be a weak solution of Problem 2.1. Then, in the notation of $\S 6.1$, we have $F\left(x_{0}, \widetilde{Q}^{-}\left(x_{0}\right), \widetilde{Q}^{+}\left(x_{0}\right)\right) \leq 0$ for all $x_{0} \in \widetilde{S}$.

7.2. Proposition. Let $\widetilde{S}$ be a weak solution of Problem 2.1 (with the properties stated in Theorem 6.2(a),(b)), and let $\left(S_{*, n}^{-}, S_{n}, S_{*, n}^{+}\right)_{n=1}^{\infty}$ be a corresponding approximating sequence. Let the sequences $\left(\tilde{x}_{n}\right)$ and $\left(x_{n}^{ \pm}\right)$be given such that $\tilde{x}_{n} \in \widetilde{S}, x_{n}^{ \pm} \in \widetilde{\Omega}^{ \pm} \cap \Omega_{*, n}^{ \pm}$and $x_{n}^{ \pm}-\tilde{x}_{n} \rightarrow 0$ as $n \rightarrow \infty$. For $n \in \mathbb{N}$, let $\tilde{\gamma}_{n}^{ \pm}$denote the curve of steepest ascent of $\widetilde{U}^{ \pm}$initiating at $\tilde{x}_{n}$, and let $\tilde{p}_{n}^{ \pm}(t):[0,1) \rightarrow \tilde{\gamma}_{n}^{ \pm}$denote the parametrization of $\tilde{\gamma}_{n}^{ \pm}$such that $\widetilde{U}^{ \pm}\left(\tilde{p}_{n}^{ \pm}(t)\right)=t$ for all $t \in[0,1)$. Also for $n \in \mathbb{N}$, let $\gamma_{n}^{ \pm}$denote the maximal curve of steepest ascent of $U_{n}^{ \pm}$passing through the point $x_{n}^{ \pm}$, and, for sufficiently small $b_{0} \in(0,1)$, let $p_{n}^{ \pm}(t):\left(\alpha_{n}^{ \pm}, b_{0}\right) \rightarrow \gamma_{n}^{ \pm}$denote the 
parametrization of $\gamma_{n}^{ \pm} \cap\left\{\widetilde{U}^{ \pm}<b_{0}\right\}$ such that $\widetilde{U}^{ \pm}\left(p_{n}^{ \pm}(t)\right)=t$ for $t \in\left(\alpha_{n}^{ \pm}, b_{0}\right)$. Then $p_{n}^{ \pm}(t)-\tilde{p}_{n}^{ \pm}(t) \rightarrow 0$ as $n \rightarrow \infty$ for $t \in\left(0, b_{0}\right)$.

Proof. For simplicity, we eliminate " \pm " from the notation, so that $S_{*}^{ \pm}, \widetilde{\Omega}^{ \pm}, \widetilde{U}^{ \pm}(x)$, $\widetilde{Q}^{ \pm}(x), \tilde{p}_{n}^{ \pm}(t), S_{*, n}^{ \pm}, \Omega_{*, n}^{ \pm}, U_{n}^{ \pm}(x), Q_{n}^{ \pm}(x)$, and $p_{n}^{ \pm}(t)$, become $S_{*}, \widetilde{\Omega}, \widetilde{U}(x), \widetilde{Q}(x), \tilde{p}_{n}(t)$, $S_{*, n}, \Omega_{*, n}, U_{n}(x), Q_{n}(x)$, and $p_{n}(t)$. Choose $b_{0} \in(0,1)$ and $n_{0} \in \mathbb{N}$ such that $2 d(x, \widetilde{S}) \leq d\left(x, S_{*, n}\right)$ and $\mathfrak{C}_{0} \leq \widetilde{Q}(x) \leq \mathfrak{C}_{1}$, both for all $x \in\left\{\widetilde{U}(x) \leq b_{0}\right\}$, and such that $\nabla \widetilde{U}(x) \cdot \nabla U_{n}(x) \geq(1 / 2) \widetilde{\widetilde{Q}}(x) Q_{n}(x)$ throughout $\left\{\widetilde{U}(x) \leq b_{0}\right\} \cap \Omega_{*, n}$ for $n \geq n_{0}$. (This choice of $b_{0}$ and $n_{0}$ is possible due to Theorem 6.2(a) and Lemma 6.5). There exist a uniform constant $A_{0}>0$ and a null function $\zeta_{0}(n)$ such that

$$
d\left(\tilde{p}_{n}(t), \partial\left(\widetilde{\Omega} \cap \Omega_{*, n}\right)\right) \geq A_{0} t-\zeta_{0}(n) ; d\left(p_{n}(t), \partial\left(\widetilde{\Omega} \cap \Omega_{*, n}\right)\right) \geq A_{0} t-\zeta_{0}(n)
$$

for $t \in\left(\alpha_{n}, b_{0}\right)$, independent of $n \in \mathbb{N}$. This follows from the fact that $\Delta\left(\widetilde{S}, S_{n}\right) \rightarrow 0$ as $n \rightarrow \infty$ and $d(x, \widetilde{S}) \geq A_{0} t$ for all $x \in \widetilde{\Omega}$ such that $\widetilde{U}(x)=t$ (by Theorem $6.2(\mathrm{a})$ ). Clearly, we have

$$
\tilde{p}_{n}^{\prime}(t)=\widetilde{V}\left(\tilde{p}_{n}(t)\right) \text { for } t \in[0,1),
$$

where $\widetilde{V}(x)=\nabla \widetilde{U}(x) / \widetilde{Q}^{2}(x)$ in $\widetilde{\Omega} \cup \widetilde{S}$. Also,

$$
p_{n}^{\prime}(t)=V_{n}\left(p_{n}(t)\right) \quad \text { for } t \in\left(\alpha_{n}, b_{0}\right),
$$

where $V_{n}(x)=\nabla U_{n}(x) /\left(\nabla U_{n}(x) \cdot \nabla \widetilde{U}(x)\right)$ in $\left\{\widetilde{U}(x)<b_{0}\right\} \cap \Omega_{*, n}$. Let $\delta_{n}(t)=$ $\left|p_{n}(t)-\tilde{p}_{n}(t)\right|$ for $t \in\left(\alpha_{n}, b_{0}\right)$. Then

$$
\delta_{n}(t) \leq\left|x_{n}-\tilde{x}_{n}\right|+\left|\tilde{p}_{n}(t)-\tilde{p}_{n}(0)\right|+\left|p_{n}(t)-p_{n}\left(t_{n}\right)\right|,
$$

where we choose $t_{n} \in\left(\alpha_{n}, b_{0}\right)$ such that $p_{n}\left(t_{n}\right)=x_{n}$. However, we have

$$
\left|\tilde{p}_{n}(t)-\tilde{p}_{n}(0)\right| \leq \int_{0}^{t}\left|\widetilde{V}\left(\tilde{p}_{n}\left(t^{\prime}\right)\right)\right| d t^{\prime} \leq\left(t / \mathfrak{C}_{0}\right)
$$

for $t \in\left(0, b_{0}\right)$, since $|\widetilde{V}(x)|=(1 / \widetilde{Q}(x)) \leq\left(1 / \mathfrak{C}_{0}\right)$ in $\left\{\widetilde{U}(x)<b_{0}\right\}$. Similarly,

$$
\left|p_{n}(t)-p_{n}\left(t_{n}\right)\right| \leq \int_{t_{n}}^{t}\left|V_{n}\left(p_{n}\left(t^{\prime}\right)\right)\right| d t^{\prime} \leq\left(2 / \mathfrak{C}_{0}\right)\left(t-t_{n}\right)
$$

for $n \geq n_{0}$ and $t \in\left(t_{n}, b_{0}\right)$, since $\left|V_{n}(x)\right| \leq(2 / \widetilde{Q}(x)) \leq\left(2 / \mathfrak{C}_{0}\right)$ in $\left\{\widetilde{U}(x)<b_{0}\right\} \cap \Omega_{*, n}$ for $n \geq n_{0}$. Also, $\left|x_{n}-\tilde{x}_{n}\right| \rightarrow 0$ and $t_{n}=\widetilde{U}\left(x_{n}\right) \rightarrow 0$ as $n \rightarrow \infty$. Therefore, it follows from (7.2), (7.3), and (7.4) that there exist a uniform constant $C$ and a null function $\zeta_{1}(n)$ such that

$$
\delta_{n}(t) \leq C t+\zeta_{1}(n)
$$

uniformly for all $t \in\left(\alpha_{n}, b_{0}\right)$ and all $n \in \mathbb{N}$. Also, we have that

$$
\delta_{n}^{\prime}(t) \leq\left|V_{n}\left(p_{n}(t)\right)-\widetilde{V}\left(\tilde{p}_{n}(t)\right)\right| \leq\left|V_{n}\left(p_{n}(t)\right)-\widetilde{V}\left(p_{n}(t)\right)\right|+\left|\widetilde{V}\left(p_{n}(t)\right)-\widetilde{V}\left(\tilde{p}_{n}(t)\right)\right| .
$$

Now $\max \left\{\left|U_{n}(x)-\widetilde{U}(x)\right|: x \in \operatorname{Cl}\left(\widetilde{\Omega} \cap \Omega_{*, n}\right)\right\} \leq \zeta_{2}(n)$ as $n \rightarrow \infty$, from which it follows from a standard estimate for derivatives of harmonic functions (see [18], $\S 2.7)$ that

$$
q_{n}(x):=\left|\nabla\left(U_{n}(x)-\widetilde{U}(x)\right)\right| \leq R_{n}(x):=N \zeta_{2}(n) / d\left(x, \partial\left(\widetilde{\Omega} \cap \Omega_{*, n}\right)\right)
$$


relative to the region $\left\{\widetilde{U}(x)<b_{0}\right\}$. However, a direct calculation based on the definitions of $\widetilde{V}(x)$ and $V_{n}(x)$ shows that

$$
\left|V_{n}(x)-\widetilde{V}(x)\right| \leq 2 q_{n}(x) /\left|\nabla U_{n}(x) \cdot \nabla \widetilde{U}(x)\right| \leq 4 q_{n}(x) /\left(\widetilde{Q}(x)\left[\widetilde{Q}(x)-q_{n}(x)\right]\right)
$$

for any $x \in\left\{\widetilde{U}(x)<b_{0}\right\} \cap \Omega_{*, n}$ such that $q_{n}(x)<\widetilde{Q}(x)$. Since $\widetilde{Q}(x) \geq \mathfrak{C}_{0}$ in $\left\{\widetilde{U}(x)<b_{0}\right\}$, it follows from (7.7) and (7.8) that

$$
\left|V_{n}(x)-\tilde{V}(x)\right| \leq 4 R_{n}(x) /\left[\mathfrak{C}_{0}\left(\mathfrak{C}_{0}-R_{n}(x)\right)\right]
$$

whenever $R_{n}(x)<\mathfrak{C}_{0}$ in $\left\{\widetilde{U}(x)<b_{0}\right\} \cap \Omega_{n}$. It follows by substituting (7.1) into (7.9) that

$$
\left|V_{n}\left(p_{n}(t)\right)-\widetilde{V}\left(p_{n}(t)\right)\right| \leq \zeta_{3}(n) /\left(t-\zeta_{3}(n)\right),
$$

for $t \in\left(\zeta_{3}(n), b_{0}\right)$, where $\zeta_{3}(n)$ denotes a (specific) null function. Let $L_{n}(t)$ denote the straight line-segment joining $\tilde{p}_{n}(t)$ to $p_{n}(t)$, and let $\gamma_{n}(t)$ denote the projection of $L_{n}(t)$ on the surface $\{\widetilde{U}=t\}$ along radial lines emanating from the origin (located inside $\left.D_{*}^{-}\right)$. Clearly $\gamma_{n}(t)$ is a smooth arc in $\{\widetilde{U}=t\}$ which joins $\tilde{p}_{n}(t)$ to $p_{n}(t)$. Moreover, $\left|\gamma_{n}(t)\right| \leq M_{0}\left|L_{n}(t)\right|=M_{0} \delta_{n}(t)$ for some uniform constant $M_{0}$, where $\left|\gamma_{n}(t)\right|$ denotes the length of the $\operatorname{arc} \gamma_{n}(t)$. We have that

$$
\widetilde{Q}^{2}\left(x^{*}\right)\left(\partial \widetilde{V}^{*}\left(x^{*}\right) / \partial \tau\right)=(\nabla-2 \partial / \partial \tilde{\nu})\left(\partial \widetilde{U}\left(x^{*}\right) / \partial \tau\right)
$$

at any (fixed) point $x^{*} \in\{\widetilde{U}=t\}$, where $\tilde{\nu}=\tilde{\nu}\left(x^{*}\right):=\nabla \widetilde{U}\left(x^{*}\right) / \widetilde{Q}\left(x^{*}\right)$ and $\tau \perp \tilde{\nu}$. By Lemma 6.4, we have $|\tilde{\nu}(x)-\tilde{\nu}(y)| \leq z(t)$ for all $x, y \in \widetilde{\Omega}$ such that $|x-y| \leq$ $2 t$. Since $\partial \widetilde{U}\left(x^{*}\right) / \partial \tau=0$, it follows that $|\partial \widetilde{U}(x) / \partial \tau| \leq z(t)$ for all $x \in \widetilde{\Omega}$ such that $\left|x-x^{*}\right| \leq 2 t$. It follows by (7.1) and a standard derivative estimate that $\left|\partial \widetilde{V}\left(x^{*}\right) / \partial \tau\right| \leq z(t) / t$. Since this estimate holds uniformly for all points $x^{*} \in \gamma_{n}(t)$, it follows that

$$
\left|\widetilde{V}\left(p_{n}(t)\right)-\widetilde{V}\left(\tilde{p}_{n}(t)\right)\right| \leq(z(t) / t) \delta_{n}(t) .
$$

It follows by combining (7.6), (7.10), and (7.12) that

$$
\delta_{n}^{\prime}(t) \leq(z(t) / t) \delta_{n}(t)+2 \zeta_{3}(n) / t
$$

for all $n \geq n_{0}$ and $t \in\left(\alpha_{n}, b_{0}\right)$ such that $t \geq 2 \zeta_{3}(n)$. Choose $b_{1} \in\left(0, b_{0}\right)$ sufficiently small, so that $z\left(b_{1}\right)<1 / 2$. Let $\left(a_{n}\right)$ denote a positive null sequence such that $a_{n}>\max \left\{\alpha_{n}, \zeta_{3}(n)\right\}$ for each $n \in \mathbb{N}$. For any value $b \in\left(0, b_{1}\right)$, and for sufficiently large $n \in \mathbb{N}$ (so that $a_{n}<b$ ), it follows by integrating (7.13) on the interval $\left[a_{n}, b\right]$ that

$$
\delta_{n}(b) \leq \delta_{n}\left(a_{n}\right)\left(b / a_{n}\right)^{z(b)}+\left(\zeta_{3}(n) / z(b)\right)\left(\left(b / a_{n}\right)^{z(b)}-1\right) .
$$

But $\delta_{n}\left(a_{n}\right) \leq C a_{n}+\zeta_{1}(n)$, due to (7.5). By substituting this inequality into (7.14), one concludes that

$$
\delta_{n}(b) \leq\left(b^{z(b)} / z(b)\right)\left(C z(b) a_{n}^{1-z(b)}+\left[\zeta_{3}(n)+z(b) \zeta_{1}(n)\right] / a_{n}^{z(b)}\right) .
$$

However, one can easily choose the null sequence $\left(a_{n}\right)$ such that

$$
\left(\left[\zeta_{3}(n)+z(b) \zeta_{1}(n)\right] / a_{n}^{z(b)}\right) \rightarrow 0
$$

as $n \rightarrow \infty$. Therefore, we conclude that $\delta_{n}(b) \rightarrow 0$ as $n \rightarrow \infty$, for any fixed value $b \in\left(0, b_{1}\right)$. 
7.3. Lemma. Let $\widetilde{S}$ be a weak solution of Problem 2.1 (having the properties stated in Theorem 6.2(a), (b)), and let $\left(S_{*, n}^{-}, S_{n}, S_{*, n}^{+}\right)_{n=1}^{\infty}$ be a corresponding approximating sequence. Given the sequences $\left(x_{n}^{ \pm}\right)$and $\left(\tilde{x}_{n}^{ \pm}\right)$such that $x_{n}^{ \pm}, \tilde{x}_{n}^{ \pm} \rightarrow x_{0}^{ \pm} \in \widetilde{\Omega}^{ \pm}$, let $\left(\tilde{\gamma}_{n}^{ \pm}\right)$denote the sequence of arcs of steepest descent of $\widetilde{U}^{ \pm}$joining $\tilde{x}_{n}^{ \pm}$to $\widetilde{S}$, and let $\left(\gamma_{n}^{ \pm}\right)$denote the sequence of arcs of steepest descent of $U_{n}^{ \pm}$, joining $x_{n}^{ \pm}$to $S_{n}$. Then $\Delta\left(\tilde{\gamma}_{n}^{ \pm}, \gamma_{n}^{ \pm}\right) \rightarrow 0$ as $n \rightarrow \infty$.

Proof sketch. There is a relatively elementary proof based on Lemma 6.5.

7.4. Proof of Theorem 7.1. Let $\widetilde{S}$ be a weak solution of Problem 2.1, and let $\left(S_{*, n}^{-}, S_{n}, S_{*, n}^{+}\right)_{n=1}^{\infty}$ be a corresponding approximating sequence (of solutions of Problem 3.9), corresponding to the positive null sequence $\left(\varepsilon_{n}\right)_{n=1}^{\infty}$. For the purpose of obtaining a contradiction, assume there exists a point $x_{0} \in \widetilde{S}$ such that $e\left(x_{0}\right):=F\left(x_{0}, \widetilde{Q}^{-}\left(x_{0}\right), \widetilde{Q}^{+}\left(x_{0}\right)\right)>0$. Then there exist positive values $\mu_{0}^{ \pm}$such that $\pm\left(\widetilde{Q}^{ \pm}\left(x_{0}\right)-\mu_{0}^{ \pm}\right)>0$ and $F\left(x_{0}, \mu_{0}^{-}, \mu_{0}^{+}\right) \geq\left(e\left(x_{0}\right) / 2\right)$. In terms of notation introduced in the proof of Proposition 7.2, we have $\pm\left(\widetilde{Q}^{ \pm}\left(\tilde{p}^{ \pm}(t)\right)-\widetilde{Q}^{ \pm}\left(x_{0}\right)\right) \uparrow 0$ as $t \downarrow 0$ due to Theorem 6.2(f). Therefore, $\pm\left(\widetilde{Q}^{ \pm}\left(\tilde{p}^{ \pm}\left(t_{0}\right)\right)-\mu_{0}^{ \pm}\right)>0$ for a fixed, sufficiently small value $t_{0}>0$. By the continuity of $\widetilde{Q}^{ \pm}(x)$, we have $\pm\left(\widetilde{Q}^{ \pm}(x)-\mu_{0}^{ \pm}\right)>0$ in $B_{\delta}\left(\tilde{p}^{ \pm}\left(t_{0}\right)\right) \subset \widetilde{\Omega}^{ \pm}$for a sufficiently small value $\delta=\delta\left(t_{0}\right)>0$. Since $U_{n}^{ \pm}(x) \rightarrow \widetilde{U}^{ \pm}(x)$ as $n \rightarrow \infty$ uniformly in compact subsets of $\widetilde{\Omega}^{ \pm}$, it follows that $\nabla U_{n}^{ \pm}(x) \rightarrow \nabla \widetilde{U}^{ \pm}(x)$ as $n \rightarrow \infty$ uniformly in compact subsets of $\widetilde{\Omega}^{ \pm}$. Therefore, $\pm\left(Q_{n}^{ \pm}(x)-\mu_{0}^{ \pm}\right)>0$ in $B_{\delta}\left(\tilde{p}^{ \pm}\left(t_{0}\right)\right)$, provided that $n$ is sufficiently large. Thus,

$$
F\left(x_{0}, Q_{n}^{-}\left(x^{-}\right), Q_{n}^{+}\left(x^{+}\right)\right) \geq\left(e\left(x_{0}\right) / 2\right)
$$

for sufficiently large $n \in \mathbb{N}$, and for all $x^{ \pm} \in B_{\delta}\left(\tilde{p}^{ \pm}\left(t_{0}\right)\right)$. On the other hand, by Lemma 6.10 , there exist sequences of points $\left(x_{n}^{ \pm}\right)$such that $x_{n}^{ \pm} \in \Omega_{n}^{ \pm}$for all $n \in \mathbb{N}$, and such that $x_{n}^{ \pm} \rightarrow x_{0}$ and $F\left(x_{0}, Q_{n}^{-}\left(x_{n}^{-}\right), Q_{n}^{+}\left(x_{n}^{+}\right)\right) \leq \zeta(n)$, both as $n \rightarrow \infty$. For each $n \in \mathbb{N}$, let $\gamma_{n}^{ \pm}$denote the arc of steepest ascent of $U_{n}^{ \pm}(\cdot)$ which passes through the point $x_{n}^{ \pm}$. It follows from Lemma 6.7 and Proposition 7.2 that $\gamma_{n}^{ \pm} \cap B_{\delta}\left(\tilde{p}^{ \pm}\left(t_{0}\right)\right) \neq$ $\varnothing$ for sufficiently large $n \in \mathbb{N}$. Moreover, since $\pm Q_{n}^{ \pm}(x)$ is monotone decreasing with increasing $U_{n}^{ \pm}$on $\gamma_{n}^{ \pm}$(by Lemma 3.4(d)), we have that $\pm\left(Q_{n}^{ \pm}\left(x^{ \pm}\right)-Q_{n}^{ \pm}\left(x_{n}^{ \pm}\right)\right) \leq 0$ for sufficiently large $n \in \mathbb{N}$, and for all $x^{ \pm} \in \gamma_{n}^{ \pm} \cap B_{\delta}\left(\tilde{p}^{ \pm}\left(t_{0}\right)\right)$. In view of Assumption $2.2(\mathrm{a})$, we have

$$
F\left(x_{0}, Q_{n}^{-}\left(x^{-}\right), Q_{n}^{+}\left(x^{+}\right)\right) \leq \zeta(n)
$$

for sufficiently large $n \in \mathbb{N}$, and for all $x^{ \pm} \in \gamma_{n}^{ \pm} \cap B_{\delta}\left(\tilde{p}^{ \pm}\left(t_{0}\right)\right)$. Clearly, (7.17) contradicts (7.16) for sufficiently large $n \in \mathbb{N}$. This contradiction proves the assertion.

\section{A PROPERTY OF BOUNDARY DERIVATIVES OF WEAK SOLUTIONS}

8.1. Theorem. Let $\widetilde{S} \in \mathbb{X}$ denote a weak solution of Problem 2.1, where Assumptions $2.2(\mathrm{a})-(\mathrm{d})$ hold. Let $F^{ \pm}(t):\left[\mathfrak{C}_{0}, \mathfrak{C}_{1}\right] \rightarrow \mathbb{R}_{+}$denote positive $C^{2}$-functions such that $d F^{ \pm}(t) / d t>0$ and $d^{2} F^{-}(t) / d t^{2} \geq 0$ (where $\mathfrak{C}_{0}, \mathfrak{C}_{1}$ appear in Lemma 6.2(a)). Then, for any given point $x_{0} \in \widetilde{S}$ and for any given value $\eta>0$, we have

$$
\pm\left(1 /\left|\tilde{s}_{n}\right|\right) \int_{\tilde{s}_{n}}\left(F^{ \pm}\left(\widetilde{Q}^{ \pm}(x)\right)-F^{ \pm}\left(\widetilde{Q}^{ \pm}\left(x_{0}\right)\right)\right) d \sigma<\eta
$$

for all sufficiently large $n \in \mathbb{N}$, where $\tilde{s}_{n}=\left\{x \in \widetilde{S}: \tilde{\nu}(x) \cdot \tilde{\nu}\left(x_{0}\right)>0\right.$ and $d(x, \mathcal{L})<$ $\left.2^{-n}\right\}, \mathcal{L}=\mathcal{L}\left(x_{0}\right)=\left\{x_{0}+\tilde{\nu}\left(x_{0}\right) t: t \in \mathbb{R}\right\},\left|\tilde{s}_{n}\right|$ denotes the $(N-1)$-dimensional 
surface area of $\tilde{s}_{n}$, and $d \sigma$ denotes the differential area of an $(N-1)$-dimensional surface.

8.2. Proposition. Let $\widetilde{S} \in \mathbb{X}$ denote a weak solution of Problem 2.1, where Assumptions $2.2(\mathrm{a})-(\mathrm{d})$ hold. Let $G^{ \pm}(t): \mathbb{R}_{+} \rightarrow \mathbb{R}_{+}$denote $C^{2}$-functions such that $d G^{ \pm}(t) / d t>0, d^{2} G^{+}(t) / d t^{2} \geq 0$, and $d\left(t d G^{-}(t) / d t\right) / d t \leq 0$, all relative to the $t$-interval $\left[\mathfrak{C}_{0}, \mathfrak{C}_{1}\right]$. Then, for any given point $x_{0} \in \widetilde{S}$ and for any given value $\eta>0$, we have

$$
\pm\left(1 /\left|\tilde{s}_{n}\right|\right) \int_{\tilde{s}_{n}}\left(G^{ \pm}\left(\widetilde{Q}^{ \pm}(x)\right)-G^{ \pm}\left(\widetilde{Q}^{ \pm}\left(x_{0}\right)\right)\right) \widetilde{Q}^{ \pm}(x) d \sigma<\eta
$$

(in the notation of Theorem 8.1) for all sufficiently large $n \in \mathbb{N}$.

8.3. Definition. Given a weak solution $\widetilde{S}$ of Problem 2.1 and a point $x_{0} \in \widetilde{S}$, we define the blow-up functions $\widetilde{U}_{n}^{ \pm}(x):=2^{n} \widetilde{U}^{ \pm}\left(x_{0}+2^{-n}\left(x-x_{0}\right)\right), n \in \mathbb{N}$, in the blow-up domains $\widetilde{\Omega}_{n}^{ \pm}:=\left\{x_{0}+2^{n}\left(x-x_{0}\right): x \in \widetilde{\Omega}^{ \pm}\right\}$, with common boundary $\widetilde{S}_{n}:=\left\{x_{0}+2^{n}\left(x-x_{0}\right): x \in \widetilde{S}\right\}$ (see [15], §4). Observe that the differentiability of the functions $\widetilde{U}^{ \pm}(x)$ at the point $x_{0} \in \widetilde{S}$ (see Theorem $6.2(\mathrm{c})$ ) is equivalent to the property that

$$
\widetilde{U}_{n}^{ \pm}(x)= \pm \lambda^{ \pm}\left(x_{0}\right) \tilde{\nu}\left(x_{0}\right) \cdot\left(x-x_{0}\right)+2^{n} o\left(2^{-n}\left|x-x_{0}\right|\right)
$$

relative to the set $C l\left(\widetilde{\Omega}_{n}^{ \pm}\right)$.

8.4. Proof of Proposition 8.2. This proof of Proposition 8.2 is closely related to the proof of [9], Lemma 5.4, which is perhaps the most important special case. We will prove the assertion in the "+" case in detail and then remark briefly on the proof of the "-" case. The proof is expressed in the blow-up notation of Definition 8.3. Since the entire proof concerns a specified point $x_{0}$ in a fixed weak solution $\widetilde{S}$ of Problem 2.1, and is also restricted to the "+" case, we simplify the notation by deleting the tilde and the plus sign, so that $\widetilde{U}_{n}^{+}(x), \widetilde{\Omega}_{n}^{+}, \tilde{S}_{n}, G^{+}\left(Q_{n}^{+}\right)$, and $\lambda^{+}\left(x_{0}\right)$ become $U_{n}(x), \Omega_{n}, S_{n}, G\left(Q_{n}\right)$, and $\lambda_{0}$. We also choose Cartesian coordinates such that $x_{0}=0, \tilde{\nu}_{0}=\nu_{0}:=(0, \ldots, 0,1)$, and $x=(y, z)=\left(y_{1}, \ldots, y_{N-1}, z\right)$, and let $\mathfrak{D}=\left\{y \in \mathbb{R}^{N-1}:|y| \leq 1\right\}$. Let $W(y): \mathfrak{D} \rightarrow \mathbb{R}$ denote a convex, radially symmetric, $C^{2}$-function of $y$ such that $W(0)=-2 \lambda_{0}, \nabla_{y} W(0)=0,\left|\nabla_{y} W(y)\right| \geq 3 \mathfrak{C}_{1}$ for $\left(1-\left(\lambda_{0} / 3 \mathfrak{C}_{1}\right)\right) \leq|y| \leq 1$, and $W(y)=0$ for $|y|=1$. Our proof is based on Green's second identity, in the form

$$
\int_{\Omega_{\delta, \varepsilon, n}}\left(\psi_{n} \Delta \phi_{n}-\phi_{n} \Delta \psi_{n}\right) d x=\int_{\partial \Omega_{\delta, \varepsilon, n}}\left(\psi_{n} \frac{\partial}{\partial \nu} \phi_{n}-\phi_{n} \frac{\partial}{\partial \nu} \psi_{n}\right) d \sigma
$$

where we define $\phi_{n}(x):=G\left(Q_{n}(x)\right)-G\left(\lambda_{0}\right), \psi_{n}:=\left(U_{n}+W\right)$, and

$$
\Omega_{\delta, \varepsilon, n}:=\left\{x \in \Omega_{n}: U_{n}>\delta, z<\varepsilon, U_{n}+W<0\right\}
$$

for all small $\delta, \varepsilon>0$ and large $n \in \mathbb{N}$ (and where $\nu$ denotes the exterior normal direction on $\left.\partial \Omega_{\delta, \varepsilon, n}\right)$. For $(\delta / \varepsilon)$ sufficiently small, a partition of $\partial \Omega_{\delta, \varepsilon, n}$ into disjoint surfaces is given by $\partial \Omega_{\delta, \varepsilon, n}=S_{\delta, n} \cup L_{\varepsilon, n} \cup \Sigma_{\delta, \varepsilon, n}$, where $S_{\delta, n}=\left\{U_{n}=\delta, U_{n}+W \leq\right.$ $0\}, L_{\varepsilon, n}=\left\{z=\varepsilon, U_{n}+W \leq 0\right\}$, and $\Sigma_{\delta, \varepsilon, n}=\left\{U_{n}>\delta, z<\varepsilon, U_{n}+W=0\right\}$. Now $\Delta \phi_{n}=G^{\prime}\left(Q_{n}\right) \Delta Q_{n}+G^{\prime \prime}\left(Q_{n}\right)\left|\nabla Q_{n}\right|^{2} \geq 0$ and $\psi_{n}<0$ in $\Omega_{\delta, \varepsilon, n}$ (see Lemma 3.4(e)). Therefore $\psi_{n} \Delta \phi_{n} \leq 0$ in $\Omega_{\delta, \varepsilon, n}$. Also, $\sup \left\{\left|\phi_{n}(x) \Delta \psi_{n}\right|: x \in \Omega_{\delta, \varepsilon, n}\right\} \leq M$ (= a constant), uniformly for all small $\delta, \varepsilon>0$ and large $n \in \mathbb{N}$, because $\Delta \psi_{n}=\Delta W \geq 0$ and because $\phi_{n}(x):=G\left(Q_{n}(x)\right)-G\left(\lambda_{0}\right)$ is uniformly bounded, due to Theorem $6.2(\mathrm{a})$ and the fact that $Q_{n}(x)=Q\left(2^{-n} x\right)$. Finally, we have $\left|\Omega_{\delta, \varepsilon, n}\right| \leq O(\varepsilon)+\zeta(n)$ 
(independent of $\delta>0$ ) because $\widetilde{S}$ is a uniformly $C^{1}$-surface, where $|\cdot|$ denotes Euclidean volume. Thus

$$
\int_{\Omega_{\delta, \varepsilon, n}}\left(\psi_{n} \Delta \phi_{n}-\phi_{n} \Delta \psi_{n}\right) d x \leq O(\varepsilon)+\zeta(n)
$$

as $\delta, \varepsilon \rightarrow 0+,(\delta / \varepsilon) \rightarrow 0+$, and $n \rightarrow \infty$. Now $\max \left\{\left|\psi_{n}\right|,\left|\nabla \psi_{n}\right|: x \in L_{\varepsilon, n}\right\}$ is uniformly bounded for fixed $\varepsilon>0$ as $n \rightarrow \infty$, due to Theorem 6.2(a) and the fact that $\nabla U_{n}(x)=\nabla U\left(2^{-n} x\right)$. Now by eq. (8.3), we have that $\left(U_{n}(x)-\lambda_{0} \nu_{0} \cdot x\right) \rightarrow 0$ as $n \rightarrow \infty$, uniformly in compact subsets of $\{z>0\}$. Therefore $\nabla U_{n}(x) \rightarrow \lambda_{0} \nu_{0}$ and $\nabla\left(\partial U_{n}(x) / \partial \nu_{0}\right) \rightarrow 0$, both uniformly in compact subsets of $\{z>0\}$. Since

$$
\partial \phi_{n}(x) / \partial \nu_{0}=\left(G^{\prime}\left(Q_{n}\right) / Q_{n}\right)\left(\nabla U_{n} \cdot \nabla\left(\partial U_{n} / \partial \nu_{0}\right)\right),
$$

it follows that $\max \left\{\left|\phi_{n}(x)\right|,\left|\partial \phi_{n}(x) / \partial \nu_{0}\right|: x \in L_{\varepsilon, n}\right\} \rightarrow 0$ as $n \rightarrow \infty$ for any fixed $\varepsilon>0$. Thus,

$$
\int_{L_{\varepsilon, n}}\left(\psi_{n} \frac{\partial}{\partial \nu} \phi_{n}-\phi_{n} \frac{\partial}{\partial \nu} \psi_{n}\right) d \sigma=\zeta_{\varepsilon}(n),
$$

where for each $\varepsilon>0, \zeta_{\varepsilon}(n)$ denotes a function such that $\zeta_{\varepsilon}(n) \rightarrow 0$ as $n \rightarrow \infty$. Due to the fact that $\left|\nabla U_{n}\right| \leq \mathfrak{C}_{1}$ and $|\nabla W| \geq 3 \mathfrak{C}_{1}$ for $\left(1-\left(\lambda_{0} / 3 \mathfrak{C}_{1}\right)\right) \leq|y| \leq 1$, we have that $\nu \cdot \nu_{r} \geq 1 / 2$ for $\left(1-\left(\lambda_{0} / 3 \mathfrak{C}_{1}\right)\right) \leq|y| \leq 1$, where $\nu=\nabla\left(U_{n}+W\right) /\left|\nabla\left(U_{n}+W\right)\right|$ and $\nu_{r}=\nabla W /|\nabla W|=y /|y|$. Since $\nu$ is the outer normal to the surface $\Sigma_{\delta, \varepsilon, n}$ at each of its points, it follows that the area of $\Sigma_{\delta, \varepsilon, n}$ is bounded by $O(\varepsilon)+\zeta(n)$ (independent of $\delta>0$ ) as $\varepsilon \rightarrow 0+$. Also, $\psi_{n}=0$ on $\Sigma_{\delta, \varepsilon, n}$, and the functions $\phi_{n}:=G\left(Q_{n}\right)-G(\lambda)$ and $\nabla \psi_{n}:=\nabla\left(U_{n}+W\right)$ both remain uniformly bounded in a uniform neighborhood of $S_{n}$ as $n \rightarrow \infty$. In view of these facts, we have

$$
\int_{\Sigma_{\delta, \varepsilon, n}}\left(\psi_{n} \frac{\partial}{\partial \nu} \phi_{n}-\varphi_{n} \frac{\partial}{\partial \nu} \psi_{n}\right) d \sigma=-\int_{\Sigma_{\delta, \varepsilon, n}} \phi_{n} \frac{\partial}{\partial \nu} \psi_{n} d \sigma=O(\varepsilon)+\zeta(n)
$$

as $\delta, \varepsilon \rightarrow 0+$, independent of $n \in \mathbb{N}$. By substituting (8.5), (8.6), and (8.7) into (8.4), one obtains

$$
\int_{S_{\delta, n}}\left(\phi_{n} \frac{\partial}{\partial \nu_{n}} \psi_{n}-\psi_{n} \frac{\partial}{\partial \nu_{n}} \phi_{n}\right) d \sigma \leq O(\varepsilon)+\zeta_{\varepsilon}(n)
$$

(with $\nu_{n}=\nabla U_{n} / Q_{n}$ on $S_{\delta, n}$ ). This is equivalent (using the definitions of $\phi_{n}$ and $\left.\psi_{n}\right)$ to

$$
\begin{aligned}
\int_{S_{\delta, n}} & \phi_{n}(x)\left(Q_{n}+\left(\partial W(y) / \partial \nu_{n}\right)\right) d \sigma \\
\quad \leq & \int_{S_{\delta, n}}|\delta+W| G^{\prime}\left(Q_{n}\right)\left|\left(\partial Q_{n} / \partial \nu_{n}\right)\right| d \sigma+O(\varepsilon)+\zeta_{\varepsilon}(n) .
\end{aligned}
$$

Now $|\delta+W| \leq M$ and $\mathfrak{C}_{0} \leq Q_{n} \leq \mathfrak{C}_{1}$ on $S_{\delta, n}$, both uniformly as $n \rightarrow \infty$ and $\delta, \varepsilon \rightarrow 0+$. Also, it follows from Lemmas 6.4 and 6.5 that

$$
\max \left\{\left|\nu_{n}(x)-\nu_{0}\right|: x \in S_{\delta, n}\right\} \leq \zeta(n)+z(\delta)
$$

as $n \rightarrow \infty$ and $\delta \rightarrow 0+$. Therefore $\max \left\{\left|\nabla W(y) \cdot \nu_{n}(x)\right|: x \in S_{\delta, n}\right\} \leq \zeta(n)+z(\delta)$ as $n \rightarrow \infty$ and $\delta \rightarrow 0+$, since $\nabla W(y)$ has no component in the $\nu_{0}$-direction. Therefore, (8.8) implies

$$
\int_{S_{\delta, n}} \phi_{n}(x) Q_{n}(x) d \sigma \leq M \int_{S_{\delta, n}}\left(\left|\partial^{2} U_{n} / \partial \nu_{n}^{2}\right| / Q_{n}\right) d \sigma+z(\delta)+O(\varepsilon)+\zeta_{\varepsilon}(n)
$$


for a uniform constant $M$. For sufficiently large $n \in \mathbb{N}$ and sufficiently small $\delta>0, S_{\delta, n}$ is the graph of a smooth function $z=S_{\delta, n}(y): \mathfrak{D}_{\delta, n} \rightarrow \mathbb{R}$. For this representation, we have

$$
\left(\partial^{2} U_{n}(x) / \partial \nu_{n}^{2}\right) / Q_{n}(x)=\nabla_{y} \cdot\left(\nabla_{y} S_{\delta, n}(y) /\left[1+\left|\nabla_{y} S_{\delta, n}(y)\right|^{2}\right]^{1 / 2}\right),
$$

where both sides represent $(N-1)$ times the mean curvature of the surface $S_{\delta, n}$ at $x=(y, z)=\left(y, S_{\delta, n}(y)\right) \in S_{\delta, n}$. By substituting (8.11) into the second integral of (8.10), estimating $d \sigma / d y=\left(1+\left|\nabla S_{\delta, n}(y)\right|^{2}\right)^{1 / 2}$ by a constant, and applying the divergence theorem, one obtains

$$
\int_{S_{\delta, n}} \phi_{n}(x) Q_{n}(x) d \sigma \leq M \int_{\partial \mathfrak{D}_{\delta, n}}\left|\nabla S_{\delta, n}(y)\right| d \sigma+z(\delta)+O(\varepsilon)+\zeta_{\varepsilon}(n),
$$

where the integrand of the second integral is uniformly bounded by $\zeta(n)+z(\delta)$, due to $(8.9)$, and where $d \sigma$ in the second integral refers to $(N-2)$-dimensional surface area. In the limit as $\delta \rightarrow 0+$, we obtain

$$
\int_{C_{n}} \phi_{n}(x) Q_{n}(x) d \sigma \leq O(\varepsilon)+\zeta_{\varepsilon}(n)
$$

where $C_{n}=S_{n} \cap\{x=(y, z):|y|<1,|z|<1\}$. This implies the assertion in the "+" case. Finally, for the corresponding proof in the "-" case, one again simplifies the notation so that $\widetilde{U}_{n}^{-}(x), \widetilde{\Omega}_{n}^{-}, \widetilde{S}_{n}, G^{-}\left(Q_{n}^{-}\right)$and $\lambda^{-}\left(x_{0}\right)$ become $U_{n}(x), \Omega_{n}, S_{n}, G\left(Q_{n}\right)$, and $\lambda_{0}$. The proof again is again based on Green's second identity (8.4), where $\phi_{n}(x):=G\left(Q_{n}(x)\right)-G\left(\lambda_{0}\right), \psi_{n}:=\left(U_{n}+W\right), \Omega_{\delta, \varepsilon, n}=\left\{x \in \Omega_{n}: U_{n}(x)>\delta, z>\right.$ $\left.-\varepsilon, U_{n}(x)+W(y)<0\right\}$, and $W(y)$ has the same properties assumed in the "+" case. Under the assumption that $d(t d G(t) / d t) / d t \leq 0$, it follows from the convexity of the level surfaces of $U_{n}$ that $\Delta \phi_{n}(x) \leq 0$ throughout $\Omega_{n}$ (see Lemma 3.4(e)). Continuing as in the "+" case, one can show (in the "-" case) that

$$
\begin{aligned}
& \int_{S_{\delta, n}}\left(G\left(Q_{n}(x)\right)-G\left(\lambda_{0}\right)\right) Q_{n}(x) d \sigma \\
& \quad \geq-M \int_{S_{\delta, n}}\left(\left|\partial^{2} U_{n} / \partial \nu_{n}^{2}\right| / Q_{n}\right) d \sigma-z(\delta)-O(\varepsilon)-\zeta_{\varepsilon}(n),
\end{aligned}
$$

where $S_{\delta, n}=\left\{x \in \Omega_{n}: U_{n}(x)=\delta, U_{n}(x)+W(y)<0\right\}$. Then the assertion follows by the steps given above.

8.5. Corollary. Let $\widetilde{S} \in \mathbb{X}$ denote a weak solution of Problem 2.1, where Assumptions $2.2(\mathrm{a})-(\mathrm{d})$ hold. Let the function $F^{+}(t):\left[\mathfrak{C}_{0}, \mathfrak{C}_{1}\right] \rightarrow \mathbb{R}$ be defined such that $F^{+}(t)=H^{+}\left(G^{+}(t)\right)$, where the $C^{2}$-function $G^{+}(t):\left[\mathfrak{C}_{0}, \mathfrak{C}_{1}\right] \rightarrow \mathbb{R}$ is chosen such that $d G^{+}(t) / d t>0$, and $d^{2} G^{+}(t) / d t^{2} \geq 0$ for $t \in\left[\mathfrak{C}_{0}, \mathfrak{C}_{1}\right]$, and where the $C^{2}$-function $H^{+}(\tau): G^{+}\left(\left[\mathfrak{C}_{0}, \mathfrak{C}_{1}\right]\right) \rightarrow \mathbb{R}$ is chosen such that $d H^{+}(\tau) / d \tau>0$ and $d^{2} H^{+}(\tau) / d \tau^{2} \leq 0$. Then, for any given point $x_{0} \in \widetilde{S}$ and value $\eta>0$, we have

$$
\left(1 /\left|\tilde{s}_{n}\right|\right) \int_{\tilde{s}_{n}}\left(F^{+}\left(\widetilde{Q}^{+}(x)\right)-F^{+}\left(\widetilde{Q}^{+}\left(x_{0}\right)\right)\right) d \sigma<\eta
$$

(in terms of notation from Theorem 8.1) for all sufficiently large $n \in \mathbb{N}$. 
Proof. Since $\left(t-t_{0}\right)\left(G^{+}(t)-G^{+}\left(t_{0}\right)\right) \geq 0$ for all $t_{0}, t>0$, it follows from Proposition 8.2 (eq. (8.2) that for any $\eta>0$, we have

$$
\begin{aligned}
\int_{\tilde{s}_{n}}\left(G^{+}\left(\widetilde{Q}^{+}(x)\right)-G^{+}\left(\widetilde{Q}^{+}\left(x_{0}\right)\right)\right) d \sigma \\
\quad \leq \int_{\tilde{s}_{n}}\left(G^{+}\left(\widetilde{Q}^{+}(x)\right)-G^{+}\left(\widetilde{Q}^{+}\left(x_{0}\right)\right)\right)\left[\widetilde{Q}^{+}(x) / \widetilde{Q}^{+}\left(x_{0}\right)\right] d \sigma<\left(\left|\tilde{s}_{n}\right| \eta / \widetilde{Q}^{+}\left(x_{0}\right)\right)
\end{aligned}
$$

for sufficiently large $n \in \mathbb{N}$. It follows from the assumed properties of $H^{+}(\tau)$ that for any $\tau_{0} \in G^{+}\left(\left[\mathfrak{C}_{0}, \mathfrak{C}_{1}\right]\right)$, we have

$$
\left(H^{+}(\tau)-H^{+}\left(\tau_{0}\right)\right) \leq\left(d H^{+}\left(\tau_{0}\right) / d \tau\right)\left(\tau-\tau_{0}\right)
$$

for all $\tau \in G^{+}\left(\left[\mathfrak{C}_{0}, \mathfrak{C}_{1}\right]\right)$. It follows that for any $t_{0} \in\left[\mathfrak{C}_{0}, \mathfrak{C}_{1}\right]$, one has

$$
\left(F^{+}(t)-F^{+}\left(t_{0}\right)\right) \leq\left(d H^{+}\left(G^{+}\left(t_{0}\right)\right) / d \tau\right)\left(G^{+}(t)-G^{+}\left(t_{0}\right)\right)
$$

for all $t \in\left[\mathfrak{C}_{0}, \mathfrak{C}_{1}\right]$, where $d H^{+}\left(G^{+}\left(t_{0}\right)\right) / d \tau>0$. In view of this inequality, the assertion follows from eq. (8.14).

8.6. Proof of Theorem 8.1 in the "+" case. It suffices to express the functions $F^{+}(t):\left[\mathfrak{C}_{0}, \mathfrak{C}_{1}\right] \rightarrow \mathbb{R}_{+}$in the form required by Corollary 8.5. A straightforward calculation shows that this is accomplished by defining $G^{+}(t)=\left(F^{+}(t)\right)^{\alpha}$ and $H^{+}(\tau)=\tau^{(1 / \alpha)}$, where $\alpha>0$ is sufficiently large.

8.7. Proof of Theorem 8.1 in the "-" case. Given $t_{0} \in\left[\mathfrak{C}_{0}, \mathfrak{C}_{1}\right]$ and the function $F^{-}(t):\left[\mathfrak{C}_{0}, \mathfrak{C}_{1}\right] \rightarrow \mathbb{R}$, it suffices (in view of Proposition 8.2), to choose an increasing $C^{2}$-function $G^{-}(t):\left[\mathfrak{C}_{0}, \mathfrak{C}_{1}\right] \rightarrow \mathbb{R}$ such that $d\left(t d G^{-}(t) / d t\right) / d t \leq 0$ and

$$
\phi(t):=\left(F^{-}(t)-F^{-}\left(t_{0}\right)\right)-C_{0} t\left(G^{-}(t)-G^{-}\left(t_{0}\right)\right) \geq 0
$$

for all $t \in\left[\mathfrak{C}_{0}, \mathfrak{C}_{1}\right]$, where $C_{0}:=\left(\left(d F^{-}\left(t_{0}\right) / d t\right) / t_{0}\left(d G^{-}\left(t_{0}\right) / d t\right)\right)>0$. Since $\phi\left(t_{0}\right)=$ $\phi^{\prime}\left(t_{0}\right)=0$, it suffices to have $\phi^{\prime \prime}(t):=\left(d^{2}\left(F^{-}(t)-C_{0} t G^{-}(t)\right) / d t^{2}\right) \geq 0$ for all $t \in\left[\mathfrak{C}_{0}, \mathfrak{C}_{1}\right]$. For the case where $d^{2} F^{-}(t) / d t^{2} \geq 0$, the requirements are all satisfied by making the choice $G^{-}(t)=-t^{\alpha}$, where $\alpha \leq-1$.

Remark. The factor " $2^{n}$ " in the statement of [9], Lemma 5.4 should be " $2^{(m-1) n}$ ", where $m$ denotes dimension. In the briefly outlined proof of the "-" case (on p. 221 , line 6$)$, one should define $\phi_{n}(x)=\left((1 / \lambda)-\left(1 /\left|\nabla U_{n}\right|\right)\right)$, so that the first integral in the equation on line 11 becomes $\int_{\Gamma_{\delta, n}}\left(\left(\left|\nabla U_{n}\right|-\lambda\right) / \lambda\right) d s$.

$$
\text { 9. Proof that } F^{-}\left(x_{0}, \widetilde{Q}^{-}\left(x_{0}\right)\right) \leq F^{+}\left(x_{0}, \widetilde{Q}^{+}\left(x_{0}\right)\right) \text { FOR ALL } x_{0} \in \widetilde{S}
$$

9.1. Theorem. Let $\widetilde{S} \in \mathbb{X}$ denote a weak solution of Problem 2.1, where Assumptions 2.2(a)-(e) all apply. Then, in the notation of $\S 6.1$, we have

$$
F^{-}\left(x_{0}, \widetilde{Q}^{-}\left(x_{0}\right)\right) \leq F^{+}\left(x_{0}, \widetilde{Q}^{+}\left(x_{0}\right)\right)
$$

for all $x_{0} \in \widetilde{S}$

9.2. Lemma. Under Assumptions $2.2(\mathrm{a})-(\mathrm{e})$, let $\widetilde{S} \in \mathbb{X}$ denote a weak solution of Problem 2.1 such that $F^{+}\left(x_{0}, \widetilde{Q}^{+}\left(x_{0}\right)\right)<F^{-}\left(x_{0}, \widetilde{Q}^{-}\left(x_{0}\right)\right)$ at a point $x_{0} \in \widetilde{S}$. Then

$$
\int_{\tilde{s}_{n}} F^{+}\left(x, \widetilde{Q}^{+}(x)\right) d \sigma<\int_{\tilde{s}_{n}} F^{-}\left(x, \widetilde{Q}^{-}(x)\right) d \sigma-2\left|\tilde{s}_{n}\right| \eta_{0}
$$


for all sufficiently large $n \in \mathbb{N}$ (i.e. $n \geq n_{0}$ ), where

$$
\eta_{0}=(1 / 6)\left(F^{-}\left(x_{0}, \widetilde{Q}^{-}\left(x_{0}\right)\right)-F^{+}\left(x_{0}, \widetilde{Q}^{+}\left(x_{0}\right)\right)\right)>0,
$$

$\tilde{s}_{n}=\left\{x \in \widetilde{S}: \tilde{\nu}(x) \cdot \tilde{\nu}\left(x_{0}\right)>0\right.$ and $\left.d(x, \mathcal{L})<2^{-n}\right\}, \mathcal{L}=\mathcal{L}\left(x_{0}\right)=\left\{x_{0}+\tilde{\nu}\left(x_{0}\right) t:\right.$ $t \in \mathbb{R}\},\left|\tilde{s}_{n}\right|$ denotes the corresponding $(N-1)$-dimensional surface area, and $d \sigma$ denotes the $(N-1)$-dimensional differential area of $\widetilde{S}$. Moreover, if $x_{\beta}^{ \pm}$denotes the projection of $x \in \widetilde{S}$ onto the surface $\widetilde{S}_{\beta}^{ \pm}:=\left\{\widetilde{U}^{ \pm}(x)=\beta\right\}$ along a curve of steepest ascent of $\widetilde{U}^{ \pm}$, then for each fixed $n \geq n_{0}$, we have

$$
\int_{\tilde{s}_{n, \beta}^{+}} F^{+}\left(x, \widetilde{Q}^{+}\left(x_{\beta}^{+}\right)\right) d \sigma_{\beta}^{+}<\int_{\tilde{s}_{n, \beta}^{-}} F^{-}\left(x, \widetilde{Q}^{-}\left(x_{\beta}^{-}\right)\right) d \sigma_{\beta}^{-}-\left|\tilde{s}_{n}\right| \eta_{0}
$$

for all $\beta \in\left(0, \beta_{0}(n)\right)$, where $\tilde{s}_{n, \beta}^{ \pm}=\left\{x_{\beta}^{ \pm}: x \in \tilde{s}_{n}\right\} \subset \widetilde{S}_{\beta}^{ \pm}, \beta_{0}(n) \in(0,1)$ is sufficiently small, and $d \sigma_{\beta}^{ \pm}$denotes $(N-1)$-dimensional differential surface area of $\widetilde{S}_{\beta}^{ \pm}$.

Proof. We remark that the differential areas of the surfaces $\widetilde{S}$ and $\widetilde{S}_{\beta}^{ \pm}$at points $x \in \widetilde{S}$ and $x_{\beta}^{ \pm} \in \widetilde{S}_{\beta}^{ \pm}$on the same curve of steepest ascent of the function $\widetilde{U}^{ \pm}$are related by the equation $\widetilde{Q}^{ \pm}(x) d \sigma=\widetilde{Q}^{ \pm}\left(x_{\beta}^{ \pm}\right) d \sigma_{\beta}^{ \pm}$(see [9], Lemma 4.4). For any fixed $x_{0} \in \widetilde{S}$, it follows by combining the \pm cases of Theorem 8.1 (with $\eta=\eta_{0}$ ) that

$$
\int_{\tilde{s}_{n}} F^{+}\left(x_{0}, \widetilde{Q}^{+}(x)\right) d \sigma<\int_{\tilde{s}_{n}} F^{-}\left(x_{0}, \widetilde{Q}^{-}(x)\right) d \sigma-4\left|\tilde{s}_{n}\right| \eta_{0}
$$

for sufficiently large $n \in \mathbb{N}$. In view of Theorem 6.2(a), it follows from the assumed smoothness of the functions $F^{ \pm}(x, t)$ that there exists a constant $L$ such that

$$
\left|F^{ \pm}\left(x, \widetilde{Q}^{ \pm}(x)\right)-F^{ \pm}\left(x_{0}, \widetilde{Q}^{ \pm}(x)\right)\right| \leq L\left|x-x_{0}\right|,
$$

uniformly for all $x \in \widetilde{S}$. In view of (9.4) and (9.5), the inequality (9.2) follows from the obvious fact that $\left(1 /\left|\tilde{s}_{n}\right|\right) \int_{\tilde{s}_{n}}\left|x-x_{0}\right| d \sigma \rightarrow 0$ as $n \rightarrow \infty$. Also,

$$
\pm\left[F^{ \pm}\left(x, \widetilde{Q}^{ \pm}\left(x_{\beta}^{ \pm}\right)\right)-F^{ \pm}\left(x, \widetilde{Q}^{ \pm}(x)\right)\right] \leq 0,
$$

as follows from the assumed monotonicity (in $t$ ) of the functions $F^{ \pm}(x, t)$, and the monotonicity of $\widetilde{Q}^{ \pm}(x)$ on curves of steepest ascent of $\widetilde{U}^{ \pm}$(Lemma 3.4(d)). Therefore,

$$
\pm \int_{\tilde{s}_{n, \beta}^{ \pm}} F^{ \pm}\left(x, \widetilde{Q}^{ \pm}\left(x_{\beta}^{ \pm}\right)\right) d \sigma_{\beta}^{ \pm} \leq \pm \int_{\widetilde{s}_{n}} F^{ \pm}\left(x, \widetilde{Q}^{ \pm}(x)\right)\left(\widetilde{Q}^{ \pm}(x) / \widetilde{Q}^{ \pm}\left(x_{\beta}^{ \pm}\right)\right) d \sigma .
$$

On the other hand, since $\left(\widetilde{Q}^{ \pm}(x) / \widetilde{Q}^{ \pm}\left(x_{\beta}^{ \pm}\right)\right) \rightarrow 1$ for each $x \in \widetilde{S}$ as $\beta \downarrow 0$ (by Theorem 6.2(f)), the Lebesgue dominated convergence theorem implies that

$$
\int_{\widetilde{s}_{n}} F^{ \pm}\left(x, \widetilde{Q}^{ \pm}(x)\right)\left(\widetilde{Q}^{ \pm}(x) / \widetilde{Q}^{ \pm}\left(x_{\beta}^{ \pm}\right)\right) d \sigma \rightarrow \int_{\tilde{s}_{n}} F^{ \pm}\left(x, \widetilde{Q}^{ \pm}(x)\right) d \sigma
$$

as $\beta \downarrow 0$. Now the assertion (9.3) follows from (9.2), (9.6), and (9.7).

9.3. Lemma. Under Assumptions 2.2(a)-(e), let $\widetilde{S}$ denote a weak solution of Problem 2.1, and let $\left(S_{*, n}^{-}, S_{n}, S_{*, n}^{+}\right)_{n=1}^{\infty}$ be an approximating sequence (corresponding to a positive null sequence $\left.\left(\varepsilon_{n}\right)_{n=1}^{\infty}\right)$. For fixed $x_{0} \in \widetilde{S}$, define $\mathcal{L}(x):=\{y=$ $\left.x+t \tilde{\nu}\left(x_{0}\right): t \in \mathbb{R}\right\}$ for all $x \in \widetilde{S}$. Also define $\tilde{s}:=\left\{x \in \widetilde{S}: \tilde{\nu}(x) \cdot \tilde{\nu}\left(x_{0}\right)>\right.$ 
$\left.0, d\left(x, \mathcal{L}\left(x_{0}\right)\right)<\delta\right\}$ for a fixed, sufficiently small value $\delta>0$ (chosen such that $\tilde{\nu}(x) \cdot \tilde{\nu}\left(x_{0}\right)>(1 / 2)$ for all $\left.x \in \tilde{s}\right)$. For each $\alpha \in(0,1)$ and $n \in \mathbb{N}$, define $S_{n, \alpha}^{ \pm}:=\left\{U_{n}^{ \pm}(x)=\alpha \varepsilon_{n}\right\}$ (on which the differential surface area is denoted by $d \sigma_{n, \alpha}^{ \pm}$). Also define $s_{n, \alpha}^{ \pm}:=\phi_{n, \alpha}^{ \pm}(\tilde{s})=\left\{\phi_{n, \alpha}^{ \pm}(x): x \in \tilde{s}\right\}$ for sufficiently large $n \in \mathbb{N}$, where the (invertible) mapping $x_{n, \alpha}^{ \pm}=\phi_{n, \alpha}^{ \pm}(x): \tilde{s} \rightarrow S_{n, \alpha}^{ \pm}$is defined such that $\phi_{n, \alpha}^{ \pm}(x)$ is the point in $S_{n, \alpha}^{ \pm} \cap \mathcal{L}(x)$ which is closest to $x$. Then, for any $\eta>0$, there exists a value $\alpha(\delta, \eta) \in(0,1)$ such that

$$
\int_{s_{n, \alpha}^{-}} F^{-}\left(x, Q_{n}^{-}\left(x_{n, \alpha}^{-}\right)\right) d \sigma_{n, \alpha}^{-}<\int_{s_{n, \alpha}^{+}} F^{+}\left(x, Q_{n}^{+}\left(x_{n, \alpha}^{+}\right)\right) d \sigma_{n, \alpha}^{+}+\eta
$$

whenever $\alpha \in(0, \alpha(\delta, \eta)]$ and $n \in \mathbb{N}$ is sufficiently large (depending on $\alpha$ ).

Proof. Choose local coordinates near the given point $x_{0} \in \widetilde{S}$ such that $x_{0}=0$ and $\tilde{\nu}\left(x_{0}\right)=e_{N}:=(0, \ldots, 0,1)$. In the notation of Lemmas 6.11 and 6.12 , let $z=\widetilde{S}(y): \mathfrak{D}_{\delta} \rightarrow \mathbb{R}$ and $z=S_{n, \alpha}^{ \pm}(y): \mathfrak{D}_{\delta} \rightarrow \mathbb{R}$ be local coordinate representations of the surfaces $\widetilde{S} \cap E_{\delta}$ and $S_{n, \alpha}^{ \pm} \cap E_{\delta}$ (where we write $x=(y, z)=\left(y_{1}, y_{2}, \ldots, y_{N-1}, z\right)$ ). We can define the functions $\mathfrak{F}_{n, \alpha}^{ \pm}(y)=F^{ \pm}\left(y, \widetilde{S}(y), Q_{n}^{ \pm}\left(y, S_{n, \alpha}^{ \pm}(y)\right)\right): \mathfrak{D}_{\delta} \rightarrow \mathbb{R}$ for small $\alpha>0$ and large $n \in \mathbb{N}$. Given $\eta>0$, we have

$$
\mathfrak{F}_{n, \alpha}^{-}(y) \leq \mathfrak{F}_{n, \alpha}^{+}(y)+(\eta / 2|\tilde{s}|)
$$

uniformly in $\mathfrak{D}_{\delta}$ for sufficiently small $\alpha>0$ and for sufficiently large $n \in \mathbb{N}$ (depending on $\alpha$ ), as follows by interpreting Lemma 6.12(a) in the case where $F(x, p, q)=F^{+}(x, q)-F^{-}(x, p)$. We have that

$$
\left.\int_{s_{n, \alpha}^{ \pm}} F^{ \pm}\left(x, Q_{n}^{ \pm}\left(x_{n, \alpha}^{ \pm}\right)\right) d \sigma_{n, \alpha}^{ \pm}=\left.\int_{\mathfrak{D}_{\delta}} \mathfrak{F}_{n, \alpha}^{ \pm}(y)\left(\mid \nabla S_{n, \alpha}^{ \pm}(y)\right)\right|^{2}+1\right)^{1 / 2} d y
$$

for small $\alpha>0$ and large $n \in \mathbb{N}$. The assertion now follows from (9.9) and (9.10), in view of the fact that, for fixed sufficiently small $\alpha>0$, the functions $Q_{n}^{ \pm}\left(y, S_{n, \alpha}^{ \pm}(y)\right): \mathfrak{D}_{\delta} \rightarrow \mathbb{R}$ are uniformly bounded and uniformly positive as $n \rightarrow \infty$ (by Lemmas 4.8 and $6.12(\mathrm{~b})$ ), and the fact that $\left|\nabla S_{n, \alpha}^{ \pm}(y)-\nabla \widetilde{S}(y)\right| \rightarrow 0$ uniformly in $\mathfrak{D}_{\delta}$ as $n \rightarrow \infty$ (see Lemmas 6.5 and 6.11).

9.4. Lemma. In the context of Lemma 9.3, let the values $\delta, \eta, \alpha>0$ be given such that $\delta$ is sufficiently small and $\alpha \in(0, \alpha(\delta, \eta)$ ] (so that (9.8) holds for sufficiently large $n \in \mathbb{N})$. For any $\beta \in(0,1)$, and for sufficiently large $n \in \mathbb{N}$, let each point $x \in \tilde{s}$ correspond to the points $x_{n, \alpha}^{ \pm}=\phi_{n, \alpha}^{ \pm}(x) \in S_{n, \alpha}^{ \pm}$and $\hat{x}_{n, \alpha, \beta}^{ \pm}=\hat{\psi}_{n, \alpha, \beta}^{ \pm}\left(x_{n, \alpha}^{ \pm}\right)=$ $\hat{\psi}_{n, \alpha, \beta}^{ \pm}\left(\phi_{n, \alpha}^{ \pm}(x)\right) \in \widehat{S}_{n, \beta}^{ \pm}:=\left\{U_{n}^{ \pm}(x)=\beta\right\}$, where the (invertible) function $\hat{\psi}_{n, \alpha, \beta}^{ \pm}(y)$ : $S_{n, \alpha}^{ \pm} \rightarrow \widehat{S}_{n, \beta}^{ \pm}$is uniquely defined such that $y \in S_{n, \alpha}^{ \pm}$and $\hat{\psi}_{n, \alpha, \beta}^{ \pm}(y) \in \widehat{S}_{n, \beta}^{ \pm}$are located on the same curve of steepest ascent of the function $U_{n}^{ \pm}(\cdot)$. Let $\hat{s}_{n, \alpha, \beta}^{ \pm}=\left\{\hat{\psi}_{n, \alpha, \beta}^{ \pm}(y)\right.$ : $\left.y \in s_{n, \alpha}^{ \pm}\right\} \subset \widehat{S}_{n, \beta}^{ \pm}$for any $\beta \in(0,1)$ and sufficiently large $n \in \mathbb{N}$. Then

$$
\int_{\hat{s}_{n, \alpha, \beta}^{-}} F^{-}\left(x, Q_{n}^{-}\left(\hat{x}_{n, \alpha, \beta}^{-}\right)\right) d \hat{\sigma}_{n, \beta}^{-}<\int_{\hat{s}_{n, \alpha, \beta}^{+}} F^{+}\left(x, Q_{n}^{+}\left(\hat{x}_{n, \alpha, \beta}^{+}\right)\right) d \hat{\sigma}_{n, \beta}^{+}+2 \eta
$$

provided that $\beta$ is sufficiently small and $n \in \mathbb{N}$ is sufficiently large (depending on $\delta, \eta, \alpha, \beta)$, where $\hat{\sigma}_{n, \beta}^{ \pm}$denotes the differential area of $\widehat{S}_{n, \beta}^{ \pm}$. 
Proof. We remark that the differential areas of the surfaces $S_{n, \alpha}^{ \pm}$and $\widehat{S}_{n, \beta}^{ \pm}$are related by $Q_{n}^{ \pm}\left(x_{n, \alpha}^{ \pm}\right) d \sigma_{n, \alpha}^{ \pm}=Q_{n}^{ \pm}\left(\hat{x}_{n, \alpha, \beta}^{ \pm}\right) d \hat{\sigma}_{n, \beta}^{ \pm}$. For fixed $\alpha \in(0, \alpha(\delta, \eta)]$ and $\beta \in(0,1)$, it follows from Lemma 4.8 and Lemma 6.12(b) that

$$
\begin{aligned}
& 0<\mathfrak{C}_{0}<Q_{n}^{+}\left(\hat{x}_{n, \alpha, \beta}^{+}\right) \leq Q_{n}^{+}\left(x_{n, \alpha}^{+}\right) \leq \mathfrak{C}^{+}(\alpha), \\
& 0<\mathfrak{C}^{-}(\alpha) \leq Q_{n}^{-}\left(x_{n, \alpha}^{-}\right) \leq Q_{n}^{-}\left(\hat{x}_{n, \alpha, \beta}^{-}\right) \leq \mathfrak{C}_{1},
\end{aligned}
$$

provided that $n \in \mathbb{N}$ is sufficiently large (so that $S_{n, \alpha}^{+}<\hat{S}_{\beta}^{+}$and $S_{n, \alpha}^{-}>\widehat{S}_{\beta}^{-}$). It directly follows from (9.12) and (9.13) that

$$
\begin{aligned}
& \pm\left(\int_{\hat{s}_{n, \alpha, \beta}^{ \pm}} F^{ \pm}\left(x, Q_{n}^{ \pm}\left(\hat{x}_{n, \alpha, \beta}^{ \pm}\right)\right) d \hat{\sigma}_{n, \beta}^{ \pm}-\int_{s_{n, \alpha}^{ \pm}} F^{ \pm}\left(x, Q_{n}^{ \pm}\left(x_{n, \alpha}^{ \pm}\right)\right) d \sigma_{n, \alpha}^{ \pm}\right) \\
& \quad= \pm \int_{s_{n, \alpha}^{ \pm}}\left(F^{ \pm}\left(x, Q_{n}^{ \pm}\left(\hat{x}_{n, \alpha, \beta}^{ \pm}\right)\right)\left(Q_{n}^{ \pm}\left(x_{n, \alpha}^{ \pm}\right) / Q_{n}^{ \pm}\left(\hat{x}_{n, \alpha, \beta}^{ \pm}\right)\right)-F^{ \pm}\left(x, Q^{ \pm}\left(x_{n, \alpha}^{ \pm}\right)\right)\right) d \sigma_{n, \alpha}^{ \pm} \\
& \quad \geq \pm \int_{s_{n, \alpha}^{ \pm}}\left(F^{ \pm}\left(x, Q_{n}^{ \pm}\left(\hat{x}_{n, \alpha, \beta}^{ \pm}\right)\right)-F^{ \pm}\left(x, Q_{n}^{ \pm}\left(x_{n, \alpha}^{ \pm}\right)\right)\right) d \sigma_{n, \alpha}^{ \pm} \\
& \quad \geq \pm L \int_{s_{n, \alpha}^{ \pm}}\left(Q_{n}^{ \pm}\left(\hat{x}_{n, \alpha, \beta}^{ \pm}\right)-Q_{n}^{ \pm}\left(x_{n, \alpha}^{ \pm}\right)\right) d \sigma_{n, \alpha}^{ \pm} \\
& \quad \geq \pm L \mathfrak{C}^{ \pm}(\alpha) \int_{s_{n, \alpha}^{ \pm}}\left(\left[Q_{n}^{ \pm}\left(\hat{x}_{n, \alpha, \beta}^{ \pm}\right)-Q_{n}^{ \pm}\left(x_{n, \alpha}^{ \pm}\right)\right] / Q_{n}^{ \pm}\left(\hat{x}_{n, \alpha, \beta}^{ \pm}\right)\right) d \sigma_{n, \alpha}^{ \pm},
\end{aligned}
$$

where $L$ is a constant such that $\partial F^{+}(x, t) / \partial t \leq L$ for $t \in\left[\mathfrak{C}_{0}, \mathfrak{C}^{+}(\alpha)\right]$ and $\partial F^{-}(x, t) / \partial t \leq L$ for $t \in\left[\mathfrak{C}^{-}(\alpha), \mathfrak{C}_{1}\right]$ (both for all $\left.x \in \Omega_{*}\right)$. On the other hand,

$$
\int_{s_{n, \alpha}^{ \pm}}\left(\left(Q_{n}^{ \pm}\left(x_{n, \alpha}^{ \pm}\right) / Q_{n}^{ \pm}\left(\hat{x}_{n, \alpha, \beta}^{ \pm}\right)\right)-1\right) d \sigma_{n, \alpha}^{ \pm}=\left(\left|\hat{s}_{n, \alpha, \beta}^{ \pm}\right|-\left|s_{n, \alpha}^{ \pm}\right|\right) \rightarrow 0
$$

as $\beta \downarrow 0$ and $n \rightarrow \infty$, due to the fact that $\left|s_{n, \alpha}^{ \pm}\right| \rightarrow|\tilde{s}|$ and $\left|\hat{s}_{n, \alpha, \beta}^{ \pm}\right| \rightarrow|\tilde{s}|$, both as $\beta \downarrow 0$ and $n \rightarrow \infty$ (by Proposition 7.2 , where $|\cdot|$ denotes $(N-1)$-dimensional surface area). The assertion follows from (9.14), (9.15), and Lemma 9.3 (eq. (9.8)).

9.5. Lemma. Under Assumptions 2.2(a)-(e), let $\widetilde{S}$ denote a weak solution of Problem 2.1, and let $\left(S_{*, n}^{-}, S_{n}, S_{*, n}^{+}\right)_{n=1}^{\infty}$ be a corresponding approximating sequence. For fixed $x_{0} \in \widetilde{S}$, let $\tilde{s}:=\left\{x \in \widetilde{S}: \tilde{\nu}(x) \cdot \tilde{\nu}\left(x_{0}\right)>0, d\left(x, \mathcal{L}\left(x_{0}\right)\right)<\delta\right\}$ (where $\left.\mathcal{L}\left(x_{0}\right)=\left\{x_{0}+\tilde{\nu}\left(x_{0}\right) t: t \in \mathbb{R}\right\}\right)$ for a fixed, sufficiently small value $\delta>0$. For any $\beta \in(0,1)$, let $\widetilde{S}_{\beta}^{ \pm}:=\left\{\widetilde{U}^{ \pm}(x)=\beta\right\}$, and let the function $x_{\beta}^{ \pm}=\tilde{\psi}_{\beta}^{ \pm}(x): \widetilde{S} \rightarrow \widetilde{S}_{\beta}^{ \pm}$be defined such that $x$ and $x_{\beta}^{ \pm}$always lie on the same curve of steepest ascent of the function $\widetilde{U}^{ \pm}(\cdot)$. For any $\alpha, \beta \in(0,1), x \in \tilde{s}$, and sufficiently large $n \in \mathbb{N}$, let the corresponding point $\hat{x}_{n, \alpha, \beta}^{ \pm}:=\hat{\psi}_{n, \alpha, \beta}^{ \pm}\left(\phi_{n, \alpha}^{ \pm}(x)\right) \in \widehat{S}_{n, \beta}^{ \pm}:=\left\{U_{n}^{ \pm}(x)=\beta\right\}$ be as defined in Lemmas 9.3 and 9.4. Finally, define the subsurfaces $\tilde{s}_{\beta}^{ \pm}=\left\{x_{\beta}^{ \pm}: x \in \tilde{s}\right\} \subset \widetilde{S}_{\beta}^{ \pm}$ and $\hat{s}_{n, \alpha, \beta}^{ \pm}=\left\{\hat{x}_{n, \alpha, \beta}^{ \pm}: x \in \tilde{s}\right\} \subset \widehat{S}_{n, \beta}^{ \pm}$(with $(N-1)$-dimensional differential surface areas denoted respectively by $d \sigma_{\beta}^{ \pm}$and $\left.d \hat{\sigma}_{n, \beta}^{ \pm}\right)$. Then for any fixed $\alpha, \beta \in(0,1)$ :

$$
\lim _{n \rightarrow \infty} \int_{\hat{s}_{n, \alpha, \beta}^{ \pm}} F^{ \pm}\left(x, Q_{n}^{ \pm}\left(\hat{x}_{n, \alpha, \beta}^{ \pm}\right)\right) d \hat{\sigma}_{n, \beta}^{ \pm}=\int_{\tilde{s}_{\beta}^{ \pm}} F^{ \pm}\left(x, \widetilde{Q}^{ \pm}\left(x_{\beta}^{ \pm}\right)\right) d \sigma_{\beta}^{ \pm} .
$$


Proof. Define the mapping $g_{\beta}^{ \pm}(x): \widetilde{S}_{\beta}^{ \pm} \rightarrow \widetilde{S}$ such that $x \in \widetilde{S}_{\beta}^{ \pm}$and $g_{\beta}^{ \pm}(x) \in \widetilde{S}$ always lie on the same curve of steepest ascent of the function $\widetilde{U}^{ \pm}$(thus $\tilde{\psi}_{\beta}^{ \pm}\left(g_{\beta}^{ \pm}(x)\right)=x$ in $\widetilde{S}_{\beta}^{ \pm}$). For each sufficiently large $n \in \mathbb{N}$ (so that $\tilde{\nu}(x) \cdot \nu_{n}(x) \geq 1 / 2$ throughout $K \backslash S_{n}$, where $K$ is a fixed, sufficiently large subset of $\Omega_{*}$ ), we define the mapping $E_{n, \beta}^{ \pm}(x): \widetilde{S}_{\beta}^{ \pm} \rightarrow \widehat{S}_{n, \beta}^{ \pm}$such that $x \in \widetilde{S}_{\beta}^{ \pm}$and $E_{n, \beta}^{ \pm}(x) \in \widehat{S}_{n, \beta}^{ \pm}$always lie on the same curve of steepest ascent of the function $U_{n}^{ \pm}$. Let $g_{n, \alpha, \beta}^{ \pm}(x): \hat{s}_{n, \alpha, \beta}^{ \pm} \rightarrow \tilde{s}$ denote the inverse of the composite mapping $\hat{\psi}_{n, \alpha, \beta}^{ \pm}\left(\phi_{n, \alpha}^{ \pm}(x)\right): \tilde{s} \rightarrow \hat{s}_{n, \alpha, \beta}^{ \pm}$defined in Lemmas 9.3 and 9.4 . By a change of variables, we have

$$
\begin{aligned}
& \int_{\tilde{s}_{\beta}^{ \pm}} F^{ \pm}\left(x, \widetilde{Q}^{ \pm}\left(x_{\beta}^{ \pm}\right)\right) d \sigma_{\beta}^{ \pm}=\int_{\tilde{s}_{\beta}^{ \pm}} F^{ \pm}\left(g_{\beta}^{ \pm}(x), \widetilde{Q}^{ \pm}(x)\right) d \sigma_{\beta}^{ \pm}, \\
& \int_{\hat{s}_{n, \alpha, \beta}^{ \pm}} F^{ \pm}\left(x, Q_{n}^{ \pm}\left(\hat{x}_{n, \alpha, \beta}^{ \pm}\right)\right) d \hat{\sigma}_{n, \beta}^{ \pm} \\
& \quad=\int_{\tilde{s}_{n, \alpha, \beta}^{ \pm}} F^{ \pm}\left(g_{n, \alpha, \beta}^{ \pm}\left(E_{n, \beta}^{ \pm}(x)\right), Q_{n}^{ \pm}\left(E_{n, \beta}^{ \pm}(x)\right)\right) R_{n, \beta}^{ \pm}(x) d \sigma_{\beta}^{ \pm},
\end{aligned}
$$

where the subsurface $\tilde{s}_{n, \alpha, \beta}^{ \pm} \subset \widetilde{S}_{\beta}^{ \pm}$is chosen such that $E_{n, \beta}^{ \pm}\left(\tilde{s}_{n, \alpha, \beta}^{ \pm}\right)=\hat{s}_{n, \alpha, \beta}^{ \pm}$, and where we define

$$
R_{n, \beta}^{ \pm}(x)=\left(\nabla U_{n}^{ \pm}(x) \cdot \nabla \widetilde{U}^{ \pm}(x) / \widetilde{Q}^{ \pm}(x) Q_{n}^{ \pm}\left(E_{n, \beta}^{ \pm}(x)\right)\right)
$$

for all $x \in \widetilde{S}_{\beta}^{ \pm}$. Here, the left-hand sides of (9.17) and (9.18) are based on the notation introduced in the statement of Lemma 9.5, whereas we assume that $x \in \widetilde{S}_{\beta}^{ \pm}$ in the right-hand sides of these equations. Now $E_{n, \beta}^{ \pm}(x) \rightarrow x$ as $n \rightarrow \infty$, uniformly over $x \in S_{\beta}^{ \pm}$, due to Lemma 6.5 (or Lemma 7.3). Since $\nabla U_{n}^{ \pm}(x) \rightarrow \nabla \widetilde{U}(x)$ as $n \rightarrow \infty$, uniformly in compact subsets of $\widetilde{\Omega}^{ \pm}$, it is clear that $Q_{n}^{ \pm}\left(E_{n, \beta}^{ \pm}(x)\right) \rightarrow \widetilde{Q}^{ \pm}(x)$ and $R_{n, \beta}^{ \pm}(x) \rightarrow 1$ (both uniformly relative to $x \in \widetilde{S}_{\beta}^{ \pm}$) as $n \rightarrow \infty$. Moreover, Lemma 7.3 implies that $g_{n, \alpha, \beta}^{ \pm}\left(y_{n}\right) \rightarrow g_{\beta}^{ \pm}(x)$ as $n \rightarrow \infty$ for any sequence $\left(y_{n}\right)_{n=1}^{\infty}$ such that $y_{n} \in \hat{s}_{n, \alpha, \beta}^{ \pm}$for each $n \in \mathbb{N}$, and such that $y_{n} \rightarrow x \in \tilde{s}_{\beta}^{ \pm}$as $n \rightarrow \infty$, and it follows from this that $g_{n, \alpha, \beta}^{ \pm}\left(E_{n, \beta}^{ \pm}(x)\right) \rightarrow g_{\beta}^{ \pm}(x)$ as $n \rightarrow \infty$ for any fixed $x \in \tilde{s}_{\beta}^{ \pm}$. Finally, it follows from Proposition 7.2 that $\left|\tilde{s}_{n, \alpha, \beta}^{ \pm} \backslash \tilde{s}_{\beta}^{ \pm}\right| \rightarrow 0$ and $\left|\tilde{s}_{\beta}^{ \pm} \backslash \tilde{s}_{n, \alpha, \beta}^{ \pm}\right| \rightarrow 0$ both as $n \rightarrow \infty$. In view of the assumed continuity of the functions $F^{ \pm}(x, t)$, it easily follows that the right-hand side of (9.18) converges to the right-hand side of (9.17) as $n \rightarrow \infty$.

9.6. Proof of Theorem 9.1. Let $\widetilde{S}$ be a weak solution, and $\left(S_{*, n}^{-}, S_{n}, S_{*, n}^{+}\right)_{n=1}^{\infty}$ be an approximating sequence. Suppose that $F^{-}\left(x_{0}, \widetilde{Q}^{-}\left(x_{0}\right)\right)>F^{+}\left(x_{0}, \widetilde{Q}^{+}\left(x_{0}\right)\right)$ at a point $x_{0} \in \widetilde{S}$. Then, by Lemma 9.2 (eq. (9.3)), there exist values $\eta_{0}>0, \delta>0$, and $\beta_{0} \in(0,1)$ such that $\tilde{\nu}(x) \cdot \tilde{\nu}\left(x_{0}\right)>(1 / 2)$ for all $x \in \tilde{s}$, and such that

$$
\int_{\tilde{s}_{\beta}^{+}} F^{+}\left(x, \widetilde{Q}^{+}\left(x_{\beta}^{+}\right)\right) d \sigma_{\beta}^{+}<\int_{\tilde{s}_{\beta}^{-}} F^{-}\left(x, \widetilde{Q}^{-}\left(x_{\beta}^{-}\right)\right) d \sigma_{\beta}^{-}-\eta_{0}
$$

for all $\beta \in\left(0, \beta_{0}\right]$, where $\tilde{s}:=\left\{x \in \widetilde{S}: \tilde{\nu}(x) \cdot \tilde{\nu}\left(x_{0}\right)>0, d\left(x, \mathcal{L}\left(x_{0}\right)\right)<\delta\right\}, \mathcal{L}\left(x_{0}\right)=$ $\left\{x_{0}+\tilde{\nu}\left(x_{0}\right) t: t \in \mathbb{R}\right\}$, and $\tilde{s}_{\beta}^{ \pm}$denotes the projection of $\tilde{s}$ on $\widetilde{S}_{\beta}^{ \pm}$along the curves of 
steepest ascent of $\widetilde{U}^{ \pm}(x)$. However, for the same fixed value $\delta>0$, it follows from Lemma 9.4 (in the notation given there) that

$$
\int_{\hat{s}_{n, \alpha, \beta}^{+}} F^{+}\left(x, Q_{n}^{+}\left(\hat{x}_{n, \alpha, \beta}^{+}\right)\right) d \hat{\sigma}_{n, \beta}^{+} \geq \int_{\hat{s}_{n, \alpha, \beta}^{-}} F^{-}\left(x, Q_{n}^{-}\left(\hat{x}_{n, \alpha, \beta}^{-}\right)\right) d \hat{\sigma}_{n, \beta}^{-}-\left(\eta_{0} / 2\right)
$$

for any sufficiently small $\alpha, \beta \in(0,1)$, provided the $n \in \mathbb{N}$ is sufficiently large (depending on $\alpha, \beta, \eta_{0}$ ). Due to Lemma 9.5, it follows from (9.19) in the limit as $n \rightarrow \infty$ that

$$
\int_{\tilde{s}_{\beta}^{+}} F^{+}\left(x, \widetilde{Q}^{+}\left(x_{\beta}^{+}\right)\right) d \sigma_{\beta}^{+} \geq \int_{\tilde{s}_{\beta}^{-}} F^{-}\left(x, \widetilde{Q}^{-}\left(x_{\beta}^{-}\right)\right) d \sigma_{\beta}^{-}-\left(\eta_{0} / 2\right)
$$

for any sufficiently small value $\beta \in(0,1)$. This contradiction proves the assertion.

\section{Proof of Theorem 2.3(B)}

10.1. Theorem. Let $\widetilde{S}$ denote a (convex) weak solution of Problem 2.1, where Assumptions 2.2(a)-(e) apply. Then: (a) The functions $\nabla \widetilde{U}^{ \pm}(x): \widetilde{\Omega}^{ \pm} \rightarrow \mathbb{R}$ have continuous, nonvanishing continuations to $\widetilde{\Omega}^{ \pm} \cup \widetilde{S}$. (b) We have

$$
F^{-}\left(x_{0}, \widetilde{Q}^{-}\left(x_{0}\right)\right)=F^{+}\left(x_{0}, \widetilde{Q}^{+}\left(x_{0}\right)\right)
$$

at all points $x_{0} \in \widetilde{S}$, where $\widetilde{Q}^{ \pm}(x):=\left|\nabla \widetilde{U}^{ \pm}(x)\right|$ throughout $\widetilde{\Omega}^{ \pm} \cup \widetilde{S}$.

Proof. By Theorems 7.1 and 9.1, eq. (10.1) holds at all points $x_{0} \in \widetilde{S}$, where $\widetilde{Q}^{ \pm}\left(x_{0}\right)$ is defined on $\widetilde{S}$ by Theorem $6.2(\mathrm{c})$. Since the functions $\pm \widetilde{Q}^{ \pm}\left(x_{0}\right): \widetilde{S} \rightarrow \mathbb{R}$ are both lower semicontinuous (by Theorem 6.2(d)), it follows from the assumed properties of the functions $F^{ \pm}(x, t)$ that the functions $\pm F^{ \pm}\left(x_{0}, \widetilde{Q}^{ \pm}\left(x_{0}\right)\right): \widetilde{S} \rightarrow \mathbb{R}$ are also both lower semicontinuous. In view of (10.1), it follows that both functions are actually continuous on $\widetilde{S}$. Since the mappings $\tau=F^{ \pm}(x, t)$ have continuous inverses of the form $t=G^{ \pm}(x, \tau)$, it follows that the functions $\widetilde{Q}^{ \pm}\left(x_{0}\right): \widetilde{S} \rightarrow \mathbb{R}$ are also continuous. For $\varepsilon \in(0,1)$, let $\pi_{\varepsilon}^{ \pm}: \widetilde{S} \rightarrow \Phi_{\varepsilon}^{ \pm}\left(\widetilde{S}, S_{*}^{ \pm}\right)$denote the continuous mapping such that $x_{0} \in \widetilde{S}$ and $\pi_{\varepsilon}^{ \pm}\left(x_{0}\right)$ always lie on the same curve of steepest ascent of $\widetilde{U}^{ \pm}(\cdot)$ (see Lemma 7.3 and [9], Lemma 4.4). For each $x_{0} \in \widetilde{S}$ and $\varepsilon \in(0,1)$, define $\phi_{\varepsilon}^{ \pm}\left(x_{0}\right)=\widetilde{Q}^{ \pm}\left(\pi_{\varepsilon}^{ \pm}\left(x_{0}\right)\right)$. Then the continuous functions $\phi_{\varepsilon}^{ \pm}\left(x_{0}\right): \widetilde{S} \rightarrow \mathbb{R}$ are such that $\phi_{\varepsilon}^{+}\left(x_{0}\right) \uparrow \widetilde{Q}^{+}\left(x_{0}\right)$ and $\phi_{\varepsilon}^{-}\left(x_{0}\right) \downarrow \widetilde{Q}^{-}\left(x_{0}\right)$ pointwise as $\varepsilon \downarrow 0$, by Theorem 6.2(f). Therefore, the convergence is uniform, due to the continuity of the functions $\widetilde{Q}^{ \pm}\left(x_{0}\right): \widetilde{S} \rightarrow \mathbb{R}$. Therefore, there exists a null function $z(\cdot)$ such that $\left|\widetilde{Q}^{ \pm}(x)-\widetilde{Q}^{ \pm}(p(x))\right| \leq z(d(x, \widetilde{S}))$ for all $x \in \widetilde{\Omega}^{ \pm}$, where $p(x) \in \widetilde{S}$ denotes the endpoint of the arc of steepest descent of $\widetilde{U}^{ \pm}$joining $x$ to $\widetilde{S}$. Also, there exists a null function $z(\cdot)$ such that for any points $x_{0} \in \widetilde{S}$ and $x \in \widetilde{\Omega}^{ \pm}$, we have

$$
|x-p(x)| \leq\left(\widetilde{U}^{ \pm}(x) / \mathfrak{C}_{0}\right) \leq z(d(x, \widetilde{S})) \leq z\left(\left|x-x_{0}\right|\right)
$$

and therefore

$$
\left|p(x)-x_{0}\right| \leq\left|x-x_{0}\right|+|p(x)-x| \leq\left(\left|x-x_{0}\right|+z\left(\left|x-x_{0}\right|\right)\right) .
$$

In view of these facts, it follows directly from the triangle inequality, in the form:

$$
\left|\widetilde{Q}^{ \pm}(x)-\widetilde{Q}^{ \pm}\left(x_{0}\right)\right| \leq\left|\widetilde{Q}^{ \pm}(x)-\widetilde{Q}^{ \pm}(p(x))\right|+\left|\widetilde{Q}^{ \pm}(p(x))-\widetilde{Q}^{ \pm}\left(x_{0}\right)\right|
$$


that $\widetilde{Q}^{ \pm}(x) \rightarrow \widetilde{Q}^{ \pm}\left(x_{0}\right)$ as $x \rightarrow x_{0} \in \widetilde{S}$ (for $x \in \widetilde{\Omega}^{ \pm}$). The continuity of the functions $\nabla \widetilde{U}^{ \pm}(x): \widetilde{S} \cup \widetilde{\Omega}^{ \pm} \rightarrow \mathbb{R}$ (defined on $\widetilde{S}$ by Theorem 6.2(c)) now follows from Lemma 6.4 .

\section{Appendix: Remarks on Example 2.9}

The purpose of this appendix is to outline the demonstration that, under the stated assumptions, the function $F(x, p, q)$ introduced in Example 2.9 satisfies Assumption 2.2(d). As in Remarks 2.5, we define the function $h(x, q)=$ $\left(A+B q^{-\beta}\right)^{-(1 / \alpha)}$. Rather than introduce a linear function $\phi(\lambda):[0,1] \rightarrow \mathbb{R}^{N}$ as suggested there, we simply assume that $x \in \mathbb{R}$. The condition $h_{q q} \leq 0$ reduces to

$$
((\alpha+1) \beta / \alpha(\beta+1)) B \leq A q^{\beta}+B
$$

which is satisfied if

$$
\beta<\alpha .
$$

By grouping the coefficients of distinct functions of $q$, the condition $h_{x x} \leq 0$ is seen to be satisfied if

$$
\begin{gathered}
((\alpha+1) / \alpha) A_{x}^{2} \leq A A_{x x} ; \quad((\alpha+1) / \alpha) B_{x}^{2} \leq B B_{x x} \\
2((\alpha+1) / \alpha) A_{x} B_{x} \leq A B_{x x}+B A_{x x} .
\end{gathered}
$$

We assume $(\mathrm{A} 2 \mathrm{a}, \mathrm{b})$, and observe that $(\mathrm{A} 2 \mathrm{c})$ follows from them in view of the inequality

$$
((\alpha+1) / \alpha)\left|A_{x}\right|\left|B_{x}\right| \leq\left(A B_{x x} B A_{x x}\right)^{1 / 2} \leq(1 / 2)\left(A B_{x x}+B A_{x x}\right) .
$$

Finally, by again grouping the coefficients of distinct functions of $q$ (and still assuming (A1) and $(\mathrm{A} 2 \mathrm{a}, \mathrm{b}))$, one sees that the requirement $h_{x q}^{2} \leq h_{x x} h_{q q}$ will be satisfied if

$$
\begin{gathered}
(1+(1 /(\alpha-\beta))) B_{x}^{2} \leq B B_{x x}, \\
2(\alpha+1) B A_{x} B_{x}+\alpha \beta A B_{x}^{2} \leq \alpha(\beta+1) A B B_{x x}+(\alpha-\beta) B^{2} A_{x x} .
\end{gathered}
$$

We assume (A3) (which implies (A2b)). In view of (A3), the requirement (A4) will be satisfied if

$$
2 A_{x} B_{x} \leq(\alpha /(\alpha+1-\beta)) A B_{x x}+((\alpha-\beta) /(\alpha+1)) B A_{x x} .
$$

However, it follows from (A2a) and (A3) that

$$
2\left|A_{x}\right|\left|B_{x}\right| \leq 2\left(\lambda A B_{x x} \mu B A_{x x}\right)^{1 / 2} \leq \lambda A B_{x x}+\mu B A_{x x}
$$

where we set $\lambda=(\alpha /(\alpha+1-\beta))$ and $\mu=((\alpha-\beta) /(\alpha+1))$. This completes the proof.

\section{REFERENCES}

1. A. Acker, Free-boundary optimization - a constructive, iterative method, J. Appl. Math. Phys. (ZAMP) 30 (1979), 885-900. MR 81k:31006

2. - Interior free boundary problems for the Laplace equation, Arch. Rat'l. Mech. Anal. 75 (1981), 157-168. MR 82f:35185

3. - On the convexity of equilibrium plasma configurations, Math. Meth. Appl. Sci. 3 (1981), 435-443. MR 83g:76106 
4. - How to approximate the solutions for certain free boundary problems for the Laplace equation by using the contraction principle, Z. Angew. Math. Phys. (ZAMP) 32 (1981), 22-33. MR 82m:35004

5. _ On the convexity and the successive approximation of solutions in a free boundary problem with two fluid phases, Comm. Part. Diff. Eqs. 14 (1989), 1635-1652. MR 91d:35235

6. - On the nonconvexity of solutions in free boundary problems arising in plasma physics and fluid dynamics, Comm. Pure Appl. Math. 42 (1989), 1165-1174 (Addendum: 44 (1991), 869-872). MR 91a:35167; MR 92h:35243

7. _ , A multilayer problem. In : Free Boundary Problems in Fluid Flow with Applications (Proceedings of the International Conference on Free Boundary Problems, Montreal, 1990), pp. 44-52. Pitman Res. Notes in Math. Ser., \# 282. Longman Sci. Tech., Harlow, 1993. MR 94g:35222

8. _ On the multilayer fluid problem: regularity, uniqueness, convexity, and successive approximation of solutions, Comm. Part. Diff. Eqs. 16 (1991), 647-666. MR 92k:35290

9. _ On the existence of convex classical solutions to multilayer fluid problems in arbitrary space dimensions, Pacific J. Math. 162 (1994), 201-231. MR 94j:35196

10. _ Convex free boundaries and the operator method, in Variational Problems (A. Friedman and J. Spruck, Editors), IMA Volumes in Mathematics and its Applications \# 53, Springer-Verlag, 1993. MR 96a:35222

11. __ On 2-layer free boundary problems with generalized joining conditions: convexity and successive approximation of solutions. In: Comparison Methods and Stability Theory, edited by Xinzhi Liu and David Siegel. Lecture Notes in Pure and Applied Mathematics, Vol. 162, Marcel Dekker, Inc., New York, 1994. MR 95e:35229

12. A. Acker and R. Meyer, A free boundary problem for the p-laplacian: uniqueness, convexity, and successive approximation of solutions, Electronic J. Diff. Eqs. 1995, No. 8, approx. 20 pp. MR 96c:35198

13. H. W. Alt, L. A. Caffarelli and A. Friedman, Variational problems with two fluid phases and their free boundaries, Trans. Amer. Math. Soc. 282 (1984), 431-461. MR 85h:49014

14. L. A. Caffarelli and A. Friedman, Convexity of solutions of semilinear elliptic equations, Duke Math. J. 52 (1985), 431-456. MR 87a:35028

15. L. A. Caffarelli and J. Spruck, Convexity properties of some classical variational problems, Comm. Part. Diff. Eqs. 7 (1982), 1337-1379. MR 85f:49062

16. A. Friedman, Variational principles and free boundary problems, Wiley, 1982. MR 84e:35153

17. R. Gabriel, A result concerning convex level surfaces of 3-dimensional harmonic functions, J. London Math. Soc. 32 (1957), 286-294. MR 19:848a

18. D. Gilbarg and N. Trudinger, Elliptic Partial Differential Equations of Second Order (2nd ed.), Springer-Verlag, 1983. MR 86c:35035

19. N. Korevaar and J. Lewis, Convex solutions to certain elliptic equations have constant rank Hessians, Arch. Rat'l. Mech. Anal. 97 (1987), 19-32. MR 88i:35054

20. P. Laurence and E. Stredulinsky, A new approach to queer differential equations, Comm. Pure Appl. Math. 38 (1985), 333-355. MR 87f:35246

21. __ Existence of regular solutions with convex level sets for semilinear elliptic equations with nonmonotone $L^{1}$ nonlinearities, Part I: an approximating free boundary problem, Indiana U. Math. J. 39 (1990), 1081-1114. MR 92m:35279

22. J. L. Lewis, Capacitary functions in convex rings, Arch. Rat'l. Mech. Anal. 66 (1977), 201224. MR 57:16638

23. R. Meyer, Approximation of the solutions of free boundary problems for the p-Laplace equation, Doctoral dissertation, Wichita State University, May, 1993.

Department of Mathematics and Statistics, Wichita State University, Wichita, KANSAS 67260-0033 